\title{
YOUNG STELLAR OBJECTS IN LYNDS 1641: DISKS, ACCRETION, AND STAR FORMATION HISTORY
}

\author{
Min Fang ${ }^{1,2,3}$, Jinyoung Serena Kim ${ }^{4}$, Roy van Boekel ${ }^{2}$, Aurora Sicilia-Aguilar ${ }^{5}$, \\ Thomas HenNing ${ }^{2}$, and KeVIn Flaherty ${ }^{4}$ \\ ${ }^{1}$ Purple Mountain Observatory and Key Laboratory of Radio Astronomy, Chinese Academy of Sciences, 2 West Beijing Road, \\ 210008 Nanjing, China; mfang@pmo.ac.cn \\ ${ }^{2}$ Max-Planck Institute for Astronomy, Königstuhl 17, D-69117 Heidelberg, Germany \\ ${ }^{3}$ Key Laboratory of Modern Astronomy and Astrophysics (Nanjing University), Ministry of Education, Nanjing 210093, China \\ ${ }^{4}$ Steward Observatory, University of Arizona, 933 North Cherry Avenue, Tucson, AZ 85721-0065, USA \\ ${ }^{5}$ Departamento de Física Teórica, Facultad de Ciencias, Universidad Autonóma de Madrid, 28049 Cantoblanco, Madrid, Spain \\ Received 2012 May 10; accepted 2013 April 26; published 2013 June 14
}

\begin{abstract}
We investigate the young stellar objects (YSOs) in the Lynds 1641 (L1641) cloud using multi-wavelength data including Spitzer, WISE, the Two Micron All Sky Survey, and XMM covering $~ 1390$ YSOs across a range of evolutionary stages. In addition, we targeted a sub-sample of YSOs for optical spectroscopy with the MMT/Hectospec and the MMT/Hectochelle. We use these data, along with archival photometric data, to derive spectral types, extinction values, masses, ages, and accretion rates. We obtain a disk fraction of $\sim 50 \%$ in L1641. The disk frequency is almost constant as a function of stellar mass with a slight peak at $\log \left(M_{*} / M_{\odot}\right) \approx-0.25$. The analysis of multi-epoch spectroscopic data indicates that the accretion variability of YSOs cannot explain the two orders of magnitude of scatter for YSOs with similar masses. Forty-six new transition disk (TD) objects are confirmed in this work, and we find that the fraction of accreting TDs is lower than for optically thick disks (40\%-45\% versus $77 \%-79 \%$, respectively). We confirm our previous result that the accreting TDs have a median accretion rate similar to normal optically thick disks. We confirm that two star formation modes (isolated versus clustered) exist in L1641. We find that the diskless YSOs are statistically older than the YSOs with optically thick disks and the TD objects have a median age that is intermediate between those of the other two populations. We tentatively study the star formation history in L1641 based on the age distribution and find that star formation started to be active 2-3 Myr ago.
\end{abstract}

Key words: accretion, accretion disks - protoplanetary disks - stars: pre-main sequence

Online-only material: color figures, machine-readable tables

\section{INTRODUCTION}

Circumstellar disks, as a by-product of the star formation process via angular momentum conservation, play a key role in the formation of new stars and subsequent planetary systems. An investigation of the disk evolution is thus important in order to understand both star formation and planet formation.

Disk dissipation processes have been constrained by surveys of large samples of young stars. These surveys probe the inner disk regions using excess emission above the stellar photosphere at infrared wavelengths and find that the lifetime is several megayears (Strom et al. 1989b; Haisch et al. 2001; Hillenbrand 2002; Hernández et al. 2007b; Sicilia-Aguilar et al. 2006a; Fang et al. 2013). The large variety of disk morphologies observed at a given age suggests that the disk evolution is controlled by several parameters: stellar and disk mass, multiplicity, local environment, etc. (Hartmann et al. 2006; Bouwman et al. 2006; Guarcello et al. 2007; Fang et al. 2009, 2012).

Observations at infrared wavelengths suggest that there are two types of evolved disks: radially depleted disks and globally depleted disks. The radially depleted disks show very weak or no infrared excess at near-infrared wavelengths but strong excess emission at mid-infrared and longer wavelengths, suggesting that dust in these disks is cleared starting from the inside and moving outward (Hayashi et al. 1985; Osterloh \& Beckwith 1995; Meyer \& Beckwith 2000; Mamajek et al. 2004; Andrews $\&$ Williams 2005; Carpenter et al. 2005). The globally depleted disks exhibit an approximately uniformly reduced infrared excess compared with primordial disks over all wavelengths out to $24 \mu \mathrm{m}$ (Lada et al. 2006; Sicilia-Aguilar et al. 2008, 2009; Currie \& Kenyon 2009; Currie et al. 2009; Currie 2010), indicating that there is global dust depletion in these disks. An investigation of these two types of evolved disks is important in order to understand disk dissipation processes.

Radially depleted disks, usually called transition disks (TDs), are better studied in the literature than globally depleted disks. In the following, we refer to only the radially depleted disks as TDs. Several processes have been proposed to produce TDs, including (1) giant planet formation (Rice et al. 2003; Quillen et al. 2004), (2) tidal truncation in close binaries, (3) photoevaporation (Johnstone et al. 1998; Störzer \& Hollenbach 1999; Richling \& Yorke 2000; Hollenbach et al. 2000; Clarke et al. 2001; Armitage et al. 2003; Alexander et al. 2006), (4) magnetorotational instability (MRI; Chiang \& Murray-Clay 2007), and (5) dust grain growth (Sicilia-Aguilar et al. 2011). To distinguish among these mechanisms, an important diagnostic is the comparison of the accretion rate of matter onto the central star between TD objects and less evolved classical T Tauri stars (CTTSs; Najita et al. 2007; Sicilia-Aguilar et al. 2010; Cieza et al. 2010).

For normal CTTSs, various studies have suggested that there is an empirical correlation between the average accretion rate and the mass of the central star of $\dot{M}_{\text {acc }} \propto M_{*}^{\alpha}$, with $\alpha \approx 1-3$ (White \& Basri 2003; Muzerolle et al. 2003, 2005; Calvet et al. 2004; Mohanty et al. 2005; Natta et al. 2006; Garcia Lopez et al. 2006; Herczeg \& Hillenbrand 2008; Gatti et al. 2008; Fang et al. 2009). Although the correlation between accretion rate and stellar mass is obvious for a large sample 
of young stars, individual objects with similar ages and masses commonly scatter around the average relation by up to two orders of magnitude. Multiple epoch observations of the same stars can show large variations in the $\mathrm{H} \alpha$ emission strength and line profile, pointing to substantial temporal variations in the accretion (Johns \& Basri 1995; Gullbring et al. 1996; Alencar et al. 2001; Alencar \& Batalha 2002; Sicilia-Aguilar et al. 2010), which provides one plausible explanation for the large scatter in the $\dot{M}_{\text {acc }}$ versus $M_{*}$ relation. Other explanations for such a large scatter relate to initial conditions, angular momentum, disk mass, and substantial time evolution (Hartmann et al. 1998; Dullemond et al. 2006).

To address all of these issues, we need to study a large sample of well-characterized young stars. Lynds 1641 (L1641) is one of the best sites for such an investigation. L1641 is located in the Orion molecular cloud complex at a distance of 400-500 pc (e.g., Anthony-Twarog 1982; Hirota et al. 2007), and the region probably has a "depth" of at least several tens of parsecs. In this work, we will assume a distance of $450 \mathrm{pc}$. In L1641, a large population of young stars is formed in relative isolation, in addition to a population of young stars in many clusters or aggregates (Strom et al. 1993; Allen 1995; Fang et al. 2009). Spitzer observations of this region have yielded excellent samples of thousands of young stars down to very low mass including substantial numbers of TDs as well as normal T Tauri stars (Fang et al. 2009; Megeath et al. 2012). In Fang et al. (2009, hereafter Paper I), we performed a large spectroscopic survey of young stellar objects (YSOs) in L1641 with the Very Large Telescope (VLT)/VIMOS. Using these data in combination with the optical and near-infrared photometry, we derived the masses, ages, and accretion properties of a subsample of YSOs in L1641, and related these properties to their disk properties traced by the Spitzer observations. Following the work in Paper I, we perform a new optical spectroscopic survey of young stars in the L1641 cloud with the MMT/Hectospec and the MMT/Hectochelle. In this work, we report on the results from these observations. The data from Hectospec can cover a wavelength range from $3700 \AA$ to $9000 \AA$, and are used to confirm the youth and determine the spectral types of YSO candidates in L1641, especially for the TD candidates proposed in Paper I. Furthermore, the spectra from Hectochelle can cover the $\mathrm{H} \alpha$ line with high spectral resolution. In this work, a group of YSOs in L1641 have been observed at multiple epochs with Hectochelle, aimed at monitoring the accretion variations of YSOs in L1641.

This paper is organized as follows: in Section 2 we describe our observations and data reduction, in Section 3 we delineate our data analysis, we present our results in Section 4 followed by a discussion in Section 5, and we summarize our efforts in Section 6.

\section{OBSERVATIONS AND DATA REDUCTION}

We combine newly obtained spectroscopic data from Hectospec (Fabricant et al. 2005) and Hectochelle (Szentgyorgyi et al. 2011) with photometric data from Paper I. We use the spectroscopic data to determine the spectral classification and characterize the accretion properties of YSOs in L1641.

\subsection{Photometry}

Our photometric data used in this work are mainly extracted from Paper I (see the description in Paper I). We complement them with data from the UKIRT Infrared Deep Sky Survey
(UKIDSS; Lawrence et al. 2007) and the Wide-field Infrared Survey Explorer (WISE; Wright et al. 2010). We briefly describe each data set below.

Our optical photometry is partially taken from the Sloan Digital Sky Survey (SDSS; York et al. 2000) in the $u^{\prime} g^{\prime} r^{\prime} i^{\prime} z^{\prime}$ bands centered on $0.35,0.48,0.62,0.76$, and $0.91 \mu \mathrm{m}$, respectively. The $10 \sigma$ limiting magnitudes for the SDSS survey of the L1641 cloud are $\sim 20.5,21.7,21.4,21.1$, and 19.7 in the $u^{\prime} g^{\prime} r^{\prime} i^{\prime} z^{\prime}$ bands, respectively. The SDSS survey has not covered the southeast half of the L1641 cloud toward which we complemented the SDSS photometry of L1641 with CCD imaging in the SDSS $g^{\prime} r^{\prime} i^{\prime} z^{\prime}$ bands performed at the Calar Alto $3.5 \mathrm{~m}$ telescope, using the Large Area Imager for Calar Alto (LAICA). Conditions were photometric, but the seeing was poor $\left(2^{\prime \prime}-3^{\prime \prime}\right)$, somewhat limiting the sensitivity for faint point sources $(\sim 19.6,19.6,19.9$, and 18.9 mag at $g^{\prime} r^{\prime} i^{\prime} z^{\prime}$ bands, respectively).

The near-infrared photometry in the $J H K_{\mathrm{S}}$ bands was taken from the Two Micron All Sky Survey (2MASS; Skrutskie et al. 2006), with $10 \sigma$ limiting magnitudes of $\sim 16.2,15.3$, and $14.6 \mathrm{mag}$, respectively. The northwest part of the L1641 cloud (decl. (J2000)> -6.44) has been observed by UKIDSS (Lawrence et al. 2007), in the $J H K$ bands, with $10 \sigma$ limiting magnitudes of $\sim 19.3,18.2$, and $17.6 \mathrm{mag}$, respectively. For a few objects which are too faint to be detected in the 2MASS survey, we extract their photometry in the UKIDSS catalog.

The mid-infrared photometry for the sources in the L1641 cloud is extracted from the Spitzer IRAC and MIPS imaging data (see the detailed description for the data reduction in Paper I). The $10 \sigma$ limiting magnitudes for the IRAC and MIPS imaging survey of the L1641 cloud are $\sim 17.5,17.2,14.4,12.2$, and $9.0 \mathrm{mag}$ in the IRAC [3.6], [4.5], [5.8], [8.0], and [24] bands, respectively. The L1641 cloud has been covered by the WISE survey at wavelengths of $3.4,4.6,12$, and $22 \mu \mathrm{m}$ with spatial resolutions of 6". 1, 6.'4, 6.'5, and 12'. 0 . We also extracted the midinfrared photometry from the WISE survey. The $10 \sigma$ limiting magnitudes are estimated to be $\sim 16.4,15.1,10.7$, and 7.1 mag in the WISE [3.4], [4.6], [12], and [22] bands, respectively.

\subsection{X-Ray Emission}

Young stars can be identified by their X-ray emission, which is two to three orders of magnitude brighter than seen in the field population (Feigelson \& Montmerle 1999). Thus, complementary X-ray data can be extremely useful to distinguish young stars from field stars in star-forming regions. The L1641 cloud has been almost fully covered by the $X M M$ survey (Wolk 2009). The sky coverage of the $X M M$ survey is shown in Figure 2. All the X-ray emission sources detected in the survey have been published in the XMM-Newton Serendipitous Source Catalogue (Watson et al. 2009). We have extracted $~ 940$ X-ray sources from the XMM Serendipitous Source Catalogue in the field of L1641. We matched the X-ray sources to our targets using $2^{\prime \prime}$ tolerance, and found optical and/or infrared counterparts for $66 \%$ of the X-ray sources. We consider these X-ray emission sources to be likely YSO candidates. We must stress that a small fraction of "YSOs" in our catalog can be bright active galactic nuclei (AGNs) and nearby foreground stars. Without spectroscopic data, these sources are difficult to exclude.

\subsection{Optical Spectroscopy}

\subsubsection{Target Selection}

We selected targets for our spectroscopic observations if they obeyed any of the following criteria. 


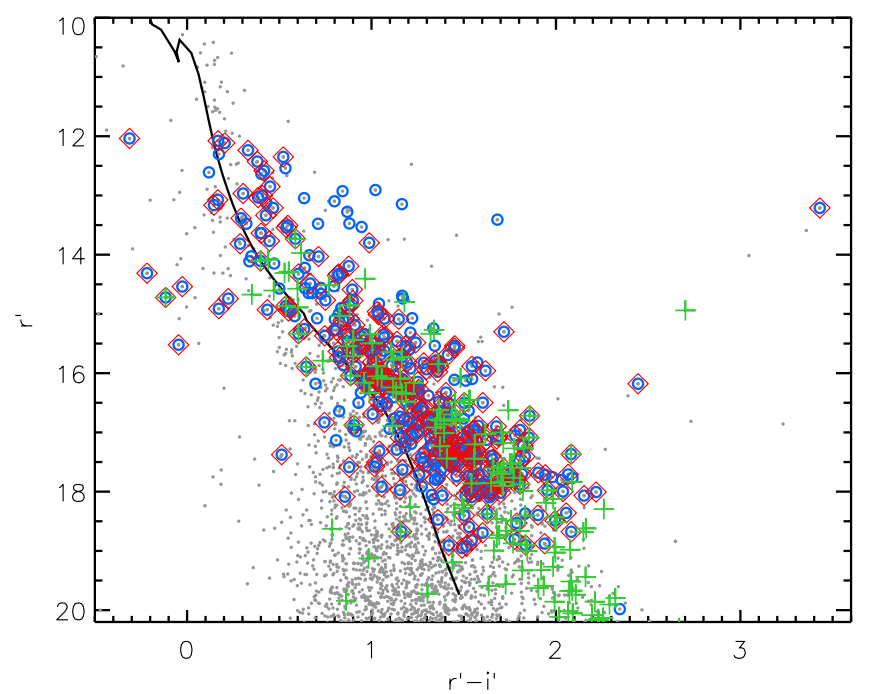

Figure 1. $r^{\prime}$ vs. $r^{\prime}-i^{\prime}$ color-magnitude diagram. The gray-filled circles show all the sources found in the L1641 field (Paper I), the open circles and diamonds are the targets for the spectroscopic surveys with Hectospec and Hectochelle, respectively. The pluses mark the YSOs in Paper I. The solid line shows the $10 \mathrm{Myr}$ pre-main-sequence isochrone from Dotter et al. (2008).

(A color version of this figure is available in the online journal.)

1. They have X-ray emission.

2. They have infrared excess.

3. They are above the 10 Myr PMS isochrone.

To reach a sufficient signal-to-noise ratio $(\mathrm{S} / \mathrm{N})$ for spectral classification, we selected the sources with SDSS $r^{\prime}$ magnitude brighter than $\sim 19$ mag. One additional target, fainter than 19 mag in the $r^{\prime}$ band but very bright in the $i^{\prime}$ band, is also included in our spectroscopic sample from Hectospec. For the spectroscopic survey with Hectochelle, we selected the YSOs with SDSS $r^{\prime} \sim 12-19$ mag. Figure 1 shows the $r^{\prime}$ versus $r^{\prime}-i^{\prime}$ color-magnitude diagram of our targets for both spectroscopic surveys.

\subsubsection{Spectroscopic Observations and Data Reduction}

The intermediate-resolution spectra of sources were taken with the Hectospec multi-object spectrograph which can take a maximum of 300 spectra simultaneously. We used the 270 groove $\mathrm{mm}^{-1}$ grating and obtained spectra in the range $3700-9000 \AA$ with a resolution of $\sim 5 \AA$. We have $\sim 450$ targets, which are distributed at three pointings (see Figure 2). The observational data were taken on the nights of 2011 January 23, 24 , and 25. Table 1 lists the observational logs.

The high-resolution $\mathrm{H} \alpha$ spectroscopy of sources was obtained with the Hectochelle multi-object spectrograph which can take a maximum of 240 spectra simultaneously with a spectral resolution of 34,000 . We used the OB 26 filter which simultaneously covers $\mathrm{H} \alpha$ emission line and the $\mathrm{Li}$ I $\lambda 6708$ absorption line. The observational data were taken on the night of 2010 February 5 with two pointings. On the nights of 2010 March 3, 2010 November 29, and 2011 October 19, a major fraction of our targets was observed again. The observational logs are listed in Table 1.

Toward each pointing observed with Hectochelle or Hectospec, we have taken at least one extra set of sky spectra

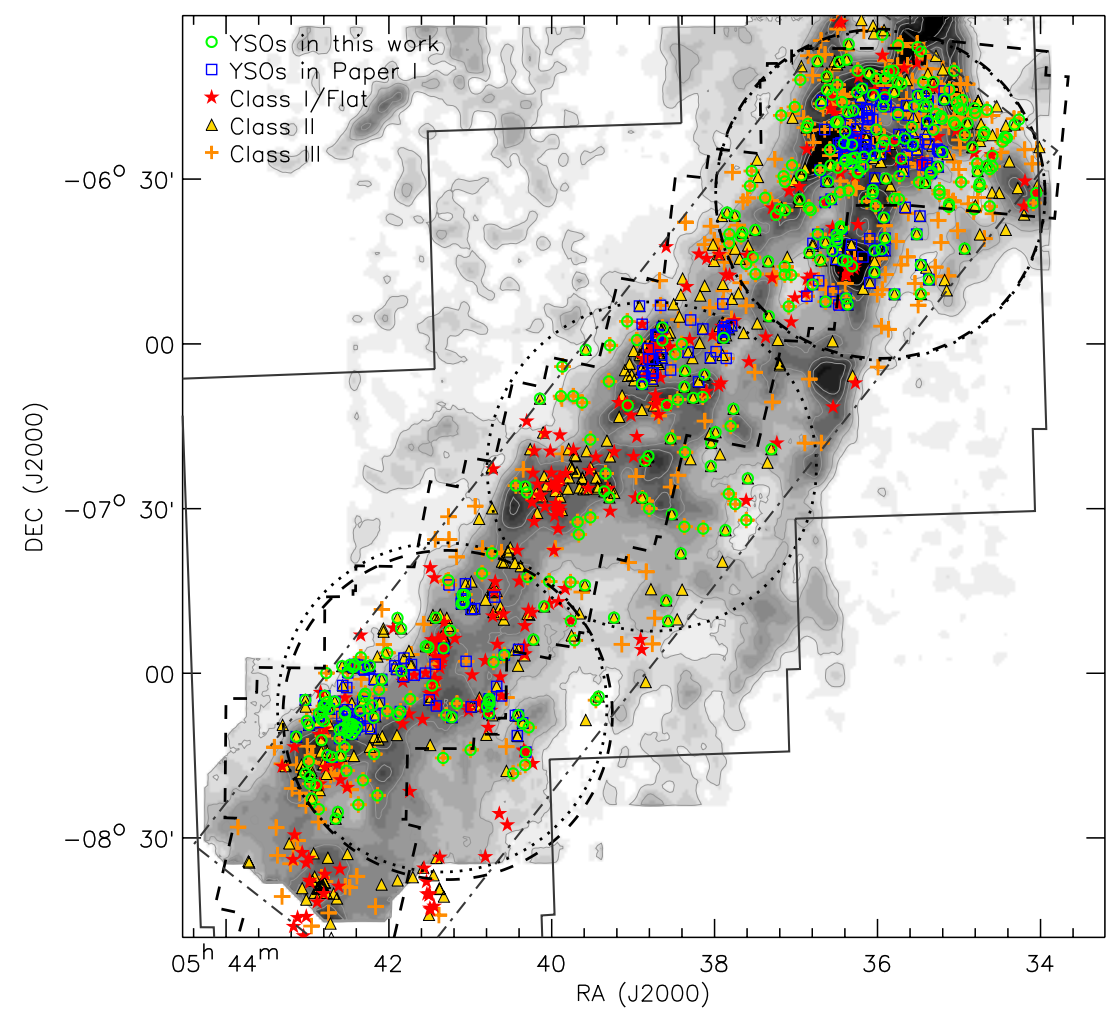

Figure 2. Distribution of YSOs in L1641 overplotted on a ${ }^{13} \mathrm{CO}$ integrated intensity map which is a combination of our observations with the Delingha $14 \mathrm{~m}$ telescope and data from Bally et al. (1987). The green open circles mark the YSOs observed with Hectospec. The blue open squares show the YSOs studied in Paper I. The red filled star and yellow filled triangles represent the Class I/flat-spectrum and Class II YSOs, respectively. The orange pluses show the Class III sources. The large dotted-line circles show the FOVs of our spectroscopic surveys with Hectospec, and the large dashed-line circles display the FOVs of our Hectochelle survey. The dashed lines enclose the fields observed with XMM. The dash-dotted lines encircle the regions observed with Spitzer IRAC, and the solid lines enclose the fields observed with Spitzer MIPS $24 \mu \mathrm{m}$.

(A color version of this figure is available in the online journal.) 
Table 1

Observation Logs

\begin{tabular}{|c|c|c|c|c|c|c|}
\hline \multirow[b]{2}{*}{ Config. } & \multirow[b]{2}{*}{ Obs-date } & \multirow{2}{*}{$\begin{array}{c}\text { R.A. } \\
\text { (J2000) } \\
\text { (h:m:s) }\end{array}$} & \multirow{2}{*}{$\begin{array}{c}\text { Decl. } \\
(\mathrm{J} 2000) \\
(\mathrm{d}: \mathrm{m}: \mathrm{s})\end{array}$} & \multicolumn{2}{|c|}{ Exposure } & \multirow[b]{2}{*}{$\begin{array}{l}\text { Offset }^{\mathrm{a}} \\
(\operatorname{arcsec})\end{array}$} \\
\hline & & & & $\begin{array}{c}\text { Object } \\
\text { (minutes) }\end{array}$ & $\begin{array}{c}\text { Sky } \\
\text { (minutes) }\end{array}$ & \\
\hline \multicolumn{7}{|c|}{ Hectochelle } \\
\hline \multirow[t]{3}{*}{1} & 2010 Feb 5 & 05:36:01 & $-06: 32: 24$ & $3 \times 30$ & $1 \times 30$ & 5 \\
\hline & 2010 Mar 3 & 05:36:01 & $-06: 32: 24$ & $3 \times 30$ & $1 \times 30$ & 5 \\
\hline & 2010 Nov 29 & $05: 36: 01$ & $-06: 34: 59$ & $4 \times 30$ & $1 \times 30$ & 7 \\
\hline \multirow[t]{2}{*}{2} & 2010 Feb 5 & $05: 41: 17$ & $-08: 07: 33$ & $3 \times 30$ & $1 \times 30$ & 5 \\
\hline & 2011 Oct 19 & $05: 41: 17$ & $-08: 07: 33$ & $8 \times 20$ & $1 \times 20$ & 5 \\
\hline \multicolumn{7}{|c|}{ Hectospec } \\
\hline 3 & 2011 Jan 23 & 05:36:01 & -063233 & $6 \times 20$ & $2 \times 20$ & 10 \\
\hline 4 & 2011 Jan 24 & $05: 41: 21$ & -080610 & $4 \times 10$ & $1 \times 10$ & 5 \\
\hline 5 & 2011 Jan 25 & $05: 39: 12$ & -072446 & $5 \times 10$ & $1 \times 10$ & 6 \\
\hline
\end{tabular}

Note.

a The offsets between the pointings of object exposures and sky exposures.

by offsetting the telescope by $\sim 5^{\prime \prime}-10^{\prime \prime}$ between the science exposures, which can provide us with a sky spectrum close to each object. Together with the rest of the sky fibers at each science exposure, this enables us to construct the appropriate nebular and sky spectrum for subtraction, taking into account the different transmission of every fiber and the variability of the nebular and sky emission throughout the field of view (FOV).

We used IRAF routines to reduce the Hectospec and Hectochelle data according to a standard procedure. We flattened and extracted the spectra using dome flats with the IRAF task dofibers under the package specred. The wavelength solution was achieved with HeNeAr and ThAr comparison spectra for Hectospec and Hectochelle, respectively, using the IRAF tasks identify and reidentify under the package specred. We calibrated the spectra with a wavelength solution constructed using the IRAF task dispcor under the package specred. For each pointing, we performed observations at several exposures. We then extracted the spectra for each exposure and finally, obtained the spectra for each target and the corresponding sky spectra close to the target. We subtracted the sky from the spectra of each target, and combined the sky-subtracted spectra into one final spectrum.

\subsubsection{Complementary Literature Spectroscopy}

An additional sample of 12 stars with spectral types, 10 of which are from Allen (1995) and 2 of which are from our VIMOS spectroscopic survey, is also included in this work. These stars show X-ray emission, but are not in Paper I. Thus, we include them in this work.

\section{ANALYSIS}

\subsection{YSO Selection Criteria}

A star in our sample is classified as a young star if it obeys any of the following: (1) X-ray emission, (2) infrared excess, or (3) $\mathrm{H} \alpha$ emission.

When good $\mathrm{S} / \mathrm{N}$ Hectochelle spectra are available, we also use the $\mathrm{Li}$ I $\lambda 6708$ absorption line as an indicator of youth. We find that sources with strong excess infrared emission always show $\mathrm{H} \alpha$ emission, but do not always show X-ray emission. We also find that some stars with X-ray emission show $\mathrm{H} \alpha$ absorption. These stars typically have spectral types from late-A to late-G. We note that there may be a small contamination of our sample from dMe stars, which are old, M-type stars that show $\mathrm{H} \alpha$ emission due to chromospheric activity, and from extragalactic sources which show infrared excess and/or X-ray emission.

\subsection{Spectral Classification}

We classified the Hectospec spectra using the scheme developed by Hernández et al. (2004), which employs the empirical relation between the equivalent widths (EWs) of selected atomic and molecular absorption lines and the effective temperature. The classification scheme consists of three sub-regions; each region combines a number of absorption features and spans a range of spectral types (see the detailed description of the method in Hernández et al. 2004).

In Figure 3, we show sample Hectospec spectra of stars with spectral types from late $\mathrm{G}$ to late $\mathrm{M}$ from our survey. There is a clear change in the spectral shape with spectral type, especially the $\mathrm{TiO}$ strength, which is a prominent diagnostic for late $\mathrm{K}$ - to M-type stars.

Of the $\sim 430$ targets with spectral-type estimates in our spectroscopic sample, $\sim 290$ sources have been classified in the literature (Paper I; Allen 1995; Gâlfalk \& Olofsson 2008; Hsu et al. 2012). Figure 4 shows the comparison of the spectral types in this work and those quoted in the literature. About $90 \%$ of this sample agrees within one subclass and $95 \%$ within two subclasses.

\subsection{Determining the Stellar Properties}

We converted the spectral types to effective temperatures using the relation from Kenyon \& Hartmann (1995) for stars earlier than M0, and the one from Luhman et al. (2003) for stars later than M0. We determined the extinction and bolometric luminosity of the central stars in the YSOs by fitting their optical and near-infrared photometry with a (reddened) model atmosphere with the effective temperature derived from spectral classification. We adopted models by Kurucz $(1979,1994)$ at temperatures above $4500 \mathrm{~K}$, and the MARCS models at lower temperatures (Gustafsson et al. 2008). The fitting employs two free parameters: the angular diameter $(\theta)$ and the extinction $\left(A_{\mathrm{V}}\right)$ in the $V$ band. In general, we use the photometry in $g^{\prime}$, $r^{\prime}, i^{\prime}, z^{\prime}$ from SDSS, and in $J$ bands for our spectral energy 


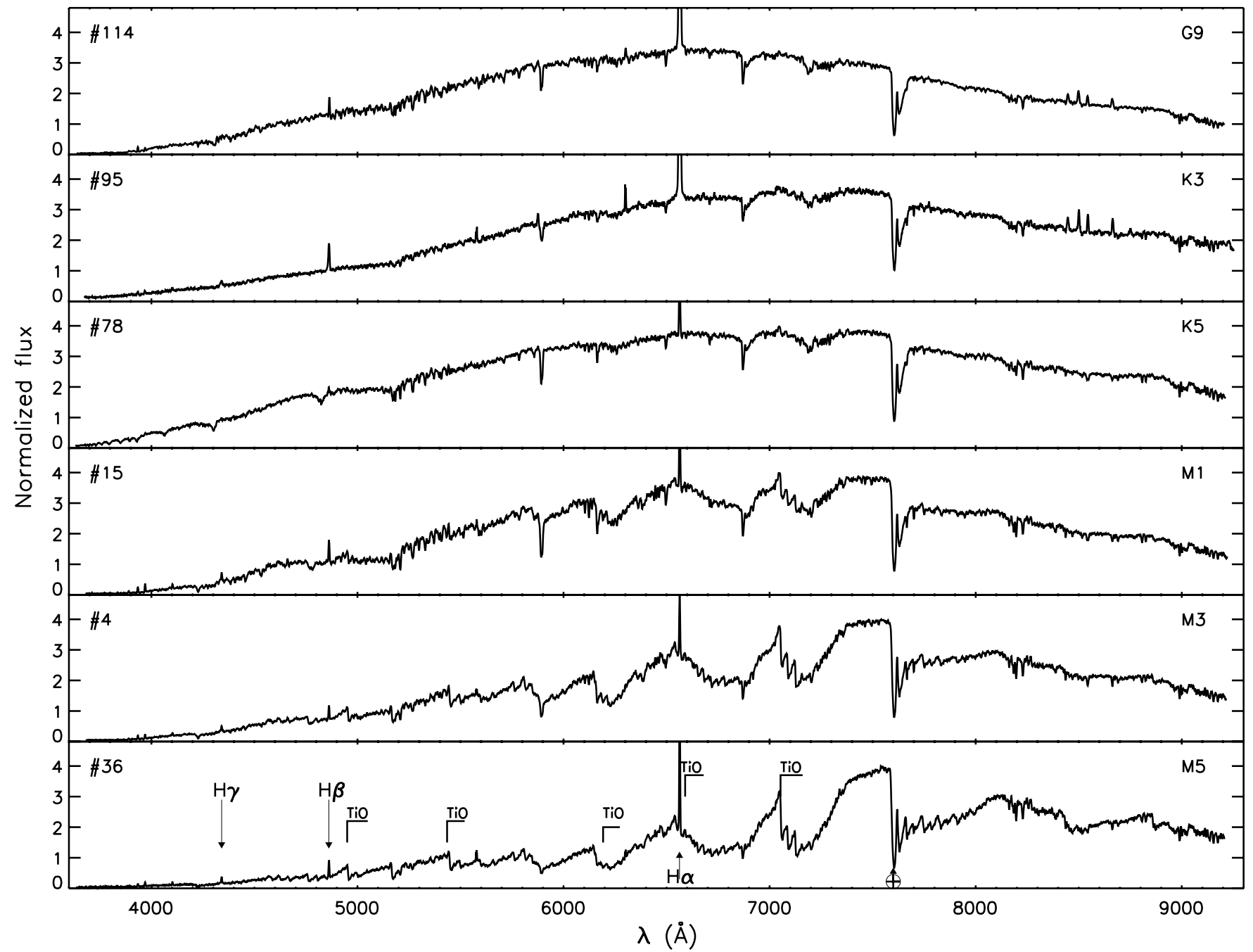

Figure 3. Example spectra from our Hectospec observations covering a range of spectral types. The prominent Balmer lines $(\mathrm{H} \alpha, \mathrm{H} \beta, \mathrm{H} \gamma)$ and TiO absorption features are indicated.
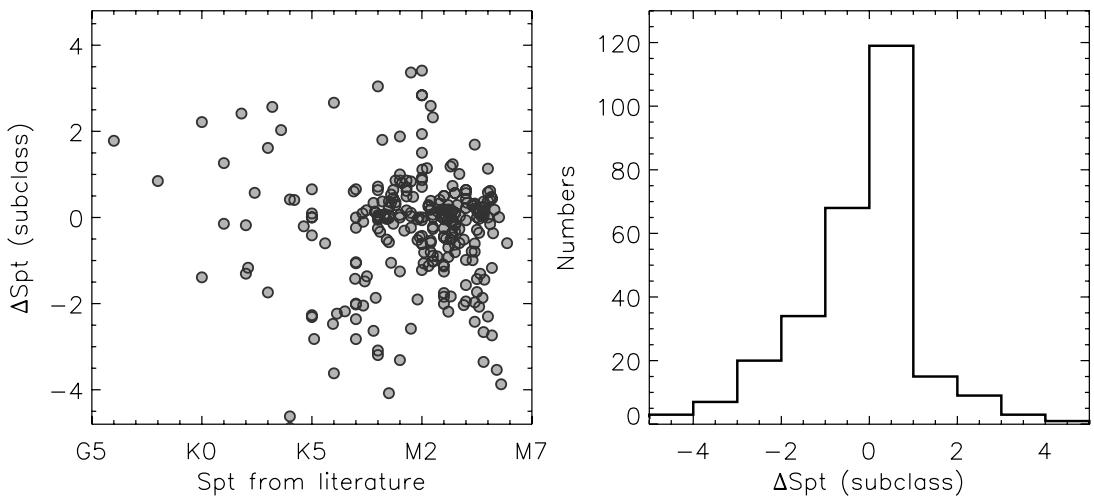

Figure 4. Left panel: the differences between the spectral types in this work and in the literature vs. their spectral types in the literature. Right panel: the distribution of spectral-type differences in the left panel.

distribution (SED) fit, but for stars without excess near-infrared emission we also included the $H$ and $K_{\mathrm{s}}$ bands. We calculated the synthetic photometry by integrating the intensity of the (reddened) model atmospheres over the spectral response curve of the system for each filter and compared them with the observations. By minimizing the $\chi^{2}$ in an automated iterative procedure, we obtained the optimum values for $\theta$ and $A_{\mathrm{V}}$. We used the extinction law of Cardelli et al. (1989) to redden model atmospheres, and adopted a total to selective extinction value typical of interstellar medium dust $\left(R_{\mathrm{V}}=3.1\right)$.

We calculated the bolometric luminosity of stars from the effective temperatures and angular diameters assuming a distance of 450 pc (Genzel et al. 1981; Anthony-Twarog 1982; Maddalena et al. 1986; Wilson et al. 2005), using the following formula:

$$
L_{\mathrm{bol}}=\pi \theta^{2} d^{2} \sigma T_{\mathrm{eff}}^{4},
$$


where $\theta$ is the angular diameter, $d$ is the distance, $\sigma$ is the Stefan-Boltzmann constant, and $T_{\text {eff }}$ is the effective temperature.

Using effective temperatures and bolometric luminosities, we can place the YSOs in the H-R diagram from which stellar masses and ages can be estimated by comparison to theoretical pre-main-sequence (PMS) evolutionary tracks. However, several sets of such tracks from various authors exist and these yield significantly different results for stellar ages (see, e.g., Hillenbrand et al. 2008, for a discussion). We estimated masses and ages using five different sets of publicly available PMS evolutionary tracks by D'Antona \& Mazzitelli (1997, DM97), Baraffe et al. (1998, B98), Siess et al. (2000, S00), Dotter et al. (2008, D08), and Tognelli et al. (2011, Pisa11). In the remainder of the discussion, unless otherwise noted, we adopt the values obtained from the tracks of Dotter et al. (2008), as these have the best resolution in both mass and age. We stress, however, that there are substantial systematic differences between the different sets of tracks (see Hillenbrand \& White 2004; Hillenbrand et al. 2008, for a detailed discussion of the various sets of PMS evolutionary tracks available in the literature), and our motives for choosing those by Dotter et al. (2008) are pragmatic.

We employ the same method as in Paper I to estimate the uncertainties in mass and age of the individual stars. We use a simple Monte Carlo method to create a large number of synthetic $\left[T_{\mathrm{eff}}, L_{*}\right]$ points for each star, assuming the errors in both quantities to be normally distributed. For each point in the H-R diagram, we derived the mass and age, and use the standard deviations in the resulting mass and age distribution as their uncertainties. This procedure can account for the observational errors, but the systematic uncertainties from different PMS evolution tracks remain. In addition, for highly extincted sources, the shape of the adopted extinction law can affect the resulting mass and age by affecting the stellar luminosity estimate. Higher values for the total to selective extinction $R_{\mathrm{V}}$ can lead to higher stellar luminosities, which yield younger ages for YSOs, and higher masses for YSOs with earlier spectral types.

\subsection{Determining the Disk Properties}

\subsubsection{Infrared Spectral Slopes and Classifications}

We calculate the infrared spectral slope for each YSO, defined as $\alpha=d \log \left(\lambda F_{\lambda}\right) / d \log (\lambda)$, with the dereddened photometry in the infrared broad bands. We calculate four sets of infrared slopes, $\alpha_{2-8}, \alpha_{3.6-8}, \alpha_{2-24}$, and $\alpha_{3.6-24}$, corresponding to the spectral ranges of $K_{\mathrm{s}}$ to [8.0], [3.6] to [8.0], $K_{\mathrm{s}}$ to [24], and [3.6] to [24], respectively. The extinction of each YSO is estimated individually. For the YSOs with spectral-type measurements, we can accurately estimate their extinction by fitting their optical and near-infrared photometry as described in Section 3.3. However, for the YSOs without spectral types, we can only roughly determine their extinction with a method similar to that used by Gutermuth et al. (2008), in which the extinction is obtained by employing the $H-K_{\text {s }}$ versus $J-H$ color-color diagram. We describe the method below.

The location of each YSO in the $H-K_{\mathrm{s}}$ versus $J-H$ color-color diagram depends on both its intrinsic colors and its extinction. To estimate the extinction of individual YSOs, we need to have knowledge of their intrinsic colors. For the diskless YSOs, their intrinsic colors are mainly determined by their spectral types. For YSOs with disks, the excess emissions from hot inner regions of disks can also contribute to their

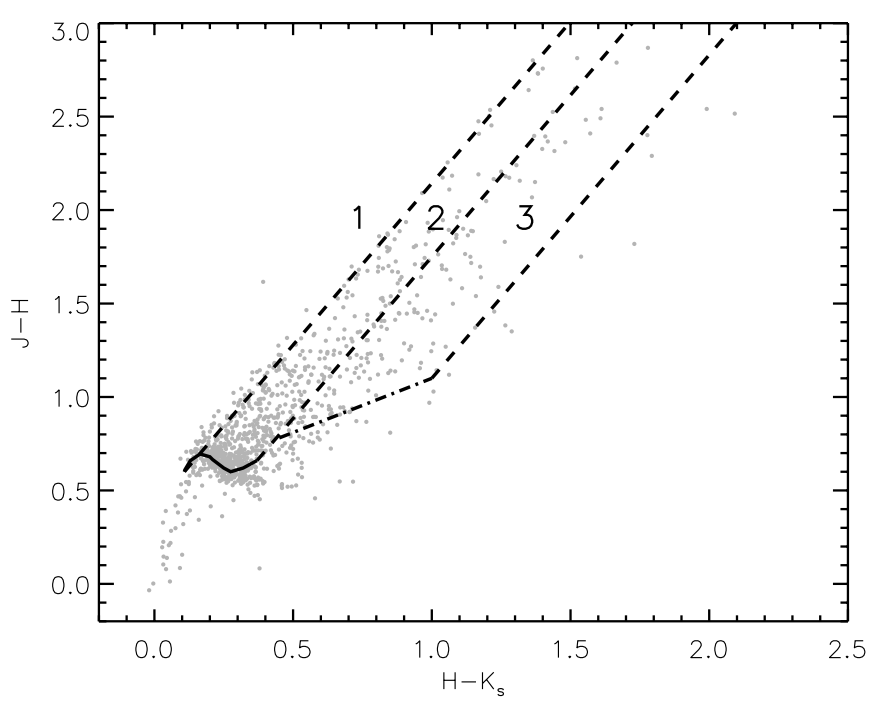

Figure 5. $H-K_{\mathrm{s}}$ vs. $J-H$ color-color diagram for the young stars in L1641. The solid line shows the intrinsic colors for the main-sequence stars of $\sim$ K5-M6 type (Bessell \& Brett 1988), and the dash-dotted lines are the locus of T Tauri stars from Meyer et al. (1997). The dashed lines show the reddening vectors from Flaherty et al. (2007). The dashed lines separate the diagram into three regions marked as 1, 2, and 3 in the figure. In each region, we use different ways to estimate the extinction of the sources without spectral types.

intrinsic colors and make them redder than diskless stars. The CTTS locus in the $H-K_{\mathrm{s}}$ versus $J-H$ color-color diagram is observationally defined as the colors of dereddened CTTSs. In consideration of these different origins of intrinsic colors of YSOs, we divide the diagram into three sub-regions (see Figure 5). For each region, we use a different method to obtain the intrinsic color $[J-H]_{0}$ of the sources. Extinction values of individual YSOs are estimated from their observed $[J-H]\left([J-H]_{\text {obs }}\right)$ and $[J-H]_{0}$ using the equation $A_{\mathrm{K}}=\left([J-H]_{\mathrm{obs}}-[J-H]_{0}\right) / 0.95$. In Region 1 , the sources show slightly bluer $[J-H]$ colors than expected from normal reddening of diskless YSOs, probably due to the photometric uncertainties. For such YSOs, their $[J-H]_{0}$ values are simply assumed to be 0.6 (the typical value for a K5-type dwarf star). In Region 2, the color excess of each source is mainly due to reddening. Its intrinsic $[J-H]$ color is obtained from the intersection between the reddening vector and the locus of mainsequence stars. ${ }^{6}$ In Region 3, the sources are supposed to be the reddened CTTSs. The intrinsic $[J-H]$ color is derived from where the reddening vector and the CTTS locus intersects. For the stars outside of these three regions or without detection in all three 2MASS bands, their extinction is assumed to be zero. The extinction values derived from the $H-K_{\mathrm{s}}$ versus $J-H$ color-color diagram are less accurate than those of YSOs with spectral types. For a sample of spectroscopic YSOs with substantial extinction $\left(A_{\mathrm{V}}>1\right)$, we compare the extinctions estimated from the above rough method with the more accurate ones described in Section 3.3. We find that the extinctions from the rough method are statistically slightly lower than the relatively accurate ones. We estimate how the extinction affects the spectral slopes. For $A_{\mathrm{K}}=1$, the dereddened $\alpha_{2-8}, \alpha_{3.6-8}$, $\alpha_{2-24}$, and $\alpha_{3.6-24}$ are $0.36,0.14,0.15$, and 0.04 less than the observed spectral slopes, respectively. Thus, given the typical extinction for our YSOs in L1641, extinction only slightly influences our estimates of the spectral slopes of the YSOs, except for a few extremely embedded YSOs.

\footnotetext{
6 For simplicity, we force $[J-H]_{0} \geqslant 0.6$ as in Gutermuth et al. (2008).
} 
We classify the YSOs into different classes according to the two spectral slopes, i.e., $\alpha_{3.6-8}$ and $\alpha_{3.6-24}$. For the sources with MIPS $24 \mu \mathrm{m}$ detection, we classify the sources as diskless stars if $\alpha_{3.6-24} \leqslant-2.2$, Class II sources if $-2.2<\alpha_{3.6-24}<-0.3$, flat-spectrum when $-0.3 \leqslant \alpha_{3.6-24} \leqslant+0.3$, and Class I sources if $\alpha_{3.6-24}>+0.3$ (Luhman et al. 2008). Some YSOs have not been detected at $24 \mu \mathrm{m}$. For these YSOs, we use the dereddened infrared slope $\left(\alpha_{3.6-8}\right)$ to classify the young stars as Class III sources if $\alpha_{3.6-8} \leqslant-2.56$, Class II sources if $-2.56<\alpha_{3.6-8}<$ -0.3 , flat-spectrum sources when $-0.3 \leqslant \alpha_{3.6-8.0} \leqslant+0.3$, and Class I sources if $\alpha_{3.6-8.0}>+0.3$. For 857 YSOs with reliable estimates of spectral types, their classes will be revised by comparing their reddened model photospheric fluxes with their observed fluxes at infrared wavelengths.

\subsubsection{Accretion Rates}

Gas from the disk accretes onto the star along the magnetic field lines and hits the stellar surface at approximately the freefall velocity, causing a strong accretion shock and associated hot spots on the stellar surface. Various emission lines, such as the hydrogen Balmer series, He I $5876 \AA$, Br $\gamma$, etc., are formed in the infalling magnetospheric flow (Hartmann et al. 1994; Gullbring et al. 1998; Muzerolle et al. 2001; Lima et al. 2010; Kurosawa et al. 2011). Optical/ultraviolet excess continuum emission is produced in the accretion shocks. All these emission lines or excess emission can be used to estimate the accretion rates with the help of models or the empirical relation between line luminosity and accretion luminosity (Paper I; Hartmann et al. 1994; Hartigan et al. 1995; Gullbring et al. 1998; Muzerolle et al. 2001; Calvet et al. 2004; Natta et al. 2004; Herczeg \& Hillenbrand 2008).

We use two methods to estimate the accretion rates: (1) the empirical relation between accretion luminosity and $\mathrm{H} \alpha$ or $\mathrm{H} \beta$ line luminosity from Paper I and (2) the empirical relation between the full width of $\mathrm{H} \alpha$ at $10 \%\left(\mathrm{FW}_{\mathrm{H} \alpha, 10 \%}\right)$ of the peak intensity and accretion rates from Natta et al. (2004).

In the former method, we estimate the accretion rates from the observed $\mathrm{H} \alpha$ and $\mathrm{H} \beta$ line luminosities. The $\mathrm{H} \alpha$ and $\mathrm{H} \beta$ line luminosities are calculated by integrating over the line profile, adopting the best-fit model atmosphere spectrum (see Section 3.3) as the continuum level. The line luminosities are converted to the accretion luminosity via the empirical relation given in Paper I:

$\log \left(L_{\mathrm{acc}} / L_{\odot}\right)=(2.27 \pm 0.23)+(1.25 \pm 0.07) \times \log \left(L_{\mathrm{H} \alpha} / L_{\odot}\right)$,

$\log \left(L_{\mathrm{acc}} / L_{\odot}\right)=(3.01 \pm 0.19)+(1.28 \pm 0.05) \times \log \left(L_{\mathrm{H} \beta} / L_{\odot}\right)$.

The inferred accretion luminosities are then converted into mass accretion rates using the following relation:

$$
\dot{M}_{\mathrm{acc}}=\frac{L_{\mathrm{acc}} R_{\star}}{G M_{\star}\left(1-\frac{R_{\star}}{R_{\mathrm{in}}}\right)},
$$

where $R_{\text {in }}$ denotes the truncation radius of the disk, which is taken to be $5 R_{\star}$ (Gullbring et al. 1998). $G$ is the gravitational constant, $M_{\star}$ is the stellar mass as estimated from the location of each star in the H-R diagram, and $R_{\star}$ is the stellar radius derived using the fitting procedure described in Section 3.3.

For the YSOs with high-resolution $\mathrm{H} \alpha$ spectroscopy, we distinguish accretors from non-accretors based on the criterion $\left(\mathrm{FW}_{\mathrm{H} \alpha, 10 \%}>250 \mathrm{~km} \mathrm{~s}^{-1}\right)$ described in Appendix A, and estimate the accretion rates with the empirical relation between accretion rates and $\mathrm{FW}_{\mathrm{H} \alpha, 10 \%}$, using the formula given by Natta et al. (2004):

$$
\log \dot{M}_{\mathrm{acc}}=-12.89( \pm 0.3)+9.7( \pm 0.7) \times 10^{-3} \mathrm{FW}_{\mathrm{H} \alpha, 10 \%} .
$$

A comparison of accretion rates derived from the above two methods is discussed in Appendix B.

\section{RESULTS}

\subsection{YSOs in L1641}

In this section, we will perform a census of YSOs in L1641 based on the data collected from several projects. Then, we will calculate the infrared spectral slopes of these YSOs, and classify them into different evolutionary stages.

\subsubsection{A Census of YSOs}

As one of the best studied star-forming regions in the literature, L1641 has been observed by various projects, including infrared imaging surveys (Strom et al. 1993; Megeath et al. 2012), spectroscopic surveys (Paper I; Allen 1995), and an X-ray emission survey (Wolk 2009; Watson et al. 2009, see FOVs of each survey in Figure 2). However, until now, no census of YSOs in this region has been available in the literature. In this work, we provide an inventory of YSO candidates according to the following selection criteria: (1) confirmed with spectroscopy in Paper I and this work, (2) showing excess infrared emission, or (3) showing X-ray emission. A source is considered to be a YSO if it obeys one of the above criteria. Based on this, we have identified 1247 YSOs.

Recently, Hsu et al. (2012) presented a catalog of $\sim 860$ young stars in L1641 confirmed with optical spectroscopy, among which $\sim 720$ stars fall within the region that we study in this paper. We compare our YSO sample with that of Hsu et al. (2012), and find $\sim 580$ sources common to both catalogs. About 140 YSOs in the catalog of Hsu et al. (2012) are absent in our census. Among those, $\sim 120$ sources are diskless stars and $\sim 20$ sources are YSOs with excess infrared emission. We did not identify these diskless YSOs because they do not show X-ray emission and have not been observed with spectroscopy prior to the survey of Hsu et al. (2012). The 20 disked YSOs are located close to the edge of the coverage of the Spitzer survey, and lack photometry in several IRAC bands in our previous photometric catalog. Here, we include all these additional YSOs in our YSO catalog; Tables 2 and 3 list the YSO criteria met by these sources as well as the optical and infrared photometry.

In our YSO catalog, there are 1273 sources with photometry in all four IRAC bands. Figure 6(a) shows their [3.6] - [4.5] versus [5.8]-[8.0] color-color diagram. The sources in the color-color diagram fall into two populations. A major fraction of stars are located toward the top right of the origin due to excess infrared emission from hot dust in the inner disk, while other sources reside in a cluster near the origin, consistent with photospheric infrared colors. In Figure 6(a), there are five sources (1074, $1218,1220,1233$, and 1244) showing IRAC colors distinct from the others. Those sources will be discussed in Section 4.5.1.

Figure 6(b) shows the $K_{\mathrm{s}}-[5.8]$ versus [8.0] - [24] color-color diagram. Due to the lower sensitivity of MIPS, only a few diskless stars are detected at $24 \mu \mathrm{m}$, and appear around the origin in Figure 6(b). A major fraction of YSOs detected at $24 \mu \mathrm{m}$ are harboring optically thick inner disks and are located 
Table 2

The Optical and Near-infrared Photometric Magnitudes of the YSOs in L1641

\begin{tabular}{|c|c|c|c|c|c|c|c|c|c|c|c|c|c|c|c|c|}
\hline ID & $\begin{array}{l}\text { R.A. } \\
\text { (J2000) }\end{array}$ & $\begin{array}{c}\text { Decl. } \\
\text { (J2000) }\end{array}$ & $\begin{array}{c}u^{\prime} \\
(\mathrm{mag})\end{array}$ & $\begin{array}{c}g^{\prime} \\
(\mathrm{mag})\end{array}$ & $\begin{array}{c}r^{\prime} \\
(\mathrm{mag})\end{array}$ & $\begin{array}{c}i^{\prime} \\
(\mathrm{mag})\end{array}$ & $\begin{array}{c}z^{\prime} \\
(\mathrm{mag})\end{array}$ & INS1 & $\begin{array}{c}J \\
(\mathrm{mag})\end{array}$ & $\begin{array}{c}H \\
(\mathrm{mag})\end{array}$ & $\begin{array}{c}K_{S} \\
(\mathrm{mag})\end{array}$ & INS2 & $\begin{array}{c}A_{\mathrm{V}} \\
(\mathrm{mag})\end{array}$ & $\mathrm{Sp}$ & IRE & X-Ray \\
\hline 1 & 053605.00 & -064243.8 & $20.65 \pm 0.08$ & $18.54 \pm 0.02$ & $17.09 \pm 0.02$ & $15.25 \pm 0.01$ & $14.06 \pm 0.01$ & SDSS & $12.31 \pm 0.02$ & $11.65 \pm 0.03$ & $11.30 \pm 0.02$ & 2MASS & 0.0 & $\mathrm{Y}$ & $\mathrm{Y}$ & $\mathrm{N}$ \\
\hline 2 & 053510.69 & -063415.5 & $22.47 \pm 0.35$ & $19.08 \pm 0.02$ & $17.66 \pm 0.02$ & $16.03 \pm 0.01$ & $15.20 \pm 0.03$ & SDSS & $13.76 \pm 0.02$ & $12.97 \pm 0.04$ & $12.38 \pm 0.02$ & 2MASS & 0.0 & $\mathrm{Y}$ & $\mathrm{Y}$ & $\mathrm{Y}$ \\
\hline 3 & 053452.29 & -063508.5 & $21.16 \pm 0.11$ & $18.79 \pm 0.02$ & $17.40 \pm 0.03$ & $15.76 \pm 0.02$ & $14.87 \pm 0.04$ & SDSS & $13.28 \pm 0.04$ & $12.74 \pm 0.05$ & $12.43 \pm 0.03$ & 2MASS & 0.0 & $\mathrm{Y}$ & $\mathrm{N}$ & $\mathrm{Y}$ \\
\hline 4 & 053550.56 & -063317.3 & $19.83 \pm 0.05$ & $17.39 \pm 0.01$ & $16.01 \pm 0.01$ & $14.80 \pm 0.01$ & $14.07 \pm 0.01$ & SDSS & $12.61 \pm 0.02$ & $11.96 \pm 0.03$ & $11.73 \pm 0.02$ & 2MASS & 0.0 & $\mathrm{Y}$ & $\mathrm{N}$ & $\mathrm{Y}$ \\
\hline 5 & 053510.75 & -063444.5 & $12.78 \pm 0.01$ & $14.89 \pm 0.01$ & $14.78 \pm 0.03$ & $14.88 \pm 0.02$ & $12.48 \pm 0.03$ & SDSS & $10.41 \pm 0.02$ & $10.19 \pm 0.03$ & $10.15 \pm 0.02$ & 2MASS & 0.3 & $\mathrm{Y}$ & $\mathrm{N}$ & $\mathrm{Y}$ \\
\hline
\end{tabular}

(This table is available in its entirety in a machine-readable form in the online journal. A portion is shown here for guidance regarding its form and content.) 
Table 3

The Infrared Photometric Magnitudes of the YSOs in L1641

\begin{tabular}{|c|c|c|c|c|c|c|c|c|c|c|c|c|c|c|c|c|}
\hline ID & $\begin{array}{l}\text { R.A. } \\
\text { (J2000) }\end{array}$ & $\begin{array}{l}\text { Decl. } \\
\text { (J2000) }\end{array}$ & $\begin{array}{c}W 1 \\
(\mathrm{mag})\end{array}$ & $\begin{array}{c}W 2 \\
(\mathrm{mag})\end{array}$ & $\begin{array}{c}\text { W3 } \\
\text { (mag) }\end{array}$ & $\begin{array}{c}\text { W4 } \\
(\mathrm{mag})\end{array}$ & $\begin{array}{l}{[3.6]} \\
\text { (mag) }\end{array}$ & $\begin{array}{l}{[4.5]} \\
(\mathrm{mag})\end{array}$ & $\begin{array}{l}5.8] \\
(\mathrm{mag})\end{array}$ & $\begin{array}{l}{[8.0]} \\
(\mathrm{mag})\end{array}$ & $\begin{array}{c}{[24]} \\
(\mathrm{mag})\end{array}$ & $\begin{array}{c}\alpha \\
2-8 \mu \mathrm{m}\end{array}$ & $\begin{array}{c}\alpha \\
2-24 \mu \mathrm{m}\end{array}$ & $\begin{array}{c}\alpha \\
3.6-8 \mu \mathrm{m}\end{array}$ & $\begin{array}{c}\alpha \\
3.6-24 \mu \mathrm{m}\end{array}$ & Class \\
\hline 1 & 053605.00 & -064243.8 & $10.87 \pm 0.02$ & $10.37 \pm 0.02$ & $8.55 \pm 0.14$ & $6.17 \pm 0.18$ & $10.65 \pm 0.06$ & $10.29 \pm 0.06$ & $10.06 \pm 0.06$ & $9.50 \pm 0.07$ & $6.88 \pm 0.08$ & -1.49 & -1.16 & -1.55 & -1.07 & II \\
\hline 2 & 053510.69 & -063415.5 & $11.73 \pm 0.02$ & $11.18 \pm 0.02$ & $\ldots$ & $\ldots$ & $11.93 \pm 0.06$ & $11.41 \pm 0.06$ & $11.28 \pm 0.06$ & $10.93 \pm 0.09$ & $8.52 \pm 0.22$ & -1.68 & -1.38 & -1.77 & -1.30 & II \\
\hline 3 & 053452.29 & -063508.5 & $12.03 \pm 0.03$ & $11.85 \pm 0.02$ & $\ldots$ & $\ldots$ & $12.09 \pm 0.06$ & $11.99 \pm 0.06$ & $11.94 \pm 0.06$ & $11.86 \pm 0.10$ & $\ldots$ & -2.35 & $\ldots$ & -2.59 & $\ldots$ & III \\
\hline 4 & 053550.56 & -063317.3 & $11.59 \pm 0.03$ & $11.41 \pm 0.03$ & $\ldots$ & $7.21 \pm 0.39$ & $11.51 \pm 0.06$ & $11.47 \pm 0.06$ & $11.40 \pm 0.07$ & $11.40 \pm 0.17$ & $\ldots$ & -2.50 & $\ldots$ & -2.70 & $\ldots$ & III \\
\hline 5 & 053510.75 & -063444.5 & $10.10 \pm 0.02$ & $10.12 \pm 0.02$ & $\ldots$ & $\ldots$ & $10.07 \pm 0.06$ & $10.03 \pm 0.06$ & $10.03 \pm 0.06$ & $10.13 \pm 0.07$ & $\ldots$ & -2.72 & $\ldots$ & -2.92 & $\ldots$ & III \\
\hline
\end{tabular}

(This table is available in its entirety in a machine-readable form in the online journal. A portion is shown here for guidance regarding its form and content.) 

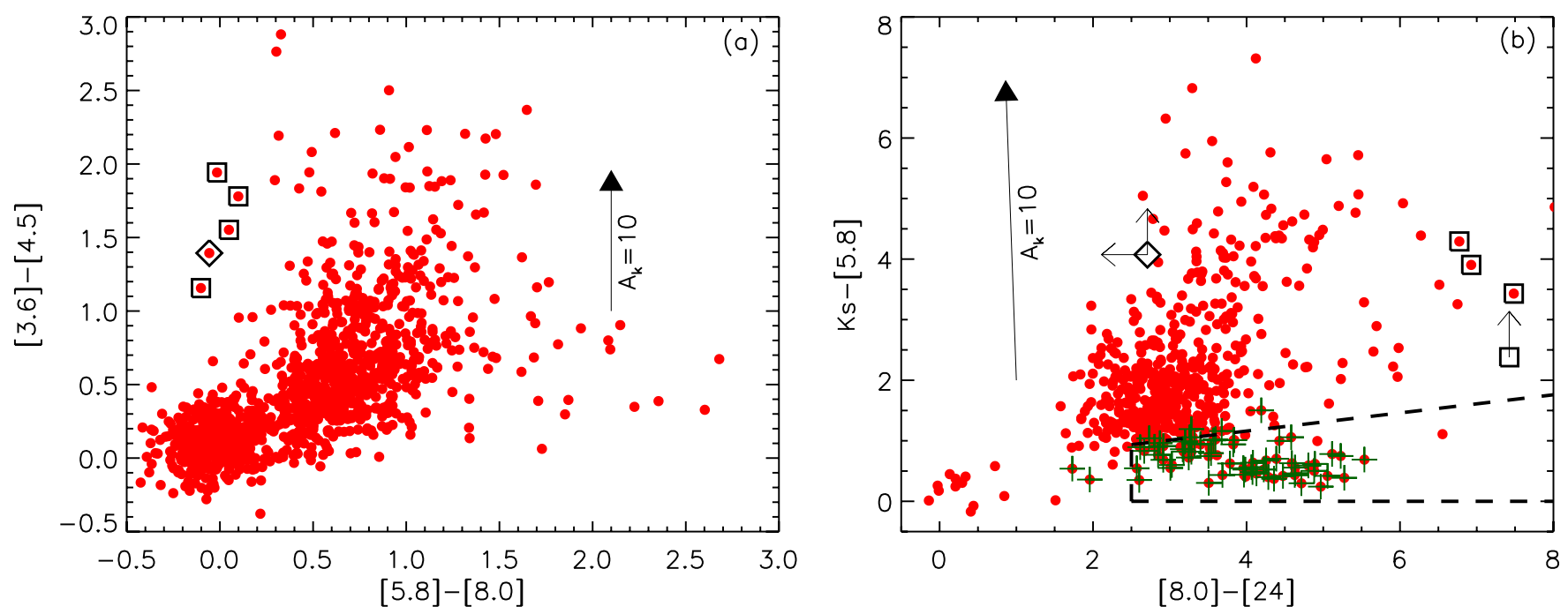

Figure 6. (a) [3.6] - [4.5] vs. [5.8] - [8.0] color-color diagram. The open diamond marks source 1074, and the open boxes show sources ID 1218, 1220, 1233, and 1244. These five sources show [5.8] - [8.0] colors close to zero, but [3.6] - [4.5] > 1.0. (b) [8.0] - [24] vs. $K_{\mathrm{S}}-$ [5.8] color-color diagram. The dashed lines enclose the region for selecting TDs. The pluses mark the TDs in Paper I and this work. The large arrow in each panel shows the reddening vector with a $K_{\mathrm{s}}$-band extinction of 10 mag. The open diamond and boxes are the same as in panel (a). The small arrows close to the open symbols mark the upper or lower limits for the colors of the sources.

(A color version of this figure is available in the online journal.)

within $K_{\mathrm{s}}-[5.8]>1$, and [8.0] $-[24]>2$. There is a minor group of YSOs with $K_{\mathrm{s}}-[5.8] \lesssim 1$, and [8.0] $-[24] \gtrsim 2.5$, indicating that these sources show very weak or no excess emission in the shorter IRAC bands, but strong excess emission in $24 \mu \mathrm{m}$, characteristic of TDs (see Paper I). The five unusual sources in Figure 6(a) are also marked in Figure 6(b). Among them, two sources (1074 and 1233) are not detected in either the 2MASS survey or the UKIDSS survey, and an upper $K_{\mathrm{s}}$-band limiting magnitude of 15.3 is assumed for these sources. Source 1074 is not detected in the $24 \mu \mathrm{m}$ band, and its upper limiting magnitude at $24 \mu \mathrm{m}$ is used for the plot.

\subsubsection{The Completeness and Contamination of the YSO Census}

The data sets used in our YSO census consist of different surveys, each of which has slightly different sky coverage (see Figure 2) and different sensitivities for detecting YSOs. The $X M M$ survey is more sensitive to Class III sources than Class II sources (Feigelson \& Montmerle 1999) and is insensitive to the intermediate-mass stars (Feigelson et al. 2003). The Spitzer imaging survey can be used to identify YSOs with circumstellar disks. The spectroscopic surveys can be used to confirm the youth properties of stars, but are limited by extinction. Thus, it is difficult to quantify the completeness of our YSO census. Here, we only qualitatively evaluate it based on the $K$-band luminosity function (KLF) of our YSO sample.

The shapes of KLFs of young clusters are sensitive to their initial mass functions (IMFs) and mean ages (Muench et al. 2000), and thus can be used to constrain these properties (Strom et al. 1993; Lada \& Lada 1995; Muench et al. 2002, 2003). In our YSO catalog, $84 \%$ of the YSOs have been detected in the 2MASS survey. In Figure 7(a), we show the KLF of these YSOs. The KLF of L1641 peaks at $\sim 12$ mag (see Figure 7(a)), consistent with the KLFs of $\sim 1 \mathrm{Myr}$ old clusters (Muench et al. 2000). As a comparison we also show the KLF (with the $K$-band completeness limit of $\sim 17.5 \mathrm{mag}$ ) of the Trapezium cluster which is at an age ( $\sim 1 \mathrm{Myr})$ similar to L1641 (Muench et al. 2002; Hillenbrand 1997). With the assumption that L1641 and Trapezium have a similar IMF, one would expect them to

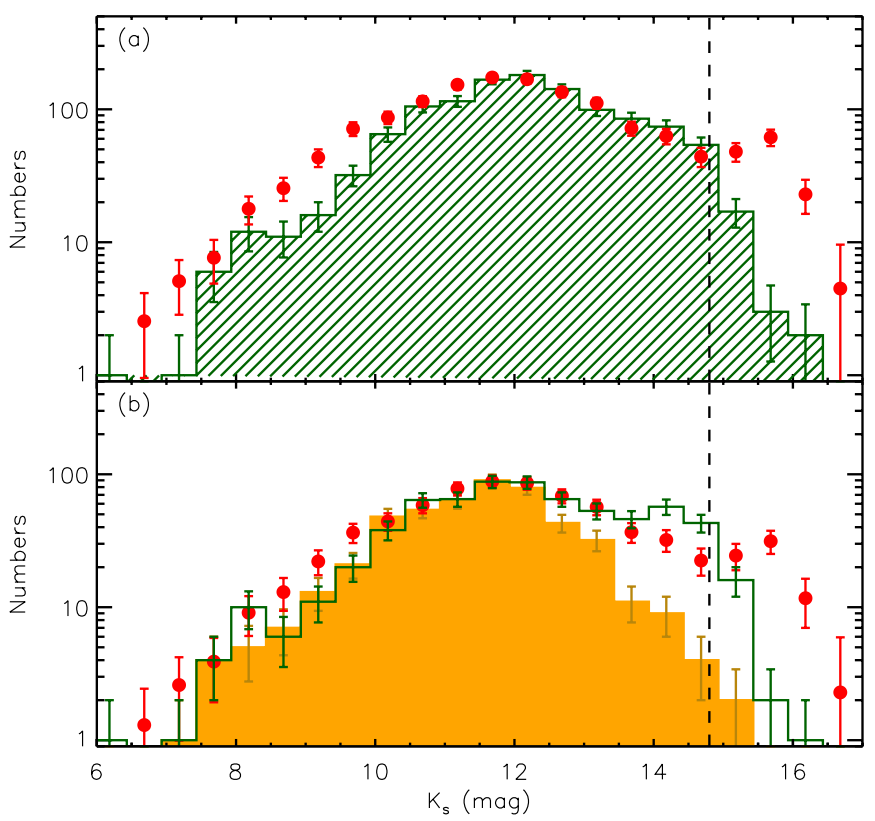

Figure 7. (a) KLFs for all YSOs in L1641 (hatched histograms) and in the Trapezium cluster (filled circles). The Trapezium KLF from Muench et al. (2002) has been shifted from $414 \mathrm{pc}$ (Menten et al. 2007) to $450 \mathrm{pc}$, and scaled to match the peak of the KLF of L1641. The dashed line marks the completeness limit of the 2MASS survey. (b) KLFs for YSOs with infrared excess (open histograms) and for YSOs with X-ray emission (filled histograms) in L1641. The scaled KLF in the Trapezium cluster (filled circles) is also shown for comparison.

(A color version of this figure is available in the online journal.)

show a similar KLF shape. Thus, the comparison of the KLFs of L1641 and Trapezium can be used to qualitatively evaluate the completeness of our YSO census in L1641.

In general, the KLF of L1641 (see Figure 7(a)) fits that of the Trapezium cluster within the range of $10-15 \mathrm{mag}$, corresponding to a mass range of $1.0-0.04 M_{\odot}$ for 1 Myr PMS stars (Dotter et al. 2008; Baraffe et al. 1998). However, at the faint ( $215 \mathrm{mag})$ 
and bright (8-10 mag) ends of the KLF, L1641 appears to be deficient in stars, compared with the Trapezium cluster. The lack of faint YSOs in L1641 is mainly attributed to the incompleteness of the 2MASS survey. In our YSO catalog, $16 \%$ of the YSOs are not detected by the 2MASS survey. These objects can partially contribute to the faint part of the KLF of L1641. Within the range of $8-10 \mathrm{mag}$, corresponding to intermediate-mass 1 Myr PMS stars (Dotter et al. 2008), there is another deficiency in YSOs, which could be attributed to the fact that intermediate-mass stars usually show very weak $\mathrm{X}$-ray emission and would not be picked up by the XMM survey (Feigelson et al. 2003). These intermediate-mass YSOs can be detected in the optical and infrared imaging surveys, but if they have lost their circumstellar disks, then they cannot be identified as YSOs according to their infrared colors. The comparison of the KLFs of L1641 and Trapezium suggests our YSO census may lack $\sim 60$ intermediate-mass diskless stars in L1641. Recently, Hsu et al. (2012, 2013) have proposed that L1641 and the Trapezium cluster may have different IMFs, and L1641 is deficient in the stars at the upper mass end of the IMF, compared with that of the Trapezium cluster. Thus, the actual number of missing intermediate-mass diskless stars in our YSO census may be less than what we expected.

In Figure 7(b), we show the KLFs of two populations of YSOs in our sample: one for the young stars with infrared excess and the other for those with X-ray emission. It can be noted that both KLFs are similar when $K_{\mathrm{s}}<12.5 \mathrm{mag}$, corresponding to 1 Myr PMS stars with masses $\gtrsim 0.2 M_{\odot}$, though both populations share only the 159 stars with $K_{\mathrm{s}}<12.5 \mathrm{mag}$. At the faint end of the KLF $\left(K_{\mathrm{s}}>12.5 \mathrm{mag}\right)$, X-ray-emitting YSOs are clearly deficient in stars compared with YSOs with infrared excess, which could be due to the incompleteness of the $X M M$ survey. We evaluate the completeness of the $X M M$ observations in L1641 using the tool Flux Limits from Images from XMMNewton (FLIX), ${ }^{7}$ which can be used to roughly estimate the upper limit from the observed $X M M$ image. We used the FLIX tool to estimate the $5 \sigma$ upper limit of the $0.2-2 \mathrm{keV}$ $\mathrm{X}$-ray luminosity in the field of L1641. We found that the $5 \sigma$ upper limits vary from region to region with a typical value $\sim 2.4 \times 10^{29} \mathrm{erg} \mathrm{s}^{-1}$ at a distance $(\sim 450 \mathrm{pc})$ of L1641. According to the relation between the X-ray luminosity and stellar mass for the young stars in Taurus $(\sim 1.5 \mathrm{Myr})$ from Güdel et al. (2007), the typical $5 \sigma$ upper limit of the X-ray observations corresponds to the X-ray luminosity of 1.5 Myr old PMS stars with masses of $0.2-0.3 M_{\odot}$, which is consistent with the estimate of incompleteness from the KLF of X-ray sources. In the FOVs of the XMM observations, we have identified 395 Class III sources with spectroscopy. Among these, 253 sources are brighter than $12.5 \mathrm{mag}$ in the $K_{\mathrm{s}}$ band, and $\sim 78 \%(197 / 253)$ of them show X-ray emission. The fraction of X-ray-emitting sources among the faint Class III sources $\left(K_{\mathrm{s}}>12.5 \mathrm{mag}\right)$ decreases to $\sim 38 \%(54 / 142)$. On the one hand, these statistics confirm the results on the completeness of the YSOs selected from the X-ray data estimated using FLIX and KLF; on the other hand, they indicate that the spectroscopic surveys have partially relieved the incompleteness of Class III sources suffered in the $\mathrm{X}$-ray survey.

\footnotetext{
7 FLIX is an online tool provided by the XMM-Newton Survey Science Center (see http://www.ledas.ac.uk/flix/flix.html). It provides robust estimates of the X-ray upper limit to a given point in the sky where there are no sources detected in the 2XMMi catalog. See Carrera et al. (2007) for a detailed discussion of the upper limit algorithm.
}

We estimate the approximate completeness of our YSO census based on the study of Hsu et al. (2012) in which the targets for the spectroscopic survey are selected from the $V$ versus $V-I$ color-magnitude diagram. The spectroscopic survey of Hsu et al. (2012) is complete to $V \lesssim 21$ mag for the disk population, and has $\sim 95 \%$ completeness for the diskless population up to $V \lesssim 21 \mathrm{mag}$, which corresponds to a $1.5 \mathrm{Myr}$ old, $\sim 0.2 M_{\odot}$ PMS star with $A_{\mathrm{V}} \sim 3$. The faint disk population sources ( $V \gtrsim 21 \mathrm{mag}$ ), most of which are heavily extincted objects, are below the detection limit, but can easily be detected in the Spitzer imaging survey. In our catalog, there are 533 Class II sources. Among these, 330 are in Hsu et al. (2012). The other 203 Class II sources are too faint to be observed. In the same field, Hsu et al. (2012) have found 388 Class III sources. If we assume that both Class II and Class III sources have a similar fraction of faint sources, which are unobservable in the spectroscopic survey of Hsu et al. (2012), then the total number of Class III sources is expected to be $\sim 660$. In our YSO catalog, 507 Class III sources are inventoried. Thus, the completeness of our YSO census is estimated to be $\sim 90 \%(1388 / 1541)$. In addition, we can evaluate the completeness of our YSO sample using the $r$ versus $r-i$ color-magnitude diagram (see the detailed discussion in Appendix C). Doing this, we find that our census is complete to $r \lesssim 20$ which corresponds to a $1.5 \mathrm{Myr}$ old, $0.2 M_{\odot}$ PMS star with an extinction of $A_{\mathrm{V}} \sim 3$.

Our YSO catalog may be contaminated by a small fraction of extragalactic sources and nearby foreground stars. These contaminants show SEDs similar to those of YSOs or X-ray emission, thus they are selected as YSO candidates. The main extragalactic contaminants include star-forming galaxies and AGNs (Gutermuth et al. 2008). According to the criteria of Gutermuth et al. (2008), which are used to identify potential extragalactic contaminants based on the IRAC photometry and colors, 92 out of 1273 sources with detection in four IRAC bands are suggested to be contaminants. Among the 92 "contaminants," 13 are observed with spectroscopy and confirmed to be young stars, thus they excluded from the contaminants. Among the remaining 79 sources without spectroscopy, 66 "contaminants" show SEDs with a shape characteristic of Class I/flat YSOs, and 13 are classified as Class II sources. Thus, statistically, about $6 \%(79 / 1273)$ of our YSO candidates could be extragalactic contaminants. This, however, is a conservative estimate: a few of these "contaminants" are located in the dense regions of the L1641 cloud, and thus they could be real YSOs. Foreground stars can also contaminate our YSO catalog. These stars show detectable X-ray emission, thus leading to a wrong classification as Class III sources. The typical X-ray luminosity of field mainsequence stars is several $\times 10^{27} \mathrm{erg} \mathrm{s}^{-1}$ for solar-type to M-type main-sequence stars (Güdel 2004). According to the $5 \sigma$ upper limit of the $0.2-2 \mathrm{keV} X$-ray luminosity in the $X M M$ survey of L1641 estimated with FLIX, XMM observations can detect field stars within the distance $\lesssim 90 \mathrm{pc}$. Using the Besançon model of stellar population synthesis of the Galaxy (Robin et al. 2003), we obtain a small number $(\sim 15)$ of stars within the distance $\lesssim 90 \mathrm{pc}$ in the direction of L1641, suggesting that the fraction of contaminants from foreground stars in our YSO catalog is about $1 \%$.

Recently, Alves \& Bouy (2012) proposed that there is a large cluster, NGC 1980, in front of the Orion A cloud. They separated foreground stars and young stars in the Orion A region based on an assumption that young stars in Orion A are reddened and foreground stars have negligible amount of extinction. They found that the foreground sources are mainly 

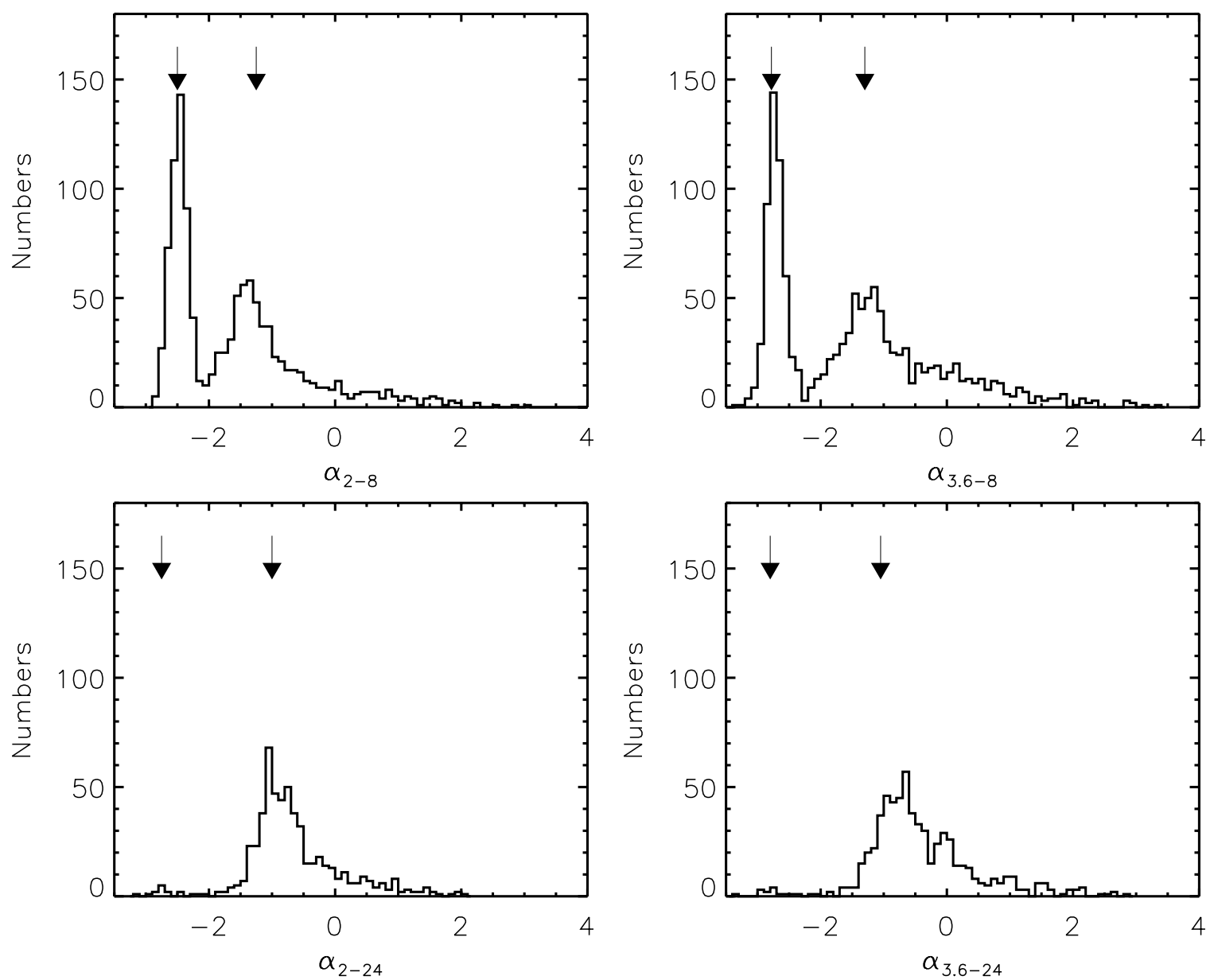

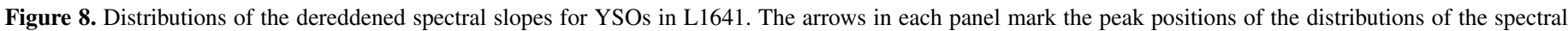
slopes for YSOs in Taurus (Luhman et al. 2010).

centered on NGC 1980. The extent of their suggested foreground population reaches the northern part of L1641, and therefore could contaminate our YSO sample in L1641. The age of the NGC 1980 cluster is estimated to be $\sim 4-5 \mathrm{Myr}$, which is much older than the median age $(\sim 1 \mathrm{Myr})$ of the stellar population in our L1641 sample (Fang et al. 2009; this work). The kinematics of the stellar population in NGC 1980 is similar to that of young stars and molecular gas in Orion A, suggesting that NGC 1980 may be physically associated with Orion A. It is possible that our sample could be contaminated with the older population of NGC 1980, which could result in an older median age and lower disk fraction. We inspect the possible contamination of our YSO census by these "old" sources by comparing the ages of sources with $A_{\mathrm{V}}<0.1$ and those with $A_{\mathrm{V}}>0.5$. The median ages of the two samples are 1.6 Myr and 1.3 Myr, respectively. Thus, we expect that the contamination in our YSO sample is not significant.

\subsubsection{Infrared Spectral Slopes}

Figure 8 shows the distributions of the dereddened spectral infrared slopes, $\alpha_{2-8}, \alpha_{3.6-8}, \alpha_{2-24}$, and $\alpha_{3.6-24}$, for the YSOs in L1641. The disked and diskless objects appear in two distinct peaks in four panels in Figure 8, although only a few diskless YSOs are detected at $24 \mu \mathrm{m}$. As a comparison, in Figure 8, we also show the peak positions of such distributions for YSOs in Taurus, which has an age similar to that of L1641. Both star-forming regions show similar peak positions for the disk population, suggesting that the disks are at a similar evolutionary stage.

Using the spectral slopes $\alpha_{3.6-8}$ and $\alpha_{3.6-24}$, we classify our sources into Class III, Class II, flat-spectrum, and Class I sources and list their types in Table 3 according to the criteria described in Section 3.4.1. We have 507 Class III sources, 533 Class II sources, 131 flat-spectrum sources, and 143 Class I sources. In Figure 2, we show their spatial distributions. The ratios of Class I to Class II sources and flat-spectrum to Class II sources are 0.27 and 0.25 , respectively, comparable to those $(\sim 0.27$ and $\sim 0.20$, respectively) in the Spitzer c $2 \mathrm{~d}$ Legacy survey.

\subsection{Spectroscopic Sample}

We have obtained optical spectra for a large sample of the YSOs in L1641 with Hectospec and Hectochelle. In this section, we will present the results from these observations.

\subsubsection{Spectral Types}

We determined the spectral type of each star in our spectroscopic sample as described in Section 3.2. We have $\sim 400$ stars with reliable estimates of their spectral types. Among them, $\sim 300$ sources are identified as young stars according to the criteria in Section 3.1, and others as field stars. The spectral types of the YSOs are listed in Table 4. In Figure 9(a), we show a histogram of spectral types for all stars, including field stars and YSOs, in our Hectospec survey. The distribution is bimodal, with peaks around spectral type G0 and mid-M. In this 
Table 4

Stellar and Disk Properties of the YSOs in L1641

\begin{tabular}{|c|c|c|c|c|c|c|c|c|c|c|c|}
\hline & $\begin{array}{c}\text { R.A. } \\
(\text { J2000) }\end{array}$ & $\begin{array}{c}\text { Decl. } \\
(\mathrm{J} 2000)\end{array}$ & Spt & $\begin{array}{l}\text { Lum } \\
\left(L_{\odot}\right)\end{array}$ & $\begin{array}{c}A_{\mathrm{V}} \\
(\mathrm{mag})\end{array}$ & $\begin{array}{c}\mathrm{EW}_{\mathrm{H} \alpha} \\
(\AA)\end{array}$ & $\begin{array}{c}\mathrm{EW}_{\mathrm{H} \beta} \\
(\AA)\end{array}$ & $\begin{array}{c}\log \dot{M}_{\mathrm{acc}}^{\mathrm{H} \alpha} \\
\left(M_{\odot} \mathrm{yr}^{-1}\right)\end{array}$ & $\begin{array}{c}\log \dot{M}_{\mathrm{acc}}^{\mathrm{H} \beta} \\
\left(M_{\odot} \mathrm{yr}^{-1}\right)\end{array}$ & $\mathrm{TTS}^{\mathrm{a}}$ & Disk $^{b}$ \\
\hline 1 & 053605.00 & -064243.8 & M5.5 & 0.398 & 0.0 & -23.6 & -14.9 & -9.1 & -9.6 & $\mathrm{C}$ & Thick \\
\hline 2 & 053510.69 & -063415.5 & M4.5 & 0.090 & 0.0 & -36.3 & -24.2 & -9.9 & -10.3 & $\mathrm{C}$ & Thick \\
\hline 3 & 053452.29 & -063508.5 & M4 & 0.128 & 0.0 & -8.4 & -8.2 & $\ldots$ & $\ldots$ & W & $\mathrm{N}$ \\
\hline 4 & 053550.56 & -063317.3 & M3 & 0.254 & 0.0 & -3.8 & -3.5 & $\ldots$ & $\ldots$ & W & $\mathrm{N}$ \\
\hline 5 & 053510.75 & -063444.5 & F5.5 & 4.774 & 0.3 & 1.7 & 2.5 & $\ldots$ & $\ldots$ & W & $\mathrm{N}$ \\
\hline
\end{tabular}

Notes.

a The types of T Tauri stars: C for CTTSs and W for WTTSs.

b The types of disks: $\mathrm{N}$ for diskless stars, Thick for the optically thick disks $\left(\alpha_{3.6-8} \geqslant-1.8\right)$, Thin for the optically thin disks $\left(-2.5 \lesssim \alpha_{3.6-8}<-1.8\right)$, TD for the transition disks, and DD for the debris disk candidates.

(This table is available in its entirety in a machine-readable form in the online journal. A portion is shown here for guidance regarding its form and content.)

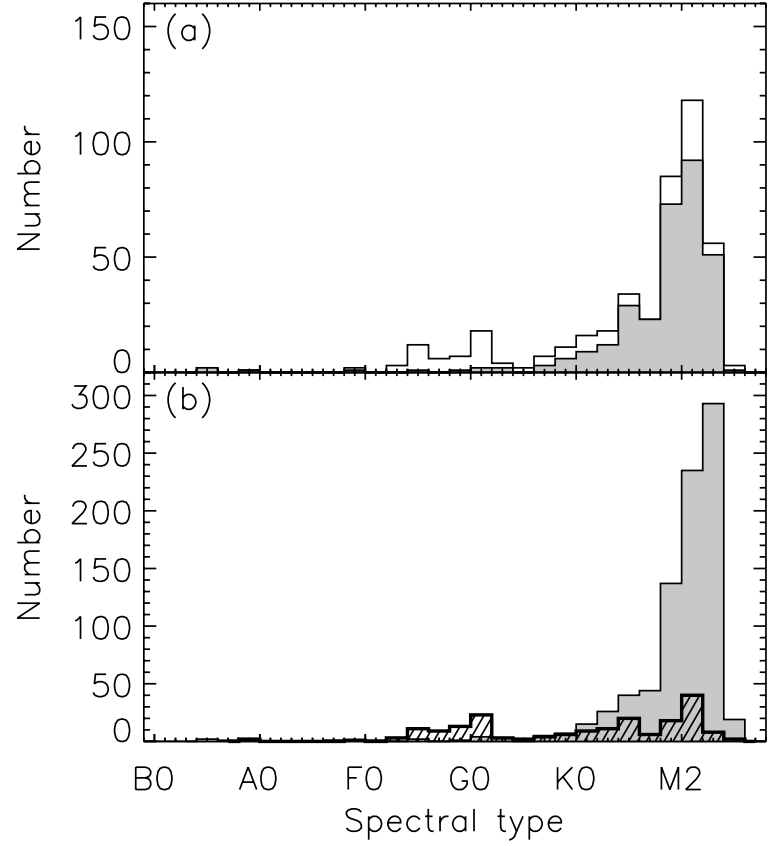

Figure 9. (a) Spectral-type distribution of our sample observed with Hectospec in L1641. The open histograms show the distribution of all the stars with reliable spectral types. The filled histograms display the YSO distribution. (b) The gray shaded histograms show the spectral-type distribution of all known YSOs with spectral types in L1641. The hatched histograms display the spectral-type distributions of field stars identified in both works.

figure, we also show the spectral-type distribution of the identified YSOs. These YSOs are typically K- or M-type stars with few stars earlier than K0-type. We combined the YSOs and field stars with spectral types in the field of L1641, and display their spectral-type distributions in Figure 9(b). The field stars show a bimodal distribution with peaks around spectral type G0 and early-M, while the YSOs peak around early-M.

\subsubsection{Stellar Masses and Ages}

With the determined effective temperatures and bolometric luminosities as described in Section 3.3, we can place the young stars in the H-R diagram. In Figure 10, we show the $\mathrm{H}-\mathrm{R}$ diagrams for the YSOs with spectral types. Most of our YSOs lie between the 0.1 and $3 \mathrm{Myr}$ isochrones. We use distinct symbols (open star symbols) for objects with SEDs typical for TDs (see also Section 4.3.1), as well as a new "exotic" object that is apparently underluminous (see Section 4.5.2). In Table 5, we list the stellar masses and ages of YSOs with

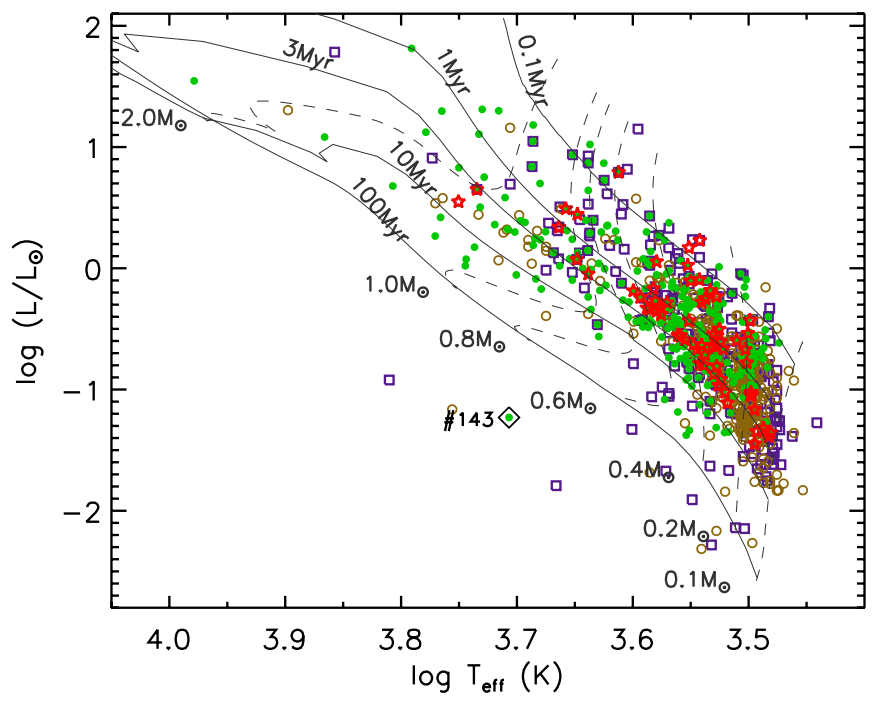

Figure 10. H-R diagram for the YSOs in L1641. The filled circles are for the YSOs in our spectroscopic survey with Hectospec, the open boxes are the YSOs in Paper I, and the open circles are the YSOs in Hsu et al. (2012). The stars represent the TDs in L1641. The open diamond marks the new subluminous object (see Section 4.5.2). The PMS evolutionary tracks are from Dotter et al. (2008).

(A color version of this figure is available in the online journal.)

spectral types from our Hectospec observations, as well as $\sim 300$ YSOs (ID $\geqslant 525$ ) with spectral types from Hsu et al. (2012). In Figure 11(a), we show the mass distribution (filled histograms) for the YSOs in this work, and the mass distribution (open histograms) when adding the YSOs with spectral types in the literature. The median masses are $\sim 0.40 M_{\odot}$ and $0.30 M_{\odot}$ for the two populations, respectively. A linear regression fit to the mass functions within the mass bin $-0.5 \leqslant \log \left(M_{\star} / M_{\odot}\right) \leqslant$ 0.5 yields slopes of $-1.24 \pm 0.13$ and $-1.50 \pm 0.15$ for the two populations, respectively. In Figure 11(b), we show the age distribution of the two sample populations as in Figure 11(a). The median age for the entire sample is $1.5 \mathrm{Myr}$.

Lithium depletion trends in young PMS stars can be used to estimate the stellar ages at sub-solar masses (Mentuch et al. 2008; Jeffries et al. 2009). In Figure 12, we show the EW of the Li I $\lambda 6708$ absorption line as a function of spectral type for the YSOs in our sample. The EW of the Li I $\lambda 6708$ absorption line for each YSO is derived from the Hectochelle spectra. Some YSOs have been observed at multiple epochs with Hectochelle and for these YSOs, we show their mean EWs weighted by the uncertainty in each spectral epoch. 
Table 5

Stellar Masses and Ages of the YSOs

\begin{tabular}{|c|c|c|c|c|c|c|c|c|c|c|c|c|}
\hline & \multirow[b]{2}{*}{ R.A. } & \multirow[b]{2}{*}{ Decl. } & \multicolumn{2}{|c|}{ D08 } & \multicolumn{2}{|c|}{ SO0 } & \multicolumn{2}{|c|}{ B98 } & \multicolumn{2}{|c|}{ DM97 } & \multicolumn{2}{|c|}{ Pisa11 } \\
\hline & & & Mass & Age & Mass & Age & Mass & Age & Mass & Age & Mass & Age \\
\hline ID & $(\mathrm{J} 2000)$ & $\mathrm{J} 2000$ & $\left(M_{\odot}\right)$ & (Myr) & $\left(M_{\odot}\right)$ & (Myr) & $\left(M_{\odot}\right)$ & (Myr) & $\left(M_{\odot}\right)$ & (Myr) & $\left(M_{\odot}\right)$ & (Myr) \\
\hline 1 & 053605.00 & -064243.8 & $0.16_{-0.00}^{+0.00}$ & $0.1_{-0.0}^{+0.0}$ & $0.20_{-0.01}^{+0.01}$ & $0.2_{-0.0}^{+0.0}$ & $\ldots$ & $\ldots$ & $0.13_{-0.00}^{+0.00}$ & $0.1_{-0.0}^{+0.0}$ & $\ldots$ & $\ldots$ \\
\hline 2 & 053510.69 & -063415.5 & $0.20_{-0.01}^{+0.01}$ & $2.0_{-0.3}^{+0.3}$ & $0.18_{-0.01}^{+0.01}$ & $4.1_{-0.1}^{+0.1}$ & $0.21_{-0.01}^{+0.01}$ & $2.5_{-0.3}^{+0.3}$ & $0.18_{-0.01}^{+0.01}$ & $2.1_{-0.1}^{+0.1}$ & $\ldots$ & $\ldots$ \\
\hline 3 & 053452.29 & -063508.5 & $0.23_{-0.04}^{+0.05}$ & $1.6_{-0.5}^{+1.0}$ & $0.22_{-0.04}^{+0.04}$ & $3.4_{-0.5}^{+0.6}$ & $0.24_{-0.04}^{+0.06}$ & $2.2_{-0.5}^{+1.8}$ & $0.19_{-0.02}^{+0.04}$ & $1.6_{-0.2}^{+0.5}$ & $0.22_{-0.01}^{+0.05}$ & $3.6_{-0.1}^{+0.5}$ \\
\hline 4 & 053550.56 & -063317.3 & $0.32_{-0.02}^{+0.03}$ & $1.2_{-0.2}^{+0.3}$ & $0.32_{-0.03}^{+0.02}$ & $2.3_{-0.1}^{+0.2}$ & $0.41_{-0.03}^{+0.04}$ & $2.3_{-0.3}^{+0.5}$ & $0.25_{-0.02}^{+0.03}$ & $1.1_{-0.1}^{+0.1}$ & $0.34_{-0.03}^{+0.03}$ & $2.3_{-0.0}^{+0.2}$ \\
\hline 5 & 053510.75 & -063444.5 & $1.48_{-0.05}^{+0.07}$ & $13.9_{-2.5}^{+3.0}$ & $1.47_{-0.03}^{+0.06}$ & $14.7_{-1.6}^{+5.4}$ & $\ldots$ & $\ldots$ & $1.46_{-0.04}^{+0.07}$ & $13.8_{-2.6}^{+3.6}$ & $1.45_{-0.04}^{+0.06}$ & $13.3_{-1.8}^{+3.5}$ \\
\hline
\end{tabular}

(This table is available in its entirety in a machine-readable form in the online journal. A portion is shown here for guidance regarding its form and content.)
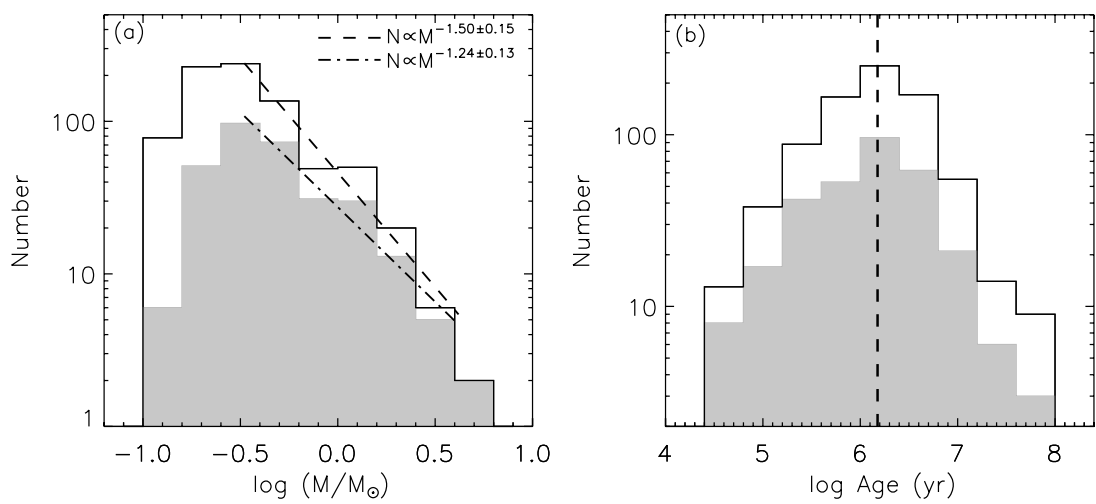

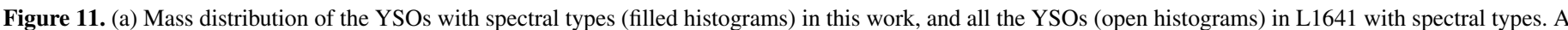

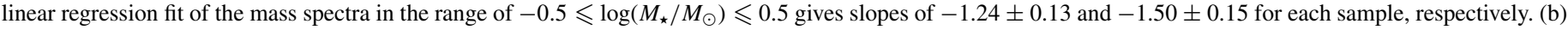

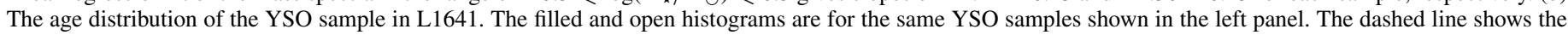
median age for all the YSOs in L1641 with spectral types.

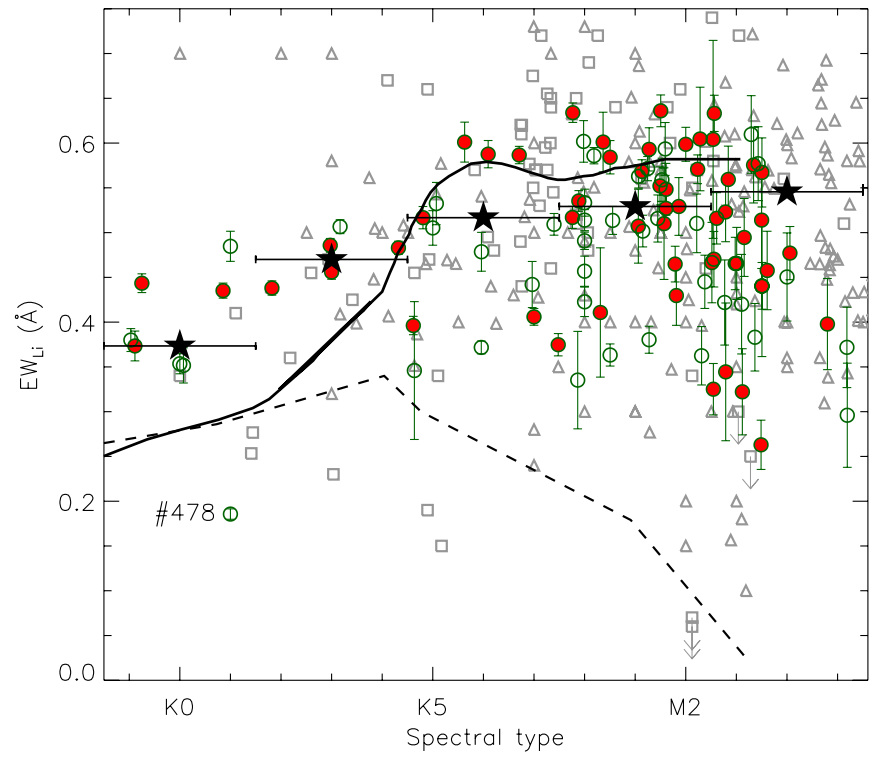

Figure 12. Li I $\lambda 6708 \mathrm{EW}$ vs. spectral type. The open circles and the red filled circles are for the CTTSs and the WTTSs, respectively, in this work. The uncertainties of the EWs are shown with error bars. The open triangles show the YSOs in L1630N and L1641 (Paper I). The open boxes display the YSOs in Taurus (Sestito et al. 2008). The star symbols show the median EW for individual spectral-type bins in the three regions and the bar centered on each star symbol shows the bin size. The solid line shows the model locus for the YSOs with undepleted Li (Baraffe et al. 2002; Jeffries et al. 2009). The dashed line displays the boundary for the Tucanae-Horologium association with an age of $27 \pm 11$ Myr (Mentuch et al. 2008).

(A color version of this figure is available in the online journal.)
In Figure 12, the EWs of CTTSs are generally smaller than those of weak T Tauri stars (WTTSs) with similar spectral types due to the veiling effect in which continuum emission from accretion shocks on the surface of the star can reduce the measured EW of CTTSs. In Figure 12, it can also be noted that the EW of Li I $\lambda 6708$ in WTTSs with similar spectral types shows a large scatter, which may be due to the age spread in these WTTSs. As a comparison, Figure 12 also shows the EWs of Li I $\lambda 6708$ for the YSOs in Taurus (Sestito et al. 2008), and in L1630N and L1641 presented in Paper I. The L1641 starforming region is roughly the same age as Taurus and L1630N, thus it is expected to show a lithium depletion trend similar to those in Taurus and L1630N. Using the EW of the Li I $\lambda 6708$ absorption line for YSOs in the three regions, we estimate the median EW in each spectral-type bin (filled star symbols in Figure 12). For the YSOs with spectral types later than K5, the median EWs generally agree with the theoretical locus for YSOs with undepleted lithium (Baraffe et al. 2002; Jeffries et al. 2009). The median EW and theoretical loci deviate for YSOs with spectral types earlier than K5. The median EWs for the 1 Myr old population are much larger than those in the Tucanae-Horologium stellar association, which has an age of $27 \pm 11$ Myr (Mentuch et al. 2008).

\subsubsection{Extinction}

We estimated the extinction for each individual object using the method outlined in Section 3.3. The typical uncertainty for the extinction values is less than 0.1 mag. In Figure 13(a), we show the $A_{\mathrm{V}}$ distribution of the YSOs (filled histograms) from this work as well as all YSOs (open histograms) with spectral types in L1641. Both populations have a similar $A_{\mathrm{V}}$ distribution 

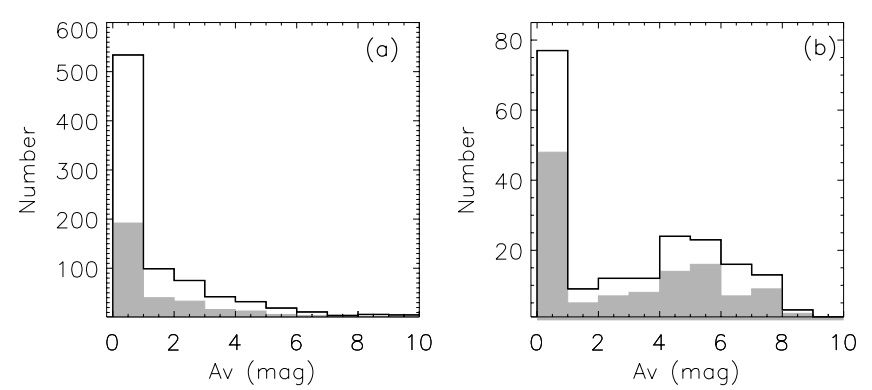

Figure 13. (a) Visual extinction distribution of the YSOs (filled histograms) observed with Hectospec and all known YSOs with spectral types (open histograms). (b) The visual extinction distribution of the non-members in L1641 (filled histograms) identified in this work, and all the non-members (open histograms) in both this work and Paper I.

with a peak at very low extinction $\left(A_{\mathrm{V}}<1 \mathrm{mag}\right)$ and a gradual decrease of YSO numbers toward higher $A_{\mathrm{V}}$. In Figure 13(b), we show the field stars (filled histograms) identified in this work, and all the field stars (open histograms) identified in both this work and Paper I. The two populations of field stars show a similar $A_{\mathrm{V}}$ distribution, but one different from the $A_{\mathrm{V}}$ distribution of the YSO samples (see Figure 13(a)). The $A_{\mathrm{V}}$ distribution of the field stars has a peak at $A_{\mathrm{V}} \lesssim 1$ mag consistent with foreground and background objects along sight lines with low extinction, and a relatively flat distribution between $\sim 1$ and $\sim 8$ mag of $A_{\mathrm{V}}$ with a peak around $A_{\mathrm{V}}=4-6$ mag.

\subsection{Disks}

\subsubsection{Transition Disks}

Our main TD selection criteria are based on colors as shown in Figure 6(b): [8.0] $-[24] \geqslant 2.5$ and $K_{\mathrm{s}}-[5.8] \leqslant$ $0.56+([8.0]-[24]) \times 0.15$. For the YSOs with spectral types, we use the spectral types of the central stars to constrain the photospheric emission. We compare their SEDs with their model photospheric emission to look for the sources showing very weak or no infrared excess at near-infrared wavelengths and shorter IRAC bands but strong excess emission at mid-infrared and longer wavelengths.

In Figure 14, we show the SEDs of the 46 newly confirmed TDs in this work. Among them, 40 TDs are selected based on our

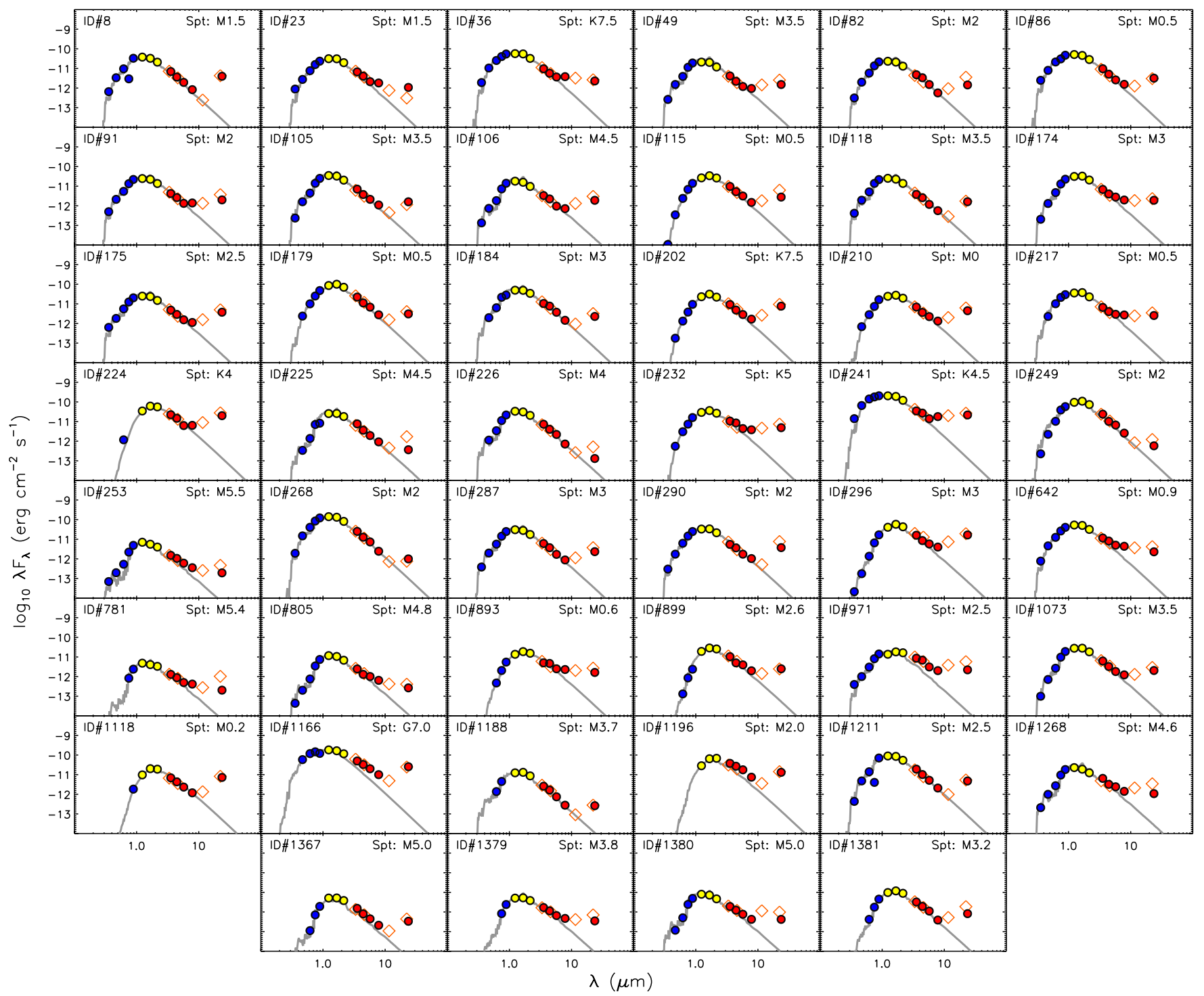

Figure 14. SEDs of newly confirmed YSOs with TDs in L1641. The circles show the photometry from SDSS/LAICA (blue), 2MASS (magenta), and Spitzer (red). The open diamonds are the photometry from WISE. The photospheric emission level is indicated with a gray curve in each panel.

(A color version of this figure is available in the online journal.) 

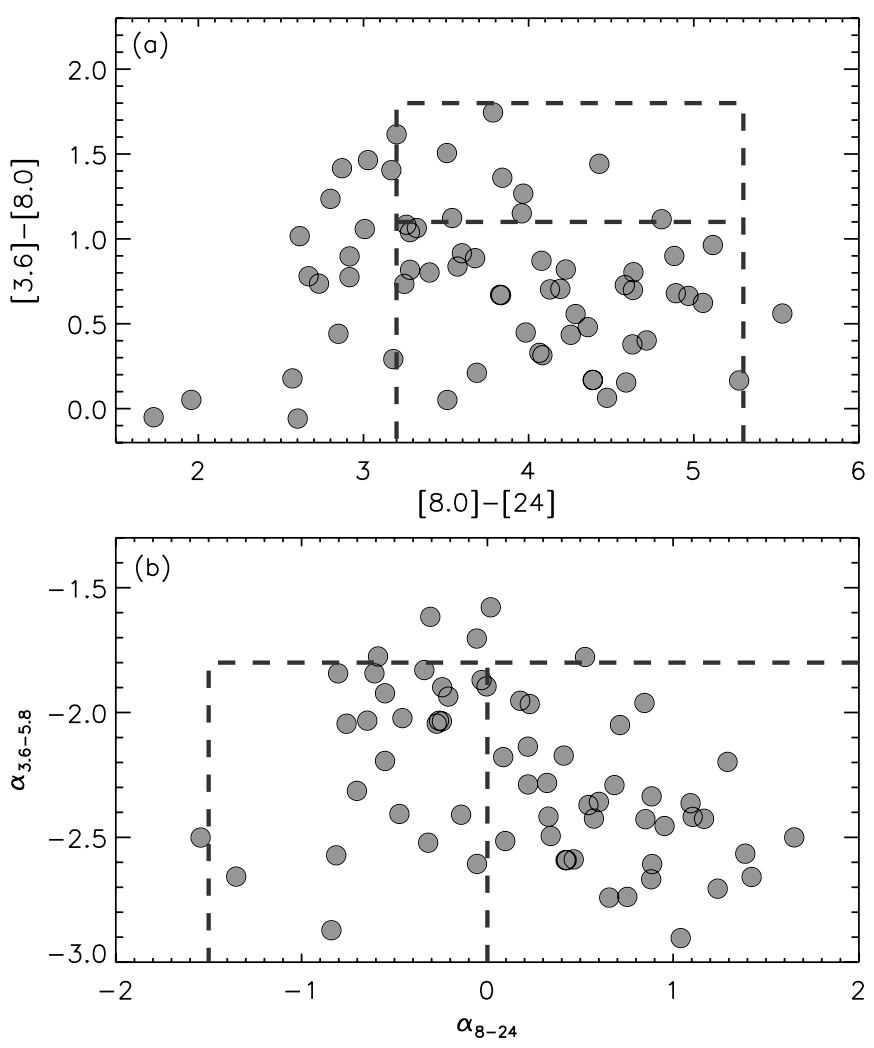

Figure 15. TD selection criteria from (a) Merín et al. (2010) and (b) Muzerolle et al. (2010). The filled circles are our confirmed TDs in L1641 from both Paper I and this work. In panel (a), classical TDs (with inner holes) lie within $0.0<[3.6]-[8.0]<1.1$ and $3.2<[8.0]-[24]<5.3$, and TDs with weak excess emission in Spitzer IRAC bands lie within $1.1<[3.6]-[8.0]<1.8$ and $3.2<[8.0]-[24]<5.3$. In panel (b), the region with $-1.5 \leqslant \alpha_{8-24} \leqslant 0$ and $\alpha_{3.6-5.8} \leqslant-1.8$ is for weak excess TDs, and the region with $\alpha_{8-24}>0$ and $\alpha_{3.6-5.8} \leqslant-1.8$ is for classical TDs.

TD selection criteria (see Figure 6(b)). The additional six TDs are selected by the comparison of their SEDs and photospheric emission. Among the six TDs, two (226 and 249) of them show nearly photospheric emission at wavelengths out to $\sim 8 \mu \mathrm{m}$, and moderate excess at $24 \mu \mathrm{m}([8.0]-[24]<2)$. The other four TDs (971, 1196, 1268, and 1379), showing near-infrared excess emission significantly reduced from normal $\mathrm{T}$ Tauri stars, are located close to the TD selection boundary.

In the literature, there are several criteria to select TDs, e.g., Paper I, Merín et al. (2010), Muzerolle et al. (2010), etc. Our selection criteria, based on $K_{\mathrm{s}}-$ [5.8] and [8.0] - [24] colors, are shown in Figure 6(b). Merín et al. (2010) provide different criteria based on [3.6] - [8.0] and [8.0] - [24] colors: (1) $0.0<$ $[3.6]-[8.0]<1.1$ and $3.2<[8.0]-[24]<5.3$ for classical TDs (with inner holes); (2) $1.1<$ [3.6] $-[8.0]<1.8$ and $3.2<[8.0]-[24]<5.3$ for TDs with weak excess emission in Spitzer IRAC bands. Muzerolle et al. (2010) employ two infrared spectral slopes to select TD candidates: $(1)-1.5 \leqslant \alpha_{8-24} \leqslant 0$ and $\alpha_{3.6-5.8} \leqslant-1.8$ for weak excess TDs; (2) $\alpha_{8-24}>0$ and $\alpha_{3.6-5.8} \leqslant-1.8$ for classical TDs (with inner holes). Our TD sample in this work is mainly selected using the criteria in Paper I. Including the TDs in Paper I, we have 65 TDs with spectral-type estimates in L1641. Among them, 64 TDs are detected in all four IRAC bands and at MIPS $24 \mu \mathrm{m}$. We use these 64 TDs to compare our TD criteria with those from Merín et al. (2010) and Muzerolle et al. (2010). In Figure 15, we show how these TDs fit to the selection criteria from Merín et al. (2010) and Muzerolle et al. (2010). According to the criteria of Merín et al. (2010), 38 sources are classified into their Group (1), and 9 sources are classified into Group (2). Among the remaining 17 sources which are excluded as TDs, 16 objects do not show strong enough excess emission at $24 \mu \mathrm{m}$, and 1 object shows excess emission at $24 \mu \mathrm{m}$ that is too strong (see Figure 15(a)). These rejected sources fall within the criteria of Muzerolle et al. (2010). According to their criteria, 23 sources are categorized into their Group (1), 35 into their Group (2), and 6 sources are excluded as TDs but very close to the selection boundaries. Thus, our selection criteria are generally consistent with those from Merín et al. (2010) and Muzerolle et al. (2010).

\subsubsection{Globally Depleted Disks}

In Figure 16, we show the SEDs of the globally depleted disk candidates in L1641, as well as the median SED of T Tauri stars in Taurus (Furlan et al. 2006). Compared with the median SED of Taurus, these YSOs in Figure 16 show uniformly depleted SEDs with an excess infrared emission level that has been reduced by a similar factor across 3.6-24 $\mu \mathrm{m}$. Such SED types can be produced when disks are deficient in small dust grains (Currie et al. 2009; Sicilia-Aguilar et al. 2011). For these disks, their effective disk height, i.e., the height above the disk midplane where the disk becomes optically thick to the stellar radiation, can be substantially reduced, causing a smaller fraction of the stellar energy to be absorbed and reprocessed by the disk, which produces an infrared excess emission of reduced magnitude. Dust coagulation can lead to a deficiency of small dust grains, and the larger grains couple less efficiently with the gas, allowing them to settle toward the midplane.

\subsubsection{Young Debris Disks or "Evolved" TDs?}

Figure 17 shows the SEDs of two objects 172 and 269. These two sources show essentially photospheric emission levels at $\lambda \lesssim 10 \mu \mathrm{m}$, but display weak excesses at $24 \mu \mathrm{m}$. This SED shape is typical of "debris disks" (see, e.g., Carpenter et al. 2009). Such a disk system possesses relatively small amounts of circumstellar material which are produced in the collisional grind-down of larger bodies ("planetesimals"). In Figure 17, both of the stars have the spectral type K6, and show X-ray emission (see Table 2). Their ages, estimated from different sets of PMS evolutionary tracks, are all less than $1 \mathrm{Myr}$ (see Table 5). The youth of stars 172 and 269 can be further confirmed by our spectroscopic observation. Star 172 has been observed with both Hectochelle and Hectospec and both spectra clearly show the Li I $\lambda 6708$ absorption line. In its high-resolution Hectochelle spectrum, the EW of the Li I $\lambda 6708$ absorption line is $0.53 \AA$. The star 269 has only been observed with Hectospec. In its Hectospec spectrum, the EW of the Li I $\lambda 6708$ absorption line is $\sim 0.5 \AA$. The Li EWs of both stars suggest they are as young as other YSOs in L1641 (see Figure 12). Furthermore, star 172 has a radial velocity $V_{\mathrm{LSR}}=5 \pm 2 \mathrm{~km} \mathrm{~s}^{-1}$ (relative to the local standard of rest) similar to the local molecular gas in L1641 (Bally et al. 1987), suggesting that star 172 is physically associated with the L1641 cloud.

In a case where the disks are optically thin through $24 \mu \mathrm{m}$, we can estimate the timescale for small dust grains removed by Poynting-Robertson drag using the method described in Currie et al. (2009). For $10 \mu \mathrm{m}$ dust grains with a volume density $\left(\rho_{\mathrm{s}}\right) \sim 1 \mathrm{~g} \mathrm{~cm}^{-3}$ and an absorption coefficient $\left(\left\langle Q_{\text {abs }}\right\rangle\right) \sim 1$, the Poynting-Robertson drag can remove these grains on timescales $\lesssim 6 \times 10^{5}$ yr. When the dust grains are porous, the drag time 

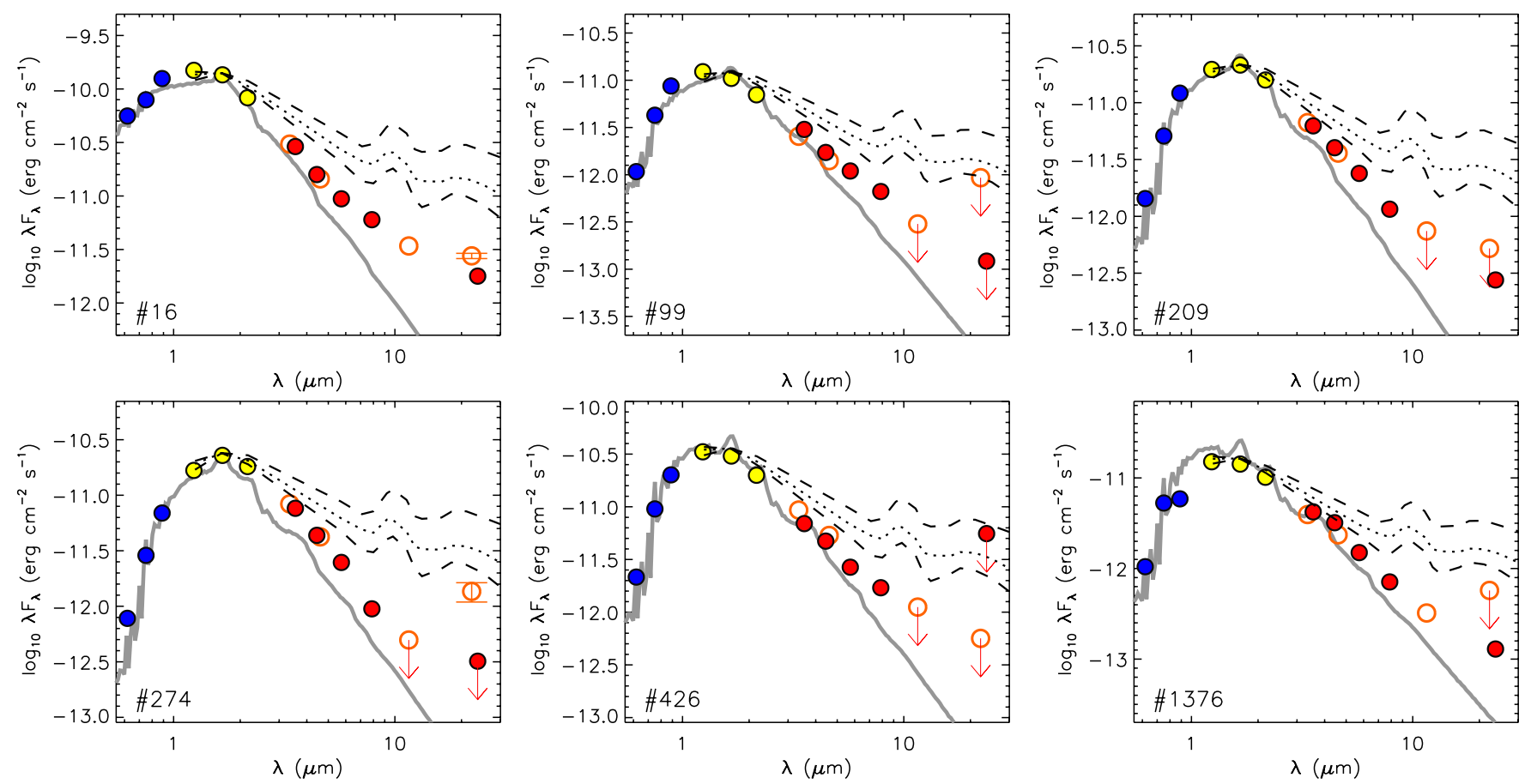

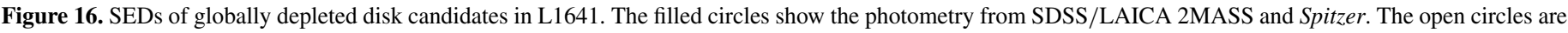

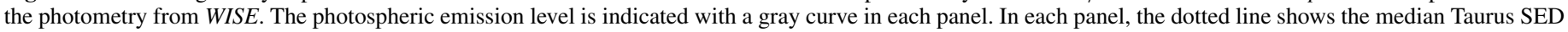
with upper and lower quartiles shown with dashed lines (Furlan et al. 2006).

(A color version of this figure is available in the online journal.)

could be much shorter (Currie et al. 2009). For the two stars, the typical grain sizes, below which the radiation pressure can remove dust from the system, are estimated to be $\sim 3-8 \mu \mathrm{m}$ using Equation (4) in Currie et al. (2009) with $\rho_{\mathrm{s}} \sim 1 \mathrm{~g} \mathrm{~cm}^{-3}$, and $\left\langle Q_{\text {abs }}\right\rangle \sim 1$. However, whether or not the Poynting-Robertson drag can work on dust grains also depends on the coupling of dust to the gas. If the gas density in circumstellar disks is low, then the dust grains emitting excess $24 \mu \mathrm{m}$ emission can be quickly removed on the above dynamical timescale without replenishment from collisions with larger planetesimals. Thus, the disks around the two stars 172 and 269 could be debris if they are at ages of several megayears. However, the ages of stars 172 and 269, estimated from different sets of PMS evolutionary tracks, are all younger than several $10^{5} \mathrm{yr}$. If their isochrone ages are reasonable, then it is also possible that the two sources are relatively evolved TDs with much larger inner holes (several tens of $\mathrm{AU}$ ) in the disks than normal TDs. Such evolved disks could still be gas rich and only produce weak excess emission at $24 \mu \mathrm{m}$.

\subsection{Accretion}

The Balmer emission lines in the spectra of PMS stars can be produced in two ways: chromospheric activity and magnetospheric accretion. The former mechanism usually yields weak and narrow emission lines, while the magnetospheric accretion process can produce very broad and strong emission lines (Hartmann et al. 1994; Muzerolle et al. 2001; White \& Basri 2003). Thus, the strength and width of the Balmer emission lines, especially for the $\mathrm{H} \alpha$ line, are used to characterize the accretion activity of young stars. Our Hectospec spectra cover both the $\mathrm{H} \alpha$ and $\mathrm{H} \beta$ lines with a medium spectral resolution, and the Hectochelle spectra cover the $\mathrm{H} \alpha$ line with a high spectral resolution. We will use all these data to study the accretion properties of young stars.

\subsubsection{Characterizing Accretion Activity with Medium-resolution Spectroscopy}

We classified the YSOs into WTTSs or CTTSs based on their $\mathrm{H} \alpha \mathrm{EW}$ in the Hectospec spectra using the spectral-typedependent criteria described in Paper I. A star is considered to be a CTTS if $\mathrm{EW}(\mathrm{H} \alpha) \geqslant 3 \AA$ for $\mathrm{K} 0-\mathrm{K} 3$ stars, $\mathrm{EW}(\mathrm{H} \alpha) \geqslant 5 \AA$ for $\mathrm{K} 4$ stars, $\mathrm{EW}(\mathrm{H} \alpha) \geqslant 7 \AA$ for $\mathrm{K} 5-\mathrm{K} 7$ stars, $\mathrm{EW}(\mathrm{H} \alpha) \geqslant 9 \AA$ for M0-M1 stars, $\mathrm{EW}(\mathrm{H} \alpha) \geqslant 11 \AA$ for $\mathrm{M} 2$ stars, $\mathrm{EW}(\mathrm{H} \alpha) \geqslant 15 \AA$ for M3-M4 stars, $\mathrm{EW}(\mathrm{H} \alpha) \geqslant 18 \AA$ for M5-M6 stars, and $\mathrm{EW}(\mathrm{H} \alpha) \geqslant 20 \AA$ for M7-M8 stars. These criteria are based on the $\mathrm{H} \alpha$ line EWs, which make them useful for spectra with low spectral resolution. The disadvantage of this definition is that it may misclassify some accretors as WTTSs. In our spectroscopic sample observed with Hectospec, a few of the YSOs (e.g., sources 23 and 36) have been observed with Hectochelle and show broad $\mathrm{H} \alpha$ line profiles, indicating ongoing accreting activities despite the small $\mathrm{H} \alpha \mathrm{EW}$. The $\mathrm{H} \alpha$ line EWs of these sources are below the thresholds for classification as CTTSs because of the strong absorption around their $\mathrm{H} \alpha$ line centers. In this case, we also use the $\mathrm{FW}_{\mathrm{H} \alpha, 10 \%}$ of the Hectochelle spectra to distinguish the CTTSs and WTTSs. As discussed in Appendix A, a YSO can be classified as a CTTS if its $\mathrm{FW}_{\mathrm{H} \alpha, 10 \%}>250 \mathrm{~km} \mathrm{~s}^{-1}$.

In Table 4, we list the EWs of $\mathrm{H} \alpha$ and $\mathrm{H} \beta$ from the Hectospec spectra for our YSO sample, as well as $\sim 300$ YSOs (ID $\geqslant$ 525) from Hsu et al. (2012), and their accretion properties, i.e., CTTS or WTTS. For the CTTSs, we estimated their accretion rates from the $\mathrm{H} \alpha$ and $\mathrm{H} \beta$ line luminosities using the methods described in Section 3.4.2. The results are also listed in Table 4. The typical uncertainties are a factor of $\sim 5$ for the accretion rates derived from $\mathrm{H} \alpha$ line luminosity, and a factor of $\sim 3$ for those from $\mathrm{H} \beta$. For the WTTSs with disks, only upper limits on the accretion rate, estimated with $\mathrm{H} \alpha$ and $\mathrm{H} \beta$ line luminosities as done for CTTSs, are given since their Balmer emission is 
Table 6

Accretion Properties of the YSOs in L1641 from Hectochelle

\begin{tabular}{|c|c|c|c|c|c|c|c|c|c|}
\hline ID & $\begin{array}{l}\text { R.A. } \\
\text { (J2000) }\end{array}$ & $\begin{array}{l}\text { Decl. } \\
\text { (J2000) }\end{array}$ & $\begin{array}{c}\mathrm{EW}(\mathrm{Li}) \\
(\AA)\end{array}$ & $\begin{array}{c}\mathrm{EW}(\mathrm{H} \alpha) \\
(\AA)\end{array}$ & $\begin{array}{c}\mathrm{FW}_{\mathrm{H} \alpha, 10 \%} \\
\left(\mathrm{~km} \mathrm{~s}^{-1}\right)\end{array}$ & TTS & $\begin{array}{l}\text { Accretor } \\
\text { Prob }\end{array}$ & $\begin{array}{l}\log \dot{M}_{\mathrm{acc}} \\
\left(M_{\odot} / \mathrm{yr}\right)\end{array}$ & Epoch \\
\hline \multirow[t]{2}{*}{1} & 053605.00 & -064243.8 & .. & -25.2 & 357 & $\mathrm{C}$ & $100 \%$ & -9.4 & 2010 Feb 5 \\
\hline & & & 0.30 & -21.1 & 348 & $\mathrm{C}$ & $100 \%$ & -9.5 & 2010 Mar 3 \\
\hline 2 & 053510.69 & -063415.5 & $\ldots$ & -41.1 & 215 & $\mathrm{C}$ & $83 \%$ & -10.8 & 2010 Nov 29 \\
\hline \multirow[t]{2}{*}{3} & 053452.29 & -063508.5 & $\ldots$ & -10.8 & 292 & W & $96 \%$ & $\ldots$ & 2010 Feb 5 \\
\hline & & & $\begin{array}{l}\cdots \\
\ldots\end{array}$ & -8.3 & 229 & W & $86 \%$ & $\ldots$ & 2010 Mar 3 \\
\hline 4 & 053550.56 & -063317.3 & $\ldots$ & -4.9 & 104 & W & $10 \%$ & $\ldots$ & 2010 Nov 29 \\
\hline \multirow[t]{2}{*}{6} & 053526.58 & -061909.0 & $\cdots$ & -4.3 & 192 & W & $77 \%$ & $\cdots$ & 2010 Nov 29 \\
\hline & & & $\cdots$ & -5.3 & 214 & W & $83 \%$ & $\cdots$ & 2010 Mar 3 \\
\hline
\end{tabular}

(This table is available in its entirety in a machine-readable form in the online journal. A portion is shown here for guidance regarding its form and content.)
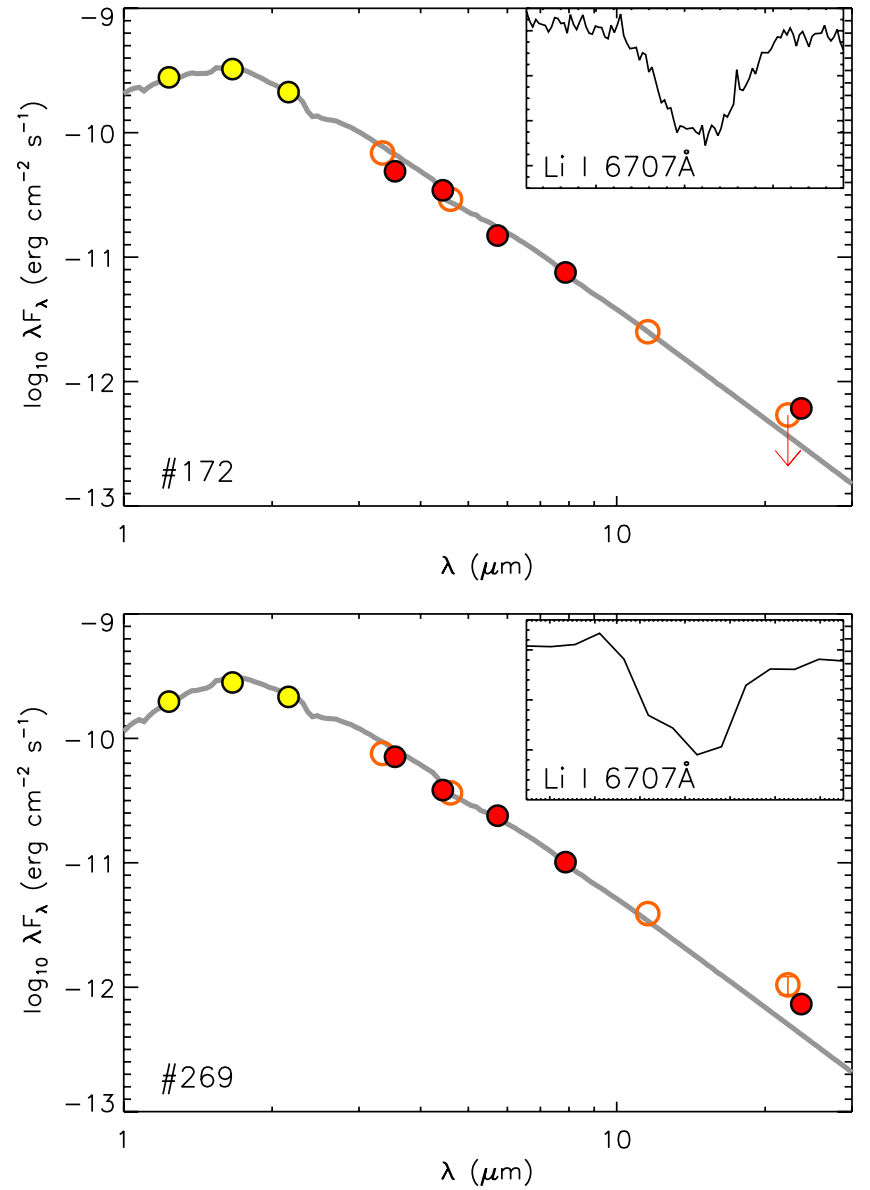

Figure 17. SEDs of debris disk candidates in L1641. The filled circles show the photometry from 2MASS and Spitzer. The open circles are the photometry from WISE. The photospheric emission level is indicated with a gray curve in each panel. The inset in each panel shows the Li I $\lambda 6708$ absorption line. The $\mathrm{Li}$ absorption line is from the Hectochelle spectra for star 172, and from the Hectospec spectra for star 269.

(A color version of this figure is available in the online journal.)

mainly due to the chromospheric activities. In Table 4, the disk property of each YSO is also listed. For the normal full disks, the disks categorized into optically thick disks if $\alpha_{3.6-8} \geqslant-1.8$, or optically thin disks when $\alpha_{3.6-8}<-1.8$.

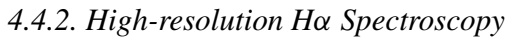

We obtained the $\mathrm{H} \alpha$ emission lines for 235 young stars in L1641 with Hectochelle. In this sample, 8 objects are Class I/

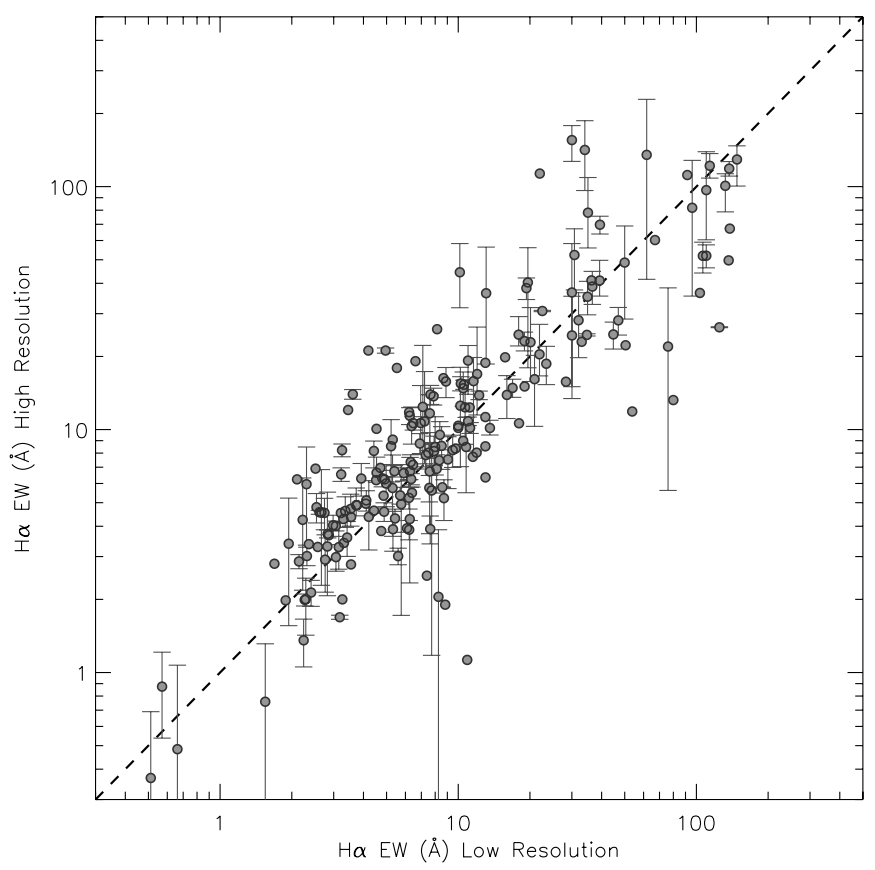

Figure 18. Comparison of the $\mathrm{H} \alpha \mathrm{EW}$ measured from medium-resolution (VIMOS and Hectospec, in Paper I and this work) and high-resolution (Hectochelle) spectra. Note that the high- and low-resolution spectra are taken at different times. The dashed line marks where the two EWs are equal. For the sources that have been observed at more than one epoch, their average EW is used for the plot and the error bar represents their minimum and maximum EWs.

flat sources, 102 are Class II sources, and 124 are Class III sources. One object (385) in the sample cannot be classified due to the lack of photometric data in infrared bands. During 2010-2011, a major fraction of these YSOs have been observed at two or three epochs. In Table 6, we list the EW and $\mathrm{FW}_{\mathrm{H} \alpha, 10 \%}$ of the $\mathrm{H} \alpha$ emission line, the $\mathrm{EW}$ of $\mathrm{Li}$ I $\lambda 6708$ absorption line, types (CTTS or WTTS) of T Tauri stars, and the accretor probabilities. The accretor probabilities are estimated from $\mathrm{FW}_{\mathrm{H} \alpha, 10 \%}$ using the method described in Appendix A. The accretion rates estimated from the $\mathrm{FW}_{\mathrm{H} \alpha, 10 \%}$ are also listed in Table 6.

Many of the YSOs in our Hectochelle sample have been observed in our medium-resolution spectroscopic survey with VIMOS and Hectospec (in Paper I and this work). In Figure 18, we compare the $\mathrm{H} \alpha$ EWs from Hectochelle and those from VIMOS or Hectospec. We find that there is a better agreement between these observations for $\mathrm{H} \alpha \mathrm{EW} \lesssim 10 \AA$ than 

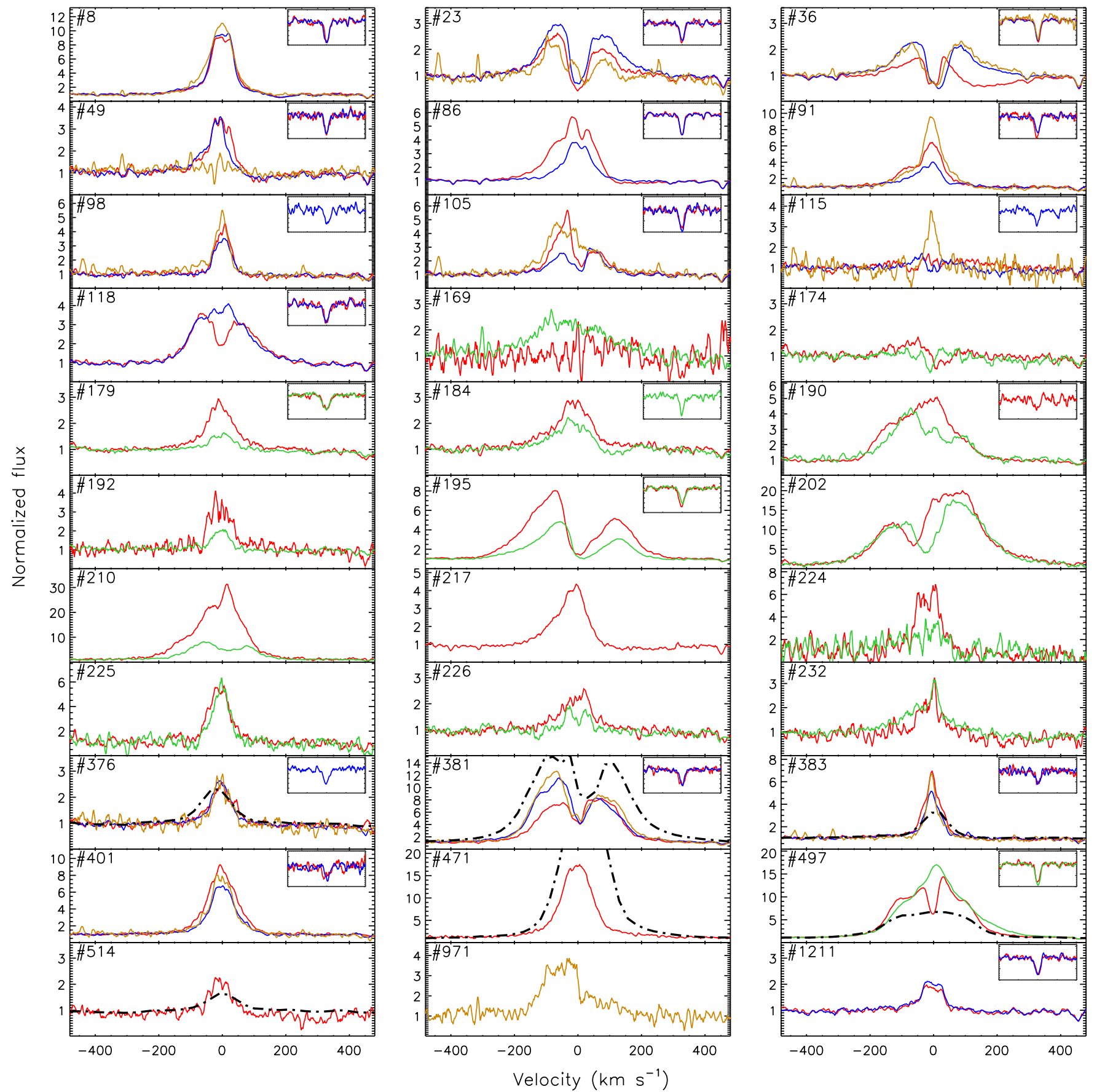

Figure 19. H $\alpha$ profiles at several epochs for TDs in L1641. The observation dates have been encoded as different colors: red for 2010 February 5 , blue for 2010 March 3 , yellow for 2010 November 29, and green for 2011 October 19. The dash-dotted lines display the $\mathrm{H} \alpha$ profiles $(R \sim 2500)$ from Paper I, which was observed with VIMOS. The insets show the Li I $\lambda 6708$ absorption line at several epochs. In each panel, the spectra have been binned up by a factor of five for clarity.

(A color version of this figure is available in the online journal.)

for $\mathrm{H} \alpha \mathrm{EW} \gtrsim 10 \AA$. This can be attributed to the accretion variability of accreting YSOs which usually show $\mathrm{H} \alpha$ $\mathrm{EW}>10 \AA$.

4.4.2.1. H $\alpha$ emission profiles.In Figures 19-23, we display the Hectochelle $\mathrm{H} \alpha$ line profiles for the disk population in our spectroscopic sample. For the sources which have been observed at multiple epochs, all the data are shown as a comparison. In general, most of these line profiles can be classified into the four groups according to the schemes in Reipurth et al. (1996): (I) profiles with a single peak and either no or very weak absorption features, (II) profiles with two relatively equal peaks, (III) profiles with one primary strong peak and one weak secondary peak, and (IV) P Cygni or inverse P Cygni profiles. In our YSO sample, the Type I H $\alpha$ line profiles are either narrow or broad, while the Type II and III profiles are typically broad. We only have one source (136; see the detail description of this source in Section 4.5.3) showing a P Cygni profile (Type IV). One source (69) showed a triple-peak profile on 2010 March 3 and cannot be classified into any of the four groups in Reipurth et al. (1996). 

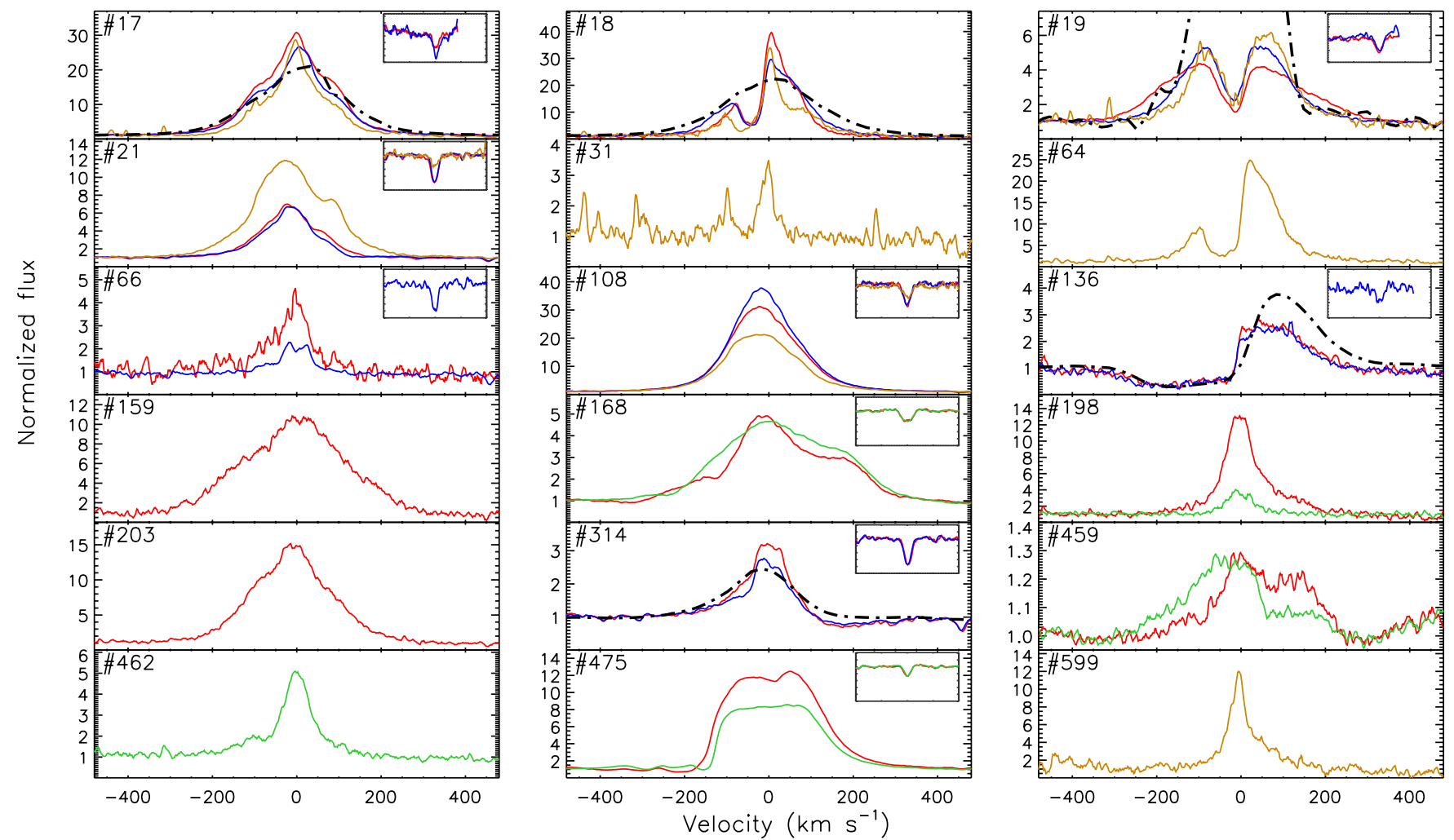

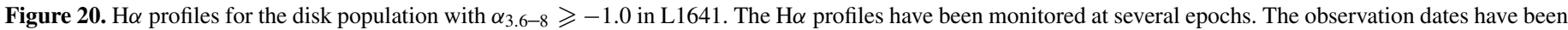

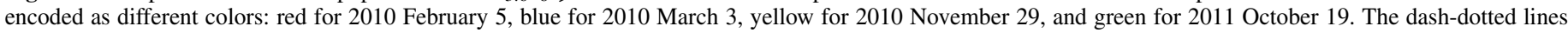

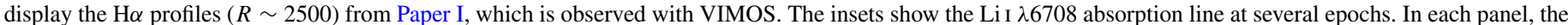
spectra have been binned by a factor of five for clarity.

(A color version of this figure is available in the online journal.)

In our spectroscopic sample observed with Hectochelle, there are 33 TDs. Their $\mathrm{H} \alpha$ line profiles are shown in Figure 19. Among the 33 TDs, $\sim 42 \%$ show broad $\mathrm{H} \alpha$ line profiles $\left(\mathrm{FW}_{\mathrm{H} \alpha, 10 \%}>250 \mathrm{~km} \mathrm{~s}^{-1}\right)$ characteristic of ongoing accretion. Among the TDs showing broad $\mathrm{H} \alpha$ line profiles, five sources (23, 36, 195, 202, and 381) display $\mathrm{H} \alpha$ line profiles with double peaks from the data observed at all epochs. As defined in this work, TDs show very weak or no excess emission in shorter IRAC bands. The absence of excess emission at such wavelengths can be explained if (1) TDs have dissipated most or all of the hot inner disks or (2) in the inner regions of TDs there are opacity holes due to a lack of small dust grains while gas still exists in these regions. For the accreting TDs in our sample, scenario (1) can be excluded.

In Figure 20, we show the line profiles for the disk population with $\alpha_{3.6-8} \geqslant-1.0$. These sources have strong excess emission in the IRAC bands, and thus are expected to show strong and broad $\mathrm{H} \alpha$ line profiles. In the figure, $\sim 83 \%$ of the sources display broad line profiles $\left(\mathrm{FW}_{\mathrm{H} \alpha, 10 \%}>250 \mathrm{~km} \mathrm{~s}^{-1}\right)$ at one epoch. Five sources $(31,66,314,462$, and 599) exhibit relatively narrow line profiles and these sources may have more quiescence accretion activities.

In Figures 21 and 22, we show the line profiles for the sources with $-1.0>\alpha_{3.6-8} \geqslant-1.8$. These sources are typical Class II sources with optically thick inner disks. In this sample, $\sim 80 \%$ of the sources show broad $\mathrm{H} \alpha$ line profiles $\left(\mathrm{FW}_{\mathrm{H} \alpha, 10 \%}>250 \mathrm{~km} \mathrm{~s}^{-1}\right)$ at one epoch. In Figure 23, we display the $\mathrm{H} \alpha$ line profiles of the YSOs with $-1.8>\alpha_{3.6-8} \geqslant-2.5$. The YSOs with such $\alpha$ values are considered as having optically thin inner disks. However, four sources (16, 54, 138, and 162) still show very broad $\mathrm{H} \alpha$ line profiles $\left(\mathrm{FW}_{\mathrm{H} \alpha, 10 \%}>250 \mathrm{~km} \mathrm{~s}^{-1}\right)$, suggesting that they are still accreting.

4.4.2.2. $\mathrm{H} \alpha$ line variability. $\mathrm{H} \alpha$ line profile fluctuations are very common in CTTSs, and can be due to the variability of accretion flow or outflow (Johns \& Basri 1995; Gullbring et al. 1996; Alencar et al. 2001; Alencar \& Batalha 2002). In our sample, $\sim 90$ diskless YSOs and $\sim 80$ disked YSOs have had their $\mathrm{H} \alpha$ line profiles monitored at multiple epochs with Hectochelle. The $\mathrm{H} \alpha$ emission-line variations of WTTSs are mainly related to the chromospheric activity, and are less variable than the CTTSs. In this work, we will only focus on the CTTSs. In our sample, most of CTTSs show clear variations in their $\mathrm{H} \alpha$ line profiles. Among them, several sources, e.g., sources 20, 26, 69, exhibit extreme line variations. The $\mathrm{H} \alpha$ line fluctuations (see Figures 19-23) can be mainly grouped into several types: (1) $\mathrm{H} \alpha$ line profiles vary from single peak to double peak, or even to triple peaks, e.g., sources 26,69 , and 339 , (2) the peak positions vary, e.g., sources 93,110 , and 195 , (3) the ratio between two peaks of $\mathrm{H} \alpha$ lines varies, e.g., the sources 343 and 381, and (4) $\mathrm{H} \alpha$ line profiles broaden or narrow, e.g., sources 19,21 , and 86 . The $\mathrm{H} \alpha$ line variation of one YSO can usually be classified into more than one group. For the YSOs which can be categorized into group (4), their $\mathrm{H} \alpha$ line variations are mainly due to their accretion variations. Specifically, two sources in our sample (26 and 86) show large variability from CTTS $\left(\mathrm{FW}_{\mathrm{H} \alpha, 10 \%}>250 \mathrm{~km} \mathrm{~s}^{-1}\right)$ to WTTS $\left(\mathrm{FW}_{\mathrm{H} \alpha, 10 \%} \leqslant 250 \mathrm{~km} \mathrm{~s}^{-1}\right)$.

A few of the sources in our Hectochelle sample have been observed with VIMOS in 2008 January or March. These data have been presented in Paper I. As a comparison, we show 

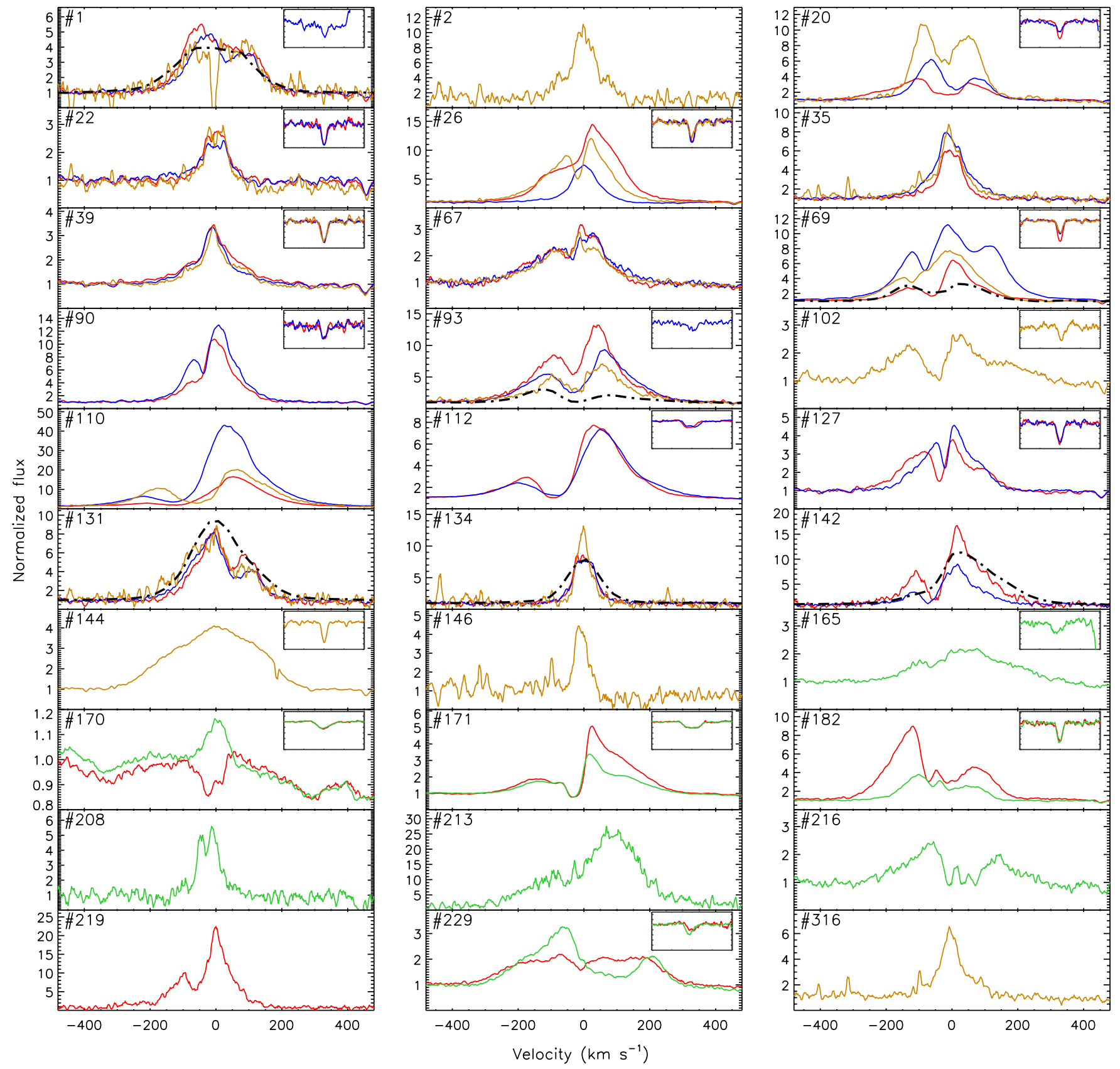

Figure 21. Similar to Figure 20 but for the disk population with $-1.0>\alpha_{3.6-8} \geqslant-1.8$ in L1641.

(A color version of this figure is available in the online journal.)

these observations in Figures 19-23. The spectral resolution of the VIMOS spectra is $\sim 2500$ and the data can only resolve relatively broad $\mathrm{H} \alpha$ line profiles. The comparison shows that sources 69, 344, and 470 exhibit large variations in $\mathrm{H} \alpha$ emission line.

\subsection{Exotic Objects}

\subsubsection{Extremely Embedded Sources}

In the [3.6] - [4.5] versus [5.8] - [8.0] color-color diagram (see Figure 6(a)), five objects (sources 1074, 1218, 1220, 1233, and 1244) have colors distinct from the other sources. Their colors $([5.8]-[8.0] \sim 0$ and [3.6] $-[4.5]>1.0)$ make them appear as diskless stars with extremely high extinction $\left(A_{\mathrm{K}} \gtrsim 10\right)$. Among the five sources, four (1074, 1220, 1233, and 1244) are in the FOVs of the XMM surveys, and only source 1074 is detected. In the Spitzer IRAC images, four sources (IDs 1218, 1220, 1233, and 1244) are surrounded by extended emission with fan-shaped structures (see Figure 24), and only source 1074 appears as a point source without any nebulosity. Source 1074 is not detected in the MIPS $24 \mu \mathrm{m}$ images. Thus, we consider it to be a highly reddened diskless star. The four sources associated with fan-shaped nebulae are all detected at $24 \mu \mathrm{m}$, and show strong excess emission in this band with [8.0] $-[24] \gtrsim 7$, indicating they could be at a very early stage in the star formation process. The fan-shaped nebulae around these sources are most easily explained by the scattering of photons from the central star out of cone-shaped cavities which are carved out of the dense envelope by energetic outflows. Furthermore, near source 1244 we find bipolar jets traced by the 

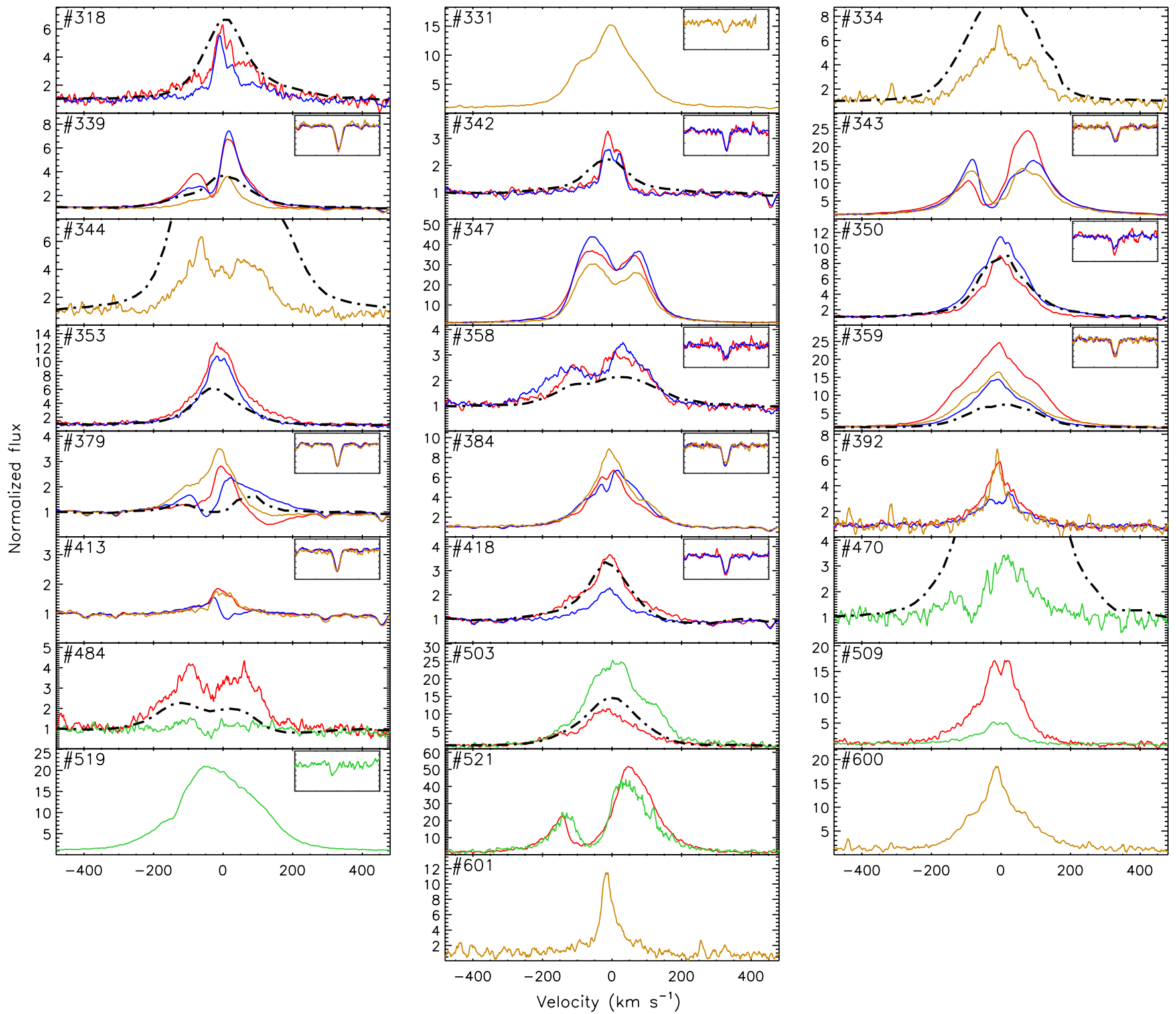

Figure 22. Similar to Figure 21, but for different targets.

(A color version of this figure is available in the online journal.)

Spitzer $4.5 \mu \mathrm{m}$ emission. ${ }^{8}$ The morphology of the jets suggests that source 1244 could be its driving source.

We have searched for the five sources in the Hubble Space Telescope (HST) data archive. Two objects (1233 and 1244) have been observed with $H S T$ /WFC3 at the $F 160 \mathrm{~W}$ band, and are not detected in these images. Source 1220 has been observed with $H S T /$ NIC2 in the $F 160 W$ and $F 205 W$ bands. In addition to continuum emission, the broad bands $F 160 \mathrm{~W}$ and $F 205 \mathrm{~W}$ principally transmit the [Fe II] $1.644 \mu \mathrm{m}$ and $\mathrm{H}_{2} 2.122 \mu \mathrm{m}$ lines, respectively, both of which can originate from shocked gas. In the HST images at both the $F 160 \mathrm{~W}$ and $F 205 \mathrm{~W}$ bands, object 1220 shows a cometary shape with a bright head and a long tail. Figure 25 shows the $F 205 W$ image of object 1220 . Two scenarios can explain the shape of object 1220 in the HST images: (1) a young stellar system photoevaporated by nearby

8 The Spitzer $4.5 \mu \mathrm{m}$ band covers several $\mathrm{H}_{2}$ lines $(\mu=0-0, S(9,10,11))$, which could be excited by the interaction of the outflows with the ambient medium. Thus, the Spitzer $4.5 \mu \mathrm{m}$ images are used to search for the jets from young stars (see, e.g., Zhang \& Wang 2009). massive stars, e.g., like proplyds (O'dell et al. 1993; Brandner et al. 2000; Fang et al. 2012), or (2) a YSO producing a jet which appears as a long tail. For object 1220, the former scenario can be excluded since there are no massive stars near object 1220 . The latter scenario could be a promising explanation for the shape of object 1220 in the HST images.

\subsubsection{A New Subluminous Object}

In the H-R diagram (see Figure 10), one source (143) appears to be subluminous by a factor of $\sim 110$ with respect to a $1 \mathrm{Myr}$ PMS star of a similar spectral type. Its optical spectrum and SED are shown in Figure 26. In its optical spectrum, the $\mathrm{H} \alpha$ emission line seems to be quite strong. Objects with similar properties have been found in many regions, e.g., L1630N, the Lupus 3 dark cloud, and Taurus (Paper I; Comerón et al. 2003; White \& Hillenbrand 2004). Typically, these exotic objects show abnormally large Balmer line EWs, and normal EWs of He I $5876 \AA$, and the Ca II near-infrared triplet (8498, 8542, and 8662 A; see Paper I; Comerón et al. 2003). One likely scenario 


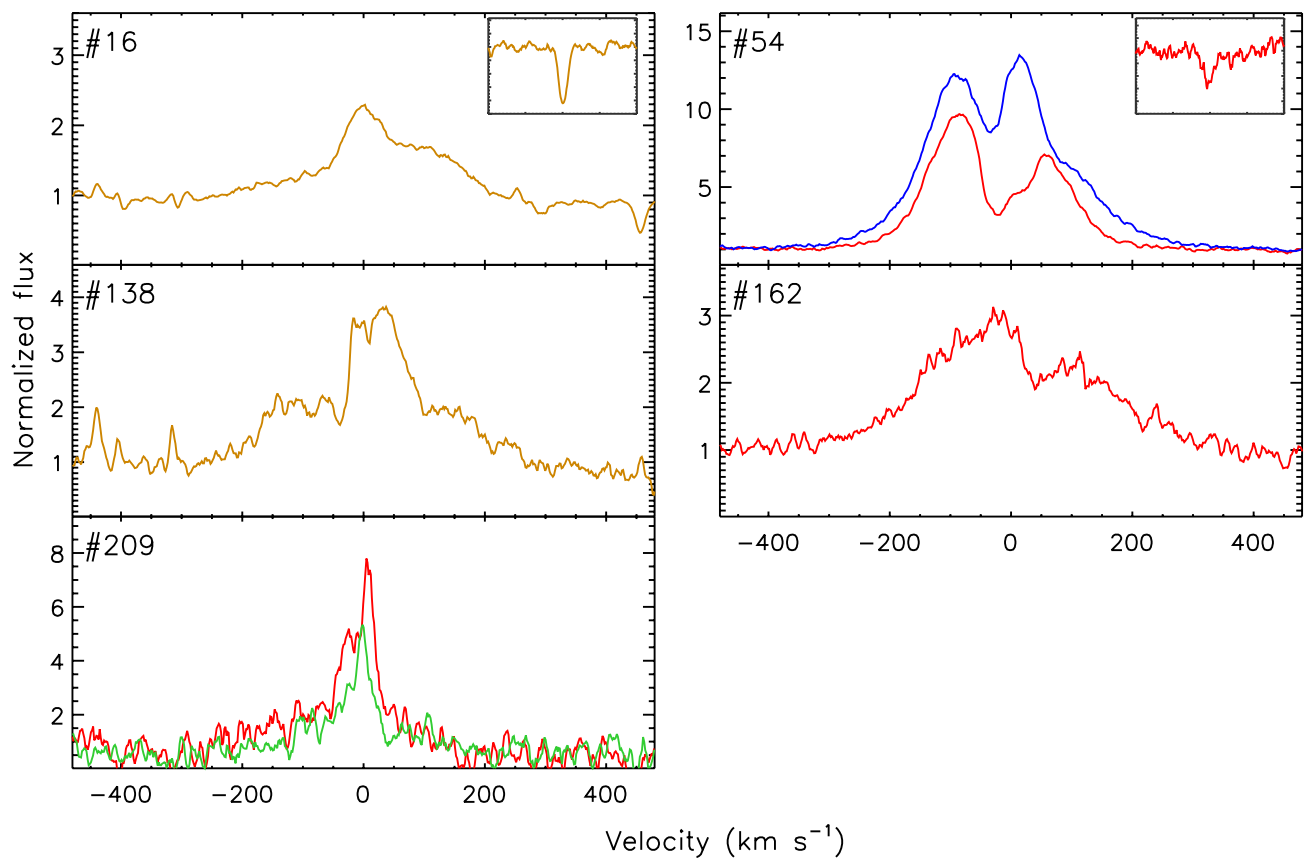

Figure 23. Similar to Figure 21 but for the disk population with $\alpha_{3.6-8}<-1.8$ in L1641.

(A color version of this figure is available in the online journal.)
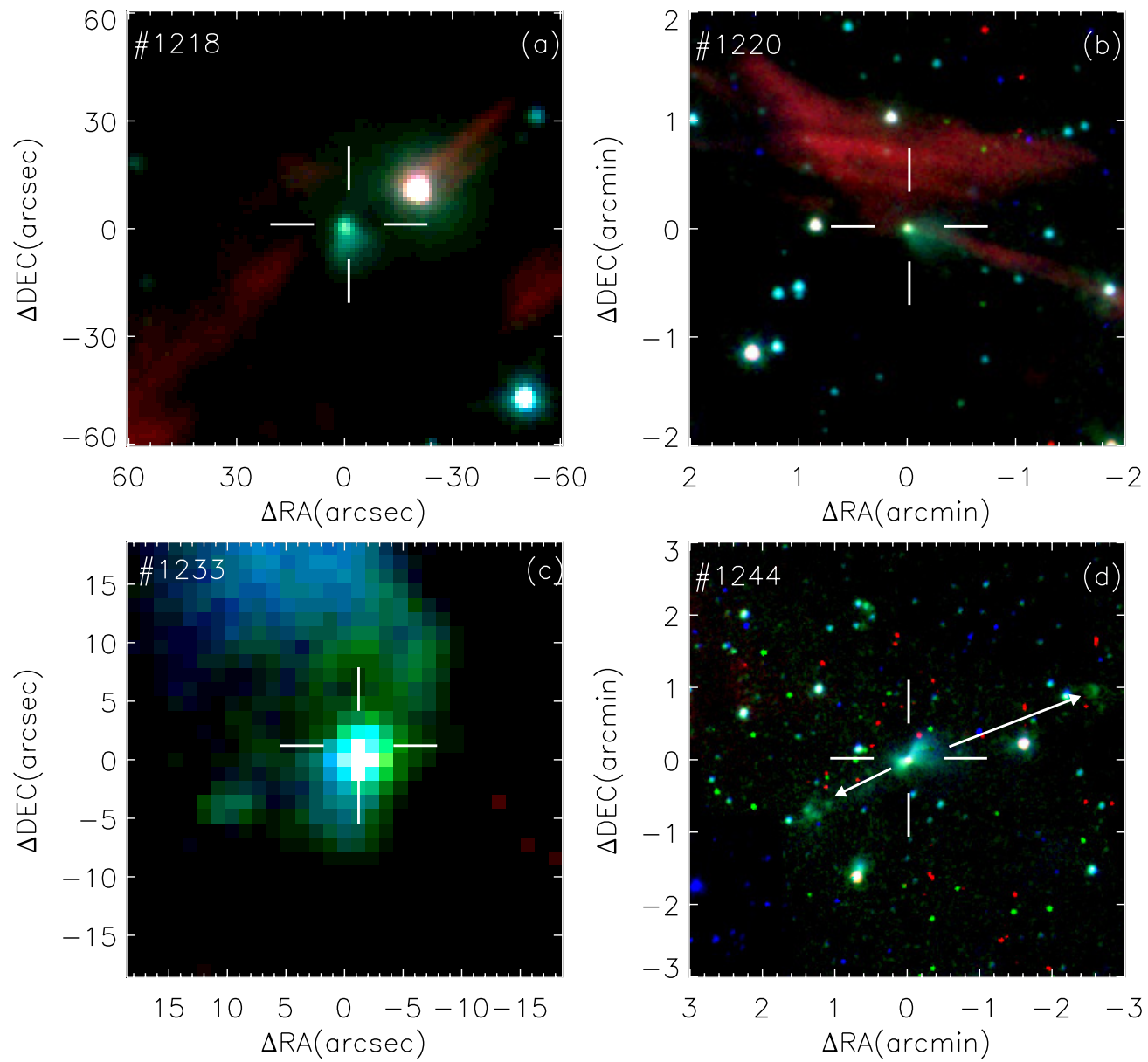

Figure 24. Spitzer color images (red: $8 \mu \mathrm{m}$; green: $4.5 \mu \mathrm{m}$; and blue: $3.6 \mu \mathrm{m}$ ) centered on the four source 1218 (a), 1220 (b), 1233 (c), and 1244 (d) with [3.6] $-[4.5] \sim 0$ and [5.8] $-[8.0]>1$. The arrows in panel (d) show the directions of a bipolar jet.

(A color version of this figure is available in the online journal.) 


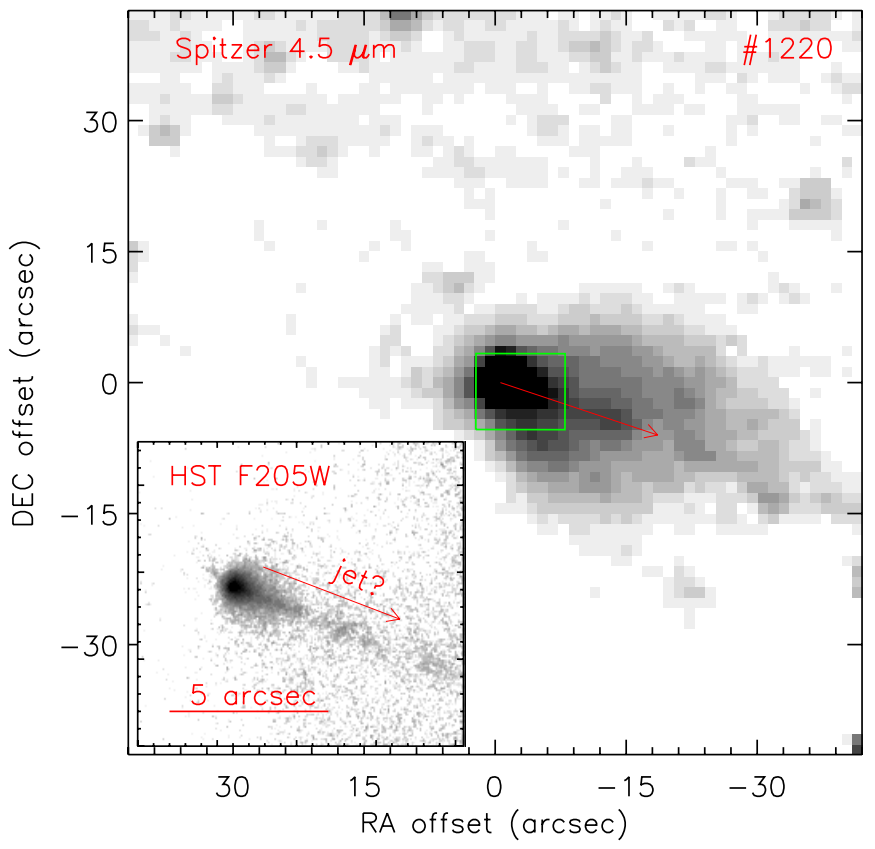

Figure 25. Spitzer $4.5 \mu \mathrm{m}$ image centered on source 1220. The inset shows the HST F $205 \mathrm{~W}$ image of source 1220 with the sample FOV shown with the box on the $4.5 \mu \mathrm{m}$ image. The arrows show the direction of a probable jet from source 1220 detected in the HST image.

(A color version of this figure is available in the online journal.)

for these exotic objects is that they are harboring flared disks with high inclinations. In this case, the stellar photospheric light is largely absorbed by the material in the cold, flared outer disk. The light that we can see mainly comes from the photon scattering off the disk surface, and is therefore much reduced. The optical emission lines with large EWs, e.g., Balmer lines and forbidden oxygen emission lines, may arise in an outflow or disk wind whose scale is much larger than the central star allowing at least part of the line flux to reach us relatively unscreened. Emission lines like He I $5876 \AA$, and the Ca II nearinfrared triplet $(8498,8542$, and $8662 \AA$ ) that mainly form in the magnetospheric infall flows, close to the central stars, should be as obscured as the photospheric continuum.

We employ the SED fitting tool of Robitaille et al. (2007) to fit the SED of source 143. We excluded models with stellar spectral types more than two subclasses different from the observation, and defined the good-fit models as the models with $\chi^{2}-\chi_{\text {best }}^{2}<2 n_{\text {data }}$, where $\chi_{\text {best }}^{2}$ is the $\chi^{2}$ of the bestfitting model and $n_{\text {data }}$ is the number of data points for the fits. We obtained two good-fit models (Model IDs 3002401 and 3002775) which are shown in Figure 26(b). The disk inclination angles of both models are $\sim 87^{\circ}$ which supports the hypothesis that the subluminous objects are harboring disk systems with high inclinations. The SED fits also give a better constraint on the stellar luminosity of source 143. From the stellar luminosity, in combination with the stellar effective temperature from the optical spectrum, its mass and age are estimated to be 2.0-2.2 $M_{\odot}$ and 0.4-0.6 Myr from D08 tracks, 2.6-2.7 $M_{\odot}$ and 1.3-1.8 Myr from S00, 1.8-2.0 $M_{\odot}$ and 0.4-0.6 Myr from DM97, and 1.9 $M_{\odot}$ and 1.7-2.4 Myr from Pisa11. These age estimates are comparable to the ages of other YSOs in L1641 from each PMS evolutionary model.

Though the two good-fit models generally follow the shape of the observed SED for source 143, they fail to fit the $10 \mu \mathrm{m}$ silicate feature (see Figure 26(b)). The observed $10 \mu \mathrm{m}$ silicate feature of source 143 is seen in emission, suggesting that its disk inclination should be substantially different from $90^{\circ}$ (edge-on) since the edge-on disk system usually shows the $10 \mu \mathrm{m}$ silicate feature in absorption (Pontoppidan et al. 2005). More detailed models as well as observations are required to better constrain the nature of object source 143 .

\subsubsection{The FU-Ori-Type Object Candidate}

Source 136 is one of the most interesting YSOs in our sample. It shows a $\mathrm{P}$ Cygni profile in the $\mathrm{H} \alpha$ line in all of our spectra observed with VIMOS, Hectochelle, and Hectospec (see Figure 20). In Paper I, we proposed this object as an FU Ori candidate. This object has been further investigated in Caratti O Garatti et al. (2012). It shows a $10 \mu \mathrm{m}$ silicate feature in absorption (Caratti O Garatti et al. 2012), suggesting it is probably a Class I protostar. This source also shows a blueshifted He I line in absorption (Caratti O Garatti et al. 2012), a characteristic feature of FU Ori objects (Connelley \& Greene 2010). However, the non-detection of absorption features of the $\mathrm{CO}$ band-head lines longward of $2.29 \mu \mathrm{m}$ make this source less likely to be of the FU-Ori-type (Caratti O Garatti et al. 2012). We have collected the near-infrared photometric data of source 136 in the literature (Strom et al. 1989a; Chen \& Tokunaga 1994;
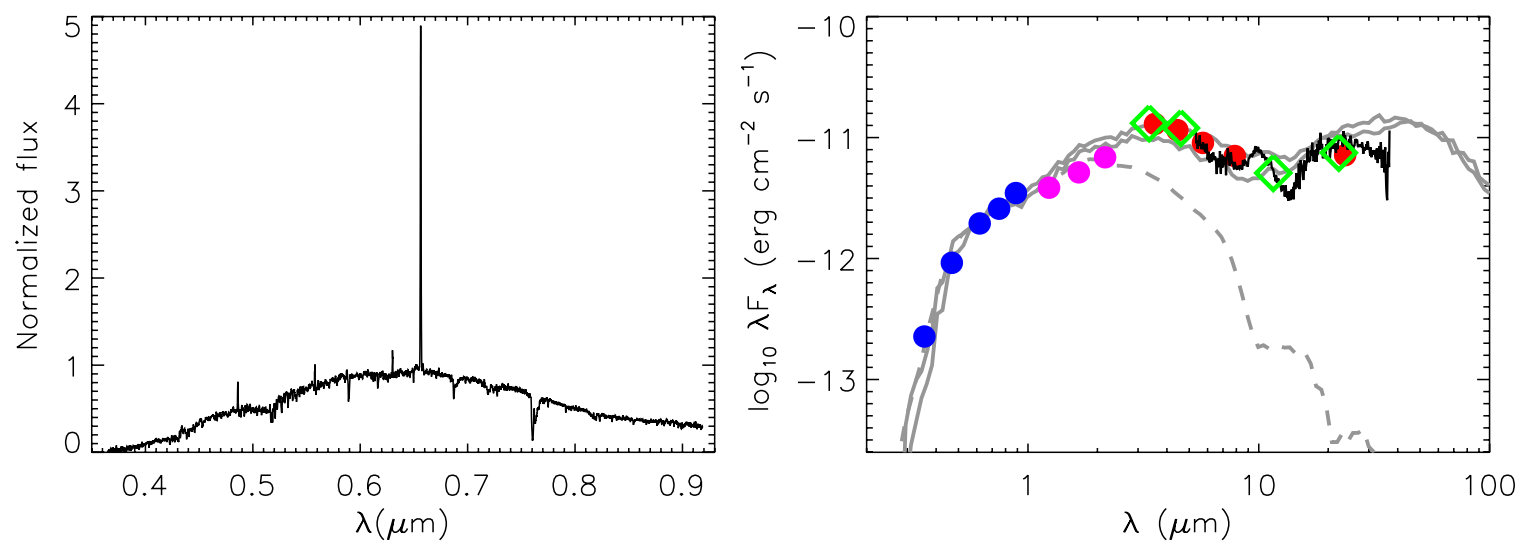

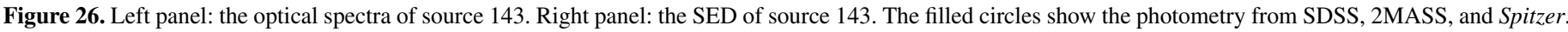

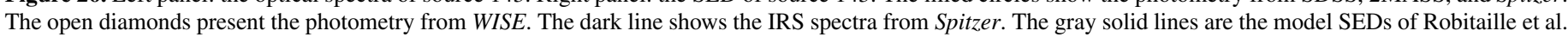
(2007) which best fit to the observed SED of source 143. The gray dashed line shows the photospheric level.

(A color version of this figure is available in the online journal.) 
Skrutskie et al. 2006). The data span epochs from 1985/1986 to 2009 during which the $K$ magnitudes of the source range from 8.14 to 8.39 . Thus, it seems that source 136 did not show big change on its brightness, which furthermore argues against it as an FU Ori type object.

\section{DISCUSSION}

\subsection{Disk Evolution \\ 5.1.1. Disk Frequency}

In the YSO catalog of L1641, 1314 sources can be grouped into one of the four classes, i.e., Class I, flat-spectrum, Class II, and Class III. The TDs in our sample are considered to be Class II sources. We calculate the disk fraction as $N(\mathrm{II}) / N(\mathrm{II}+\mathrm{III})$, where $N($ II) and $N($ III) are the number of Class II and Class III sources, respectively, as done for Taurus by Luhman et al. (2010). The disk fraction of our YSO sample is estimated to be $\sim 51 \% \pm 2 \%(533 / 1040)$. If we count only the YSOs with $\mathrm{X}$-ray emission, then the disk fraction is $\sim 36 \% \pm 3 \%(166 / 466)$. The lower disk fraction among the X-ray-emitting YSOs, compared with the whole YSO sample, may be due to the $X M M$ observations detecting more Class III sources than Class II sources since X-ray observation is more sensitive to the former than the latter (Feigelson \& Montmerle 1999). As shown in Figure 2, there is a slight difference on the FOVs of the XMM survey and Spitzer survey. If we only count the YSOs within the common observed regions of both surveys, then the disk fraction is $\sim 51 \% \pm 2 \%(448 / 884)$ for all the cataloged YSOs and the disk fraction is $\sim 49 \% \pm 3 \%(278 / 567)$ for "bright" YSOs $\left(K_{\mathrm{s}} \leqslant 12.5 \mathrm{mag}\right)$. As discussed in Section 4.1.2, the census of diskless population stars with $X M M$ data is relatively complete for Class III sources with $K_{\mathrm{s}} \leqslant 12.5 \mathrm{mag}$, and becomes very incomplete for fainter sources. Thus, we may expect a much higher disk fraction for all the cataloged YSOs than for the brighter YSOs $\left(K_{\mathrm{s}}>12.5 \mathrm{mag}\right)$. However, we do not see a significant difference in the disk fractions for the two populations. In this work, $\sim 460$ Class III sources are identified from several spectroscopic surveys (Allen 1995; Hsu et al. 2012; Paper I; this work). These surveys have selected YSO candidates for observations partially from the optical color-magnitude diagrams. In these surveys, more than 200 Class III sources without X-ray emissions have been identified, which alleviates the incompleteness of Class III sources that our YSO census suffered from the $X M M$ survey. In general, we conclude that the disk fraction in L1641 is $\sim 50 \%$.

The disk fraction in L1641 is slightly lower than that in Taurus $(59 \% \pm 4 \%)$ which is at an age similar to that of L1641 (Luhman et al. 2010). In Taurus, most YSOs have been observed with mid-IR spectroscopy, which can be used to distinguish the YSOs with envelopes from those without envelopes (Furlan et al. 2006). Using these data, 59\% of the "flat-spectrum" YSOs are classified into Class II (Luhman et al. 2010). Assuming that the same fraction of the "flat-spectrum" YSOs in L1641 is actually Class II sources, the disk fraction in L1641 would increase to $54 \% \pm 2 \%$, which is generally consistent with that in Taurus $(59 \% \pm 4 \%)$.

Furthermore, in L641, we have found 73 TDs. Among them, 65 have been confirmed and 8 are TD candidates. The TDs count for $14 \%$ of the disk population (Class II) in our YSO sample. Under the assumption that the Class II lifetime is $2 \mathrm{Myr}$, the lifetime of TDs is estimated to be $\sim 0.3 \mathrm{Myr}$.

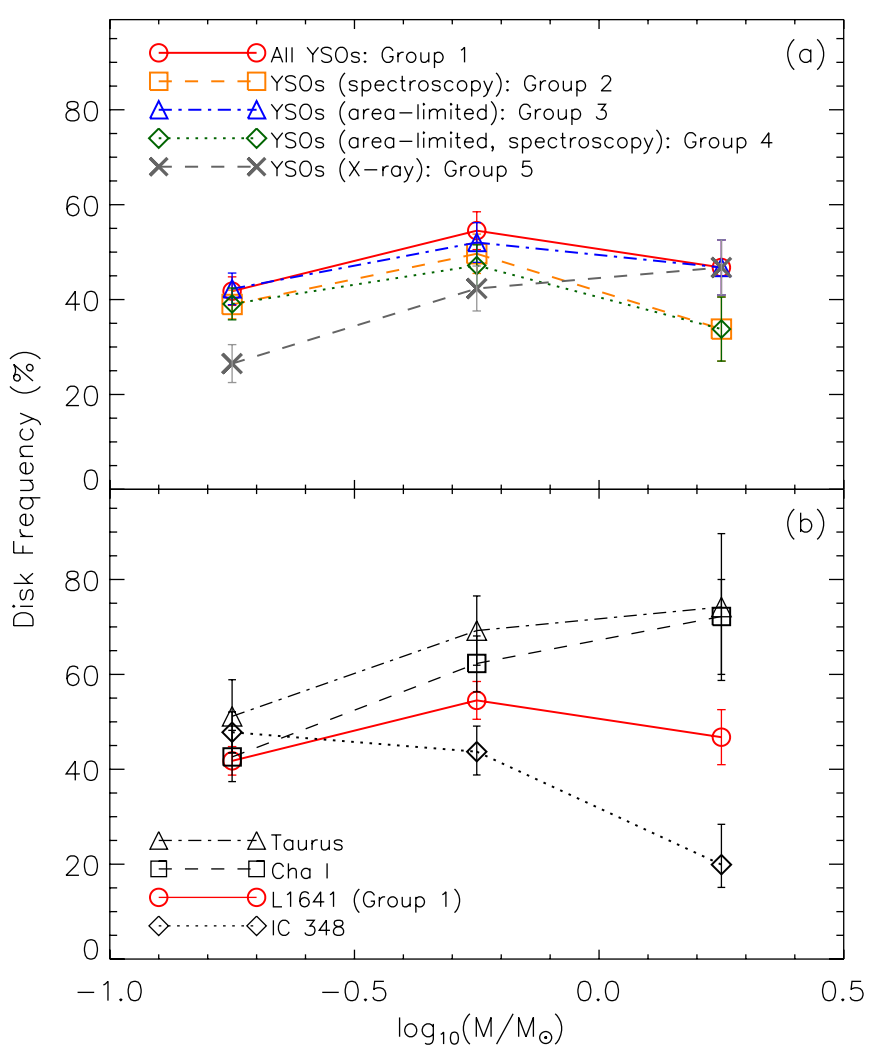

Figure 27. (a) Disk frequency as a function of stellar mass for all YSOs with masses $\geqslant 0.1 M_{\odot}$ in L1641 (open circles), for all YSOs with spectroscopy (open squares), YSOs within the common regions covered by the XMM and Spitzer surveys (area-limited, open triangles), area-limited YSOs with spectroscopy (open diamonds), and YSOs with X-ray emission (crosses). (b) The disk frequency as a function of stellar mass for all YSOs with masses $\geqslant 0.1 M_{\odot}$ in L1641 (open circles), Taurus (open triangles; Luhman et al. 2010), Chamaeleon I (open squares; Luhman et al. 2008), and IC 348 (open diamonds; Lada et al. 2006).

(A color version of this figure is available in the online journal.)

\subsubsection{Disk Frequency as a Function of Stellar Masses}

In our sample of young stars, more than 800 sources have been observed with spectroscopy, thus their mass estimates are relatively reliable. For the other $\sim 200$ YSOs without spectral types, we derived masses from their dereddened $J$-band photometry via the theoretical relation between stellar mass and the $J$-band photometry for PMS stars at an age of 1.5 Myr (the median age of YSOs in L1641) from Dotter et al. (2008). In Figure 27, we show the disk frequency as a function of stellar mass for five groups of YSOs: ${ }^{9}$ (1) all YSOs with estimates of stellar masses from either spectroscopy or $J$-band photometry, (2) all YSOs observed with spectroscopy, (3) all YSOs within the common regions covered by the $X M M$ and Spitzer surveys (see Figure 2), (4) the YSOs among Group 3 with spectroscopy, and (5) the YSOs with X-ray emission. As shown in Figure 27, the trends of disk frequency with stellar mass for the YSOs of groups 1-4 are similar, and their disk frequencies are almost constant as a function of stellar masses with a slight peak at $\log \left(M_{*} / M_{\odot}\right) \approx-0.25$. The disk frequencies of the YSOs with $\mathrm{X}$-ray emission are lower within the sub-solar mass range than those of Group 1-4, and generally increase with increasing stellar masses, which may be because the X-ray observations are very inefficient at detecting the very low mass disked YSOs.

\footnotetext{
9 The mass bins used are, in $\log \left(M_{*} / M_{\odot}\right),-1$ to $-0.5,-0.5$ to 0 , and $>0$.
} 


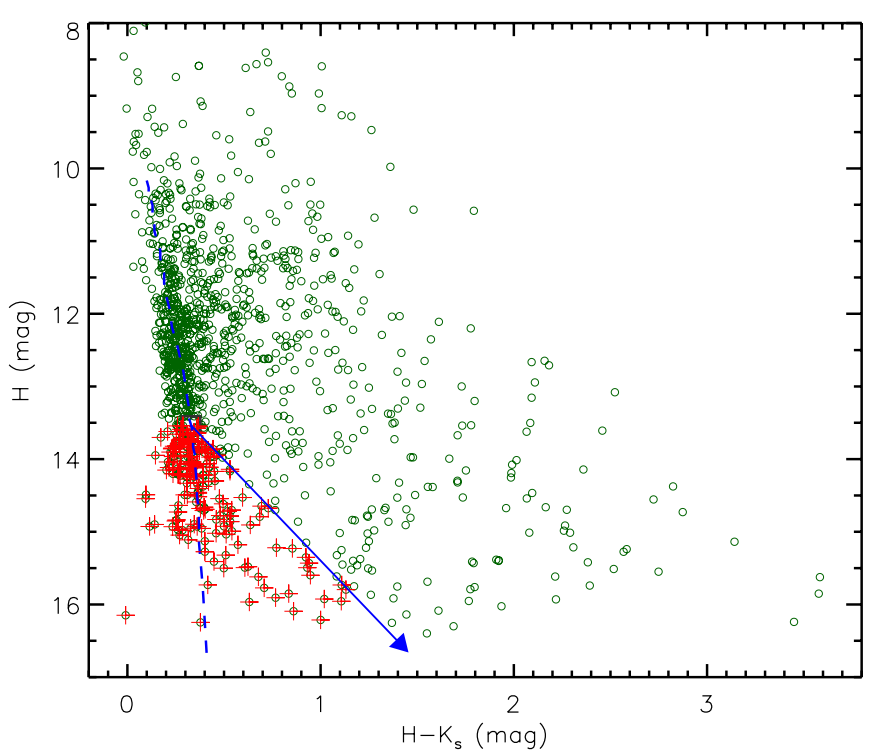

Figure 28. $H$ vs. $H-K_{\mathrm{S}}$ color-magnitude diagram for the YSOs (open circles) in L1641. The dashed line shows the two-megayear PMS isochrone from Baraffe et al. (1998). The open box shows the location of a two-megayear-old low-mass PMS star with a mass of $0.1 M_{\odot}$. The pluses mark the very low mass stellar/ substellar candidates $\left(M_{*} \leqslant 0.1 M_{\odot}\right)$. The arrow shows the reddening vector with a length of $A_{K}=2$ (Rieke \& Lebofsky 1985).

(A color version of this figure is available in the online journal.)

The trend of disk frequency with stellar mass in this work is different from our finding in Paper I, in which the disk frequency increases with increasing stellar mass. In Paper I, we used a limited sample ( 260) of YSOs, only including the spectroscopic YSOs, to construct the disk frequency versus stellar mass relation. In this work, the combination of the XMM and Spitzer imaging surveys make our study less biased. As a comparison, in Figure 27, we also show relations between the disk frequency and stellar mass for YSOs in IC 348, Chamaeleon I, and Taurus (Lada et al. 2006; Luhman et al. 2008, 2010). The trend in L1641 that we identify is quite different from that seen in Taurus (Luhman et al. 2010) or in the two-megayear-old Chamaeleon I (Luhman et al. 2008). In these regions, the disk frequency increases with stellar mass. In the somewhat older IC 348 (see Figure 27), the disk frequency shows a decreasing trend with stellar mass. A similar trend has also been found in the Cep OB3b cluster (3-5 Myr Allen et al. 2012). The trend of disk frequency with stellar mass in L1641 is different from any of the above regions. To interpret the different trends of disk frequency with stellar mass in these regions, one may need to know the disk properties around young stars with different masses, the star formation history (SFH), and the local environment in each region.

\subsubsection{Disks around Very Low Mass Stellar/Substellar Objects}

In our spectroscopic sample, there are three young brown dwarfs (sources 342,398, and 404) according to the evolutionary tracks from Baraffe et al. (1998). Among them, source 404 harbors a circumstellar disk. For the sources without spectral types, we use the $H$ versus $H-K_{\text {s }}$ color-magnitude diagram (Figure 28) to select very low mass stellar/substellar objects by comparing them with the theoretical PMS isochrone. The ages of spectroscopic YSOs in L1641, estimated with the evolutionary tracks from Baraffe et al. (1998), show a broad distribution with a median age of $\sim 2$ Myr. In the figure, we also display the two-megayear PMS isochrone (Baraffe et al. 1998). We select very low mass object candidates that are below the reddening vector of a 2 Myr old, $0.1 M_{\odot}$ PMS star in the $H$ versus $H-K_{\mathrm{s}}$ color-magnitude diagram, and find 161 sources. In this sample, 115 sources have spectral-type estimates in the literature, and $50 \%$ of them have spectral types later than M5, and $83 \%$ of them are later than M4, suggesting a major fraction of our selected candidates are really very low mass objects. Among the 161 very low mass stellar/substellar object candidates, the disk fraction is $47 \%$ (76/161), which is consistent with what we found for all the YSOs in L1641.

Figure 29 shows the SEDs of two brown dwarf candidates (IDs 541 and 941) with disks. The masses of the two sources are estimated to be $\sim 0.04 M_{\odot}$ using the two-megayear PMS isochrone from Baraffe et al. (1998) in Figure 28. The SEDs of the two sources are fitted using the online fitting tool provided by Mayne et al. (2012). The fitting tool employs a set of parameters including stellar mass, accretion rate, and disk mass (see detailed description in Mayne et al. 2012). When fitting the SEDs, we fix the stellar masses to be $0.04 M_{\odot}$, and free other parameters. In Figure 29, we show the 10 best-fitting model SEDs for each source. Among the top 10 best-fitting models, the fitted ages of the two sources are both $1 \mathrm{Myr}$, and the disk radii are around 100-300 AU. From the fitting, the disk around source 541 may have a higher inclination angle $\left(40^{\circ}-60^{\circ}\right)$ and lower mass $\left(4 \times 10^{-4}-4 \times 10^{-5} M_{\odot}\right)$ than that of source 941 , in which the disk is almost face-on and the disk mass is $\sim 4 \times 10^{-4} M_{\odot}$.

\subsubsection{Ages of the Different Populations}

According to traditional low-mass star formation theory, CTTSs evolve into WTTSs as circumstellar disks dissipate. Thus, one would expect that WTTSs are older than CTTSs. However, the observations of many star-forming regions do not support this theory (Herbig 1998; Hartmann 2001; Herbig \& Dahm 2002; Dahm \& Simon 2005), and there are only a few cases in which the WTTSs are found to be older than CTTSs (Hartigan et al. 1995; Bertout et al. 2007). In Paper I, we showed that the WTTSs without disks are statistically older than CTTSs in L1641, though both show age distributions with large spreads. In this work, instead of classifying the YSOs into CTTSs or WTTSs when comparing their age distributions, we divide the YSOs into three groups: YSOs with optically thick disks, TD objects, and diskless YSOs. In this analysis, we include both YSOs from this work and from Paper I.

The age distributions of the three populations are shown in Figure 30. The median ages of the YSOs with optically thick disks, TD objects, and diskless YSOs are $\sim 1.1 \mathrm{Myr}, \sim 1.5 \mathrm{Myr}$, and $\sim 1.8 \mathrm{Myr}$, respectively. Though the median ages of different populations increase with their evolutionary stages, each of them shows a very broad distribution. A Kolmogorov-Smirnov (K-S) test reveals a relatively low probability $\left(P \sim 3 \times 10^{-6}\right)$ for the optically thick disks and diskless YSOs to be drawn randomly from the same age distribution. The probability that the TDs and diskless YSOs are drawn randomly from the same population is also low $(P \sim 0.02)$, whereas the age distributions of the optically thick disks and TDs are less indistinguishable $(P \sim 0.2)$.

Figure 30 shows that $34 \%$ of the diskless YSOs have ages less than 1 Myr. Here, the presence of a disk around a YSO is determined by the excess emission at infrared wavelengths $\lesssim 24 \mu \mathrm{m}$, which is only sensitive to the disk at several AU to tens of AU, depending on the effective temperature of central stars. A few of these "diskless" YSOs may still have disks but with large inner holes. If these YSOs are really diskless or harbor disks with 


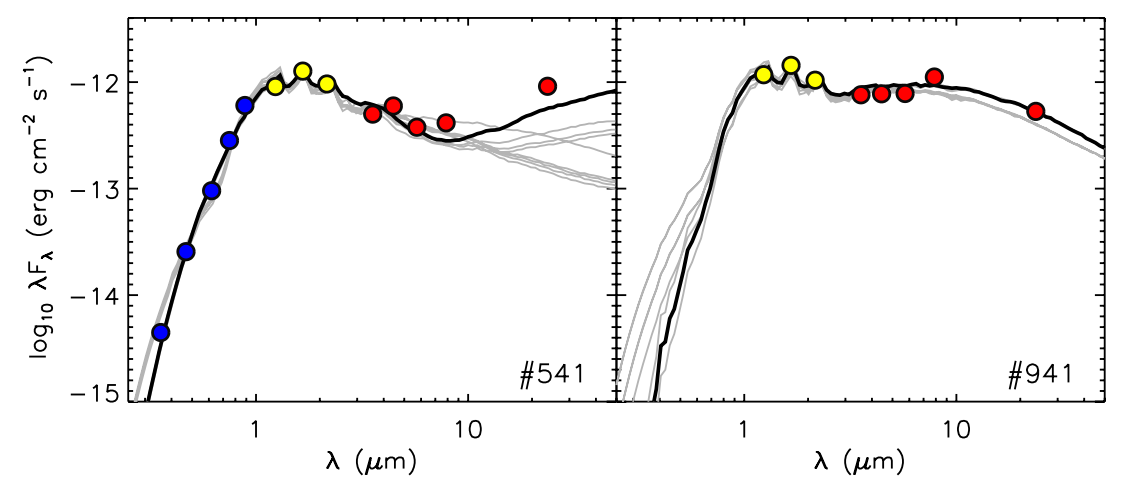

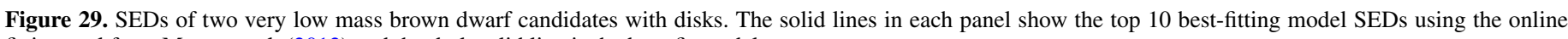
fitting tool from Mayne et al. (2012) and the dark solid line is the best-fit model.

(A color version of this figure is available in the online journal.)

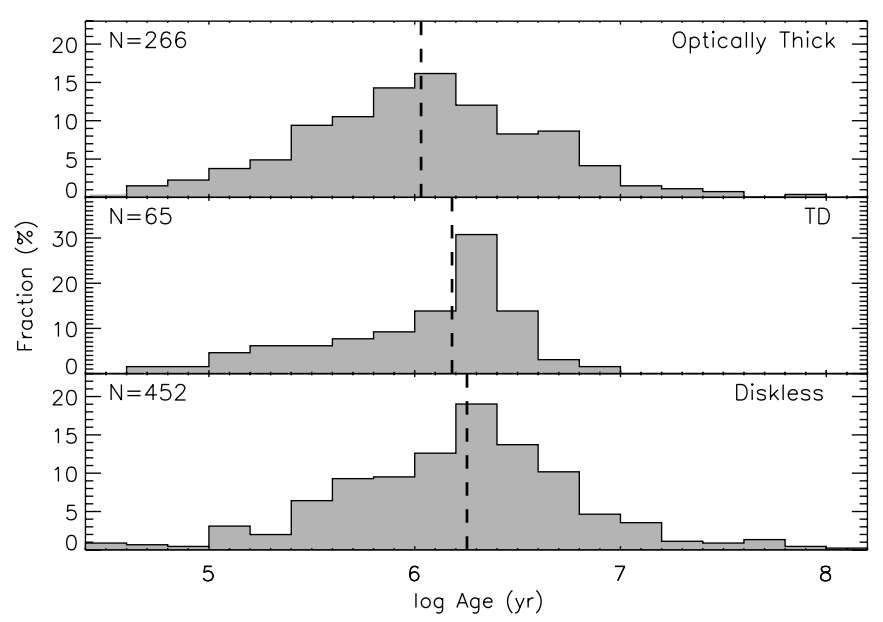

Figure 30. Histograms showing the age distribution for YSOs with optically thick disks, TDs, and without disks, respectively.

large inner holes, then there must be an efficient mechanism to dissipate the inner disks. In Figure 30, the age distribution of the TDs hints that there may be bimodality with one relatively flat distribution at age $\lesssim 1 \mathrm{Myr}$ and one peak at $\sim 2$ Myr. The two populations of TDs are more clearly distinguished in the H-R diagram (see Figure 10). The "young" TDs ( $<1 \mathrm{Myr}$ ) require a fast mechanism to dissipate the inner regions of their disks. One promising mechanism could be the interaction between the disk and a close binary (Lin \& Papaloizou 1993). The tidal interaction between the disk and a close companion can quickly clear away the material from the binary orbit, form a gap in the disks, and finally terminate the accretion from the outer disk to the inner disk (Lin \& Papaloizou 1993), thus accelerating the disk destruction process. Surveys toward nearby field G- and Mtype dwarfs have found about $50 \%$ of them have companions, with separation peaking at tens of AU (Fischer \& Marcy 1992). Furthermore, surveys toward star-forming regions have revealed the binary fraction to be even higher than in the field (Ghez et al. 1993, 1997; Leinert et al. 1993; Lafrenière et al. 2008). In general, such a high fraction of close binaries can explain these young diskless YSOs or TDs. However, the recent nearinfrared interferometric observations of five TDs in Taurus have excluded the possibility of having a companion with a flux ratio $\geqslant 0.05$, and a separation ranging from 0.35-4 AU (Pott et al. 2010). Alexander \& Armitage (2009) have proposed that young TD formation can be due to planet formation, while older ones could be formed due to photoevaporation, which works in disks

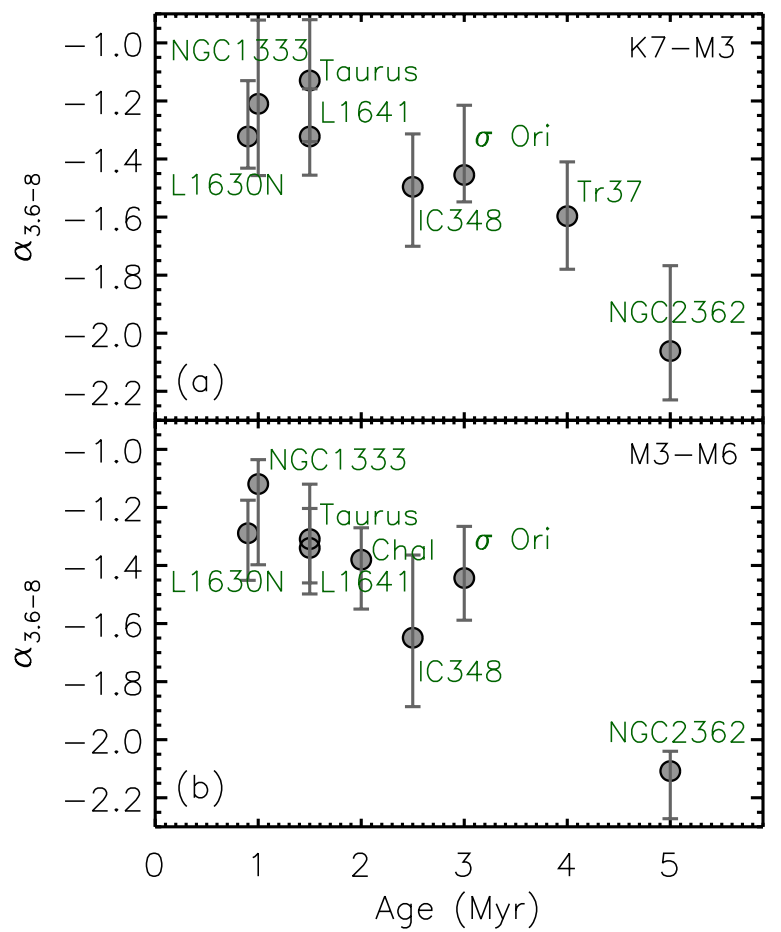

Figure 31. Mean spectral slope $\alpha_{3.6-8}$ of the disk population in different starforming regions vs. their ages. Panel (a) is for young stars with spectral types of $\mathrm{K} 7-\mathrm{M} 3$, and panel (b) is for young stars with spectral types of M3-M6. Cha I and $\operatorname{Tr} 37$ are missing from panels (a) and (b), respectively, due to the lack of members within those spectral-type ranges.

(A color version of this figure is available in the online journal.)

with substantial evolution. Sicilia-Aguilar et al. (2011) show that many TDs in the four-megayear-old cluster, $\operatorname{Tr} 37$, are due to grain evolution. Thus, a bimodal behavior in the age distribution of TDs is expected.

\subsubsection{The Evolution of the IRAC Spectral Slope}

In Figure 31, we show the average $\alpha_{3.6-8}$ of the disk population in several star-forming regions versus their ages. The regions include NGC 2068/2071 ( 1 Myr; Paper I), L1641 $(\sim 1.5 \mathrm{Myr}$; Paper I and this paper), Taurus $(\sim 1.5 \mathrm{Myr}$; Luhman et al. 2010), NGC 1333 ( 2 Myr; Winston et al. 2009, 2010), Cha I ( 2 Myr; Luhman et al. 2008), IC 348 ( 2.5 Myr; Lada et al. 2006; Muench et al. 2007), $\sigma$ Ori ( 3 Myr; Hernández et al. 2007b), Tr 37 ( 4 Myr; Sicilia-Aguilar et al. 2006a), and NGC 2362 ( 5 Myr; Currie et al. 2009). We separate the 


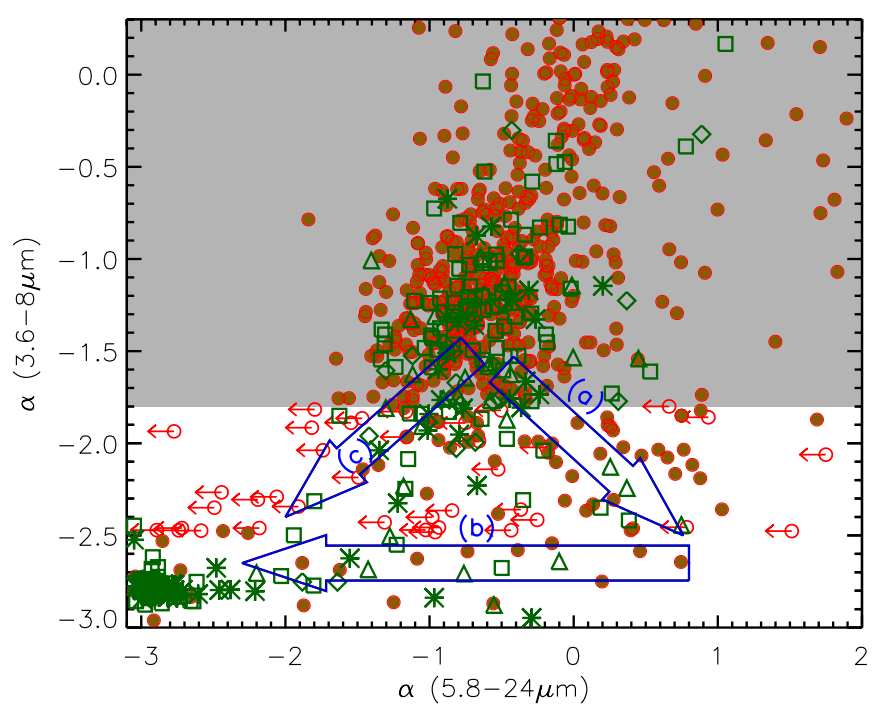

Figure 32. Spitzer infrared slopes of YSOs. The filled circles are the YSOs in L1641 with $24 \mu \mathrm{m}$ detections. The open circles with arrows are YSOs in L1641 without $24 \mu \mathrm{m}$ detections. The upper limits at $24 \mu \mathrm{m}$ were used to calculate $\alpha_{5.8-24}$. The YSOs in the relatively older clusters $\sigma$ Ori (open boxes), NGC 2362 (open triangles), Ori OB1 Association (open diamonds), and $\lambda$ Ori (asterisks) are also shown. The big arrows mark two possible paths for disk evolution. The arrows (a) and (b) show the path for radially depleted evolution, and the arrow (c) displays the path for globally depleted evolution. The disks that appear in the gray-shaded area harbor optically thick disks $\left(\alpha_{3.6-8}>-1.8\right)$.

(A color version of this figure is available in the online journal.)

disk population into two groups according to the spectral types of their central stars. For $\sigma$ Ori, NGC 2362, and NGC 1333, there are no available spectral types in the literature for most of their members and we use their $J$-band magnitude to estimate their spectral types using the isochrone at their corresponding ages. In Figure 31, a clear trend of $\alpha_{3.6-8}$ decreasing with age can be noted for each spectral type range, as noticed in SiciliaAguilar et al. (2006a). The average $\alpha_{3.6-8}$ decreases slowly over the first 3-4 Myr with most of the disk population harboring optically thick inner disks $\left(\alpha_{3.6-8}>-1.8\right)$. A fast decrease in the average $\alpha_{3.6-8}$ can be noted for the cluster NGC 2362 at an age of $\sim 5$ Myr.

\subsubsection{The Two Paths of Protoplanetary Disk Evolution}

Spitzer surveys of young clusters, such as IC 348, NGC 2362 , $\eta$ Cha, the Coronet cluster, and Tr 37 suggest that there are qualitatively two evolutionary paths for disks progressing from a primordial disk to a debris disk: (1) radially depleted disks and (2) globally depleted disks (Lada et al. 2006; Sicilia-Aguilar et al. 2008, 2009, 2011; Currie \& Kenyon 2009; Currie et al. 2009). Each path can be distinguished based on SEDs. The radially depleted disks show little or no excess emission in the shorter IRAC bands, but strong excess emission at $24 \mu \mathrm{m}$, suggesting that they are dissipating in an inside-out fashion. The globally depleted disks show more or less uniformly reduced infrared excess emission compared with primordial disks over all wavelengths out to $24 \mu \mathrm{m}$. This indicates that there is a reduction in their effective disk height for lack of small dust grains in disks (Currie \& Sicilia-Aguilar 2011; Sicilia-Aguilar et al. 2013). The reduced disk height can decrease the fraction of the stellar energy that is absorbed and reprocessed by the disk, which results in an SED with reduced infrared excess.

In Figure 32, we show the dereddened spectral slopes $\alpha_{3.6-8}$ and $\alpha_{5.8-24}$ for the YSOs in L1641. For the sources that are undetected at $24 \mu \mathrm{m}$, the $24 \mu \mathrm{m}$ upper limits are used to calculate $\alpha_{5.8-24}$. As a comparison, we also show the YSOs in several older clusters, $\sigma$ Ori $(\sim 3 \mathrm{Myr}$; Hernández et al. 2007b), NGC 2362 ( 5 Myr; Dahm \& Hillenbrand 2007), $\lambda$ Ori ( $~ 5$ Myr; Hernández et al. 2010), and Ori OB1 Association ( 5-10 Myr; Hernández et al. 2007a). As discussed above, the two disk evolution processes can be distinguished by their SEDs, which can be quantified by their spectral slopes. For the radially depleted disks, one would expect that $\alpha_{3.6-8}$ decreases while $\alpha_{5.8-24}$ increases due to the reduced fluxes at 5.8 and $8.0 \mu \mathrm{m}$. With the dissipation of the inner disk regions, $\alpha_{3.6-8}$ will be photospheric, and then as the outer disk is removed $\alpha_{5.8-24}$ starts to decrease. In globally depleted disks, $\alpha_{3.6-8}$ and $\alpha_{5.8-24}$ decrease simultaneously. In Figure 32 , the evolved disks $\left(\alpha_{3.6-8}<-1.8\right)$ are distributed around the triangle (marked by three arrows), which can be clearly explained by the two paths of disk evolution as described above.

\subsection{Accretion}

\subsubsection{Why are Accretion Rates Related to Stellar Masses?}

Figure 33 shows the relation between the mass accretion rate, derived from the $\mathrm{H} \alpha$ and $\mathrm{H} \beta$ line luminosities, and stellar mass for the YSOs in L1641, which is consistent with the $\dot{M}_{\text {acc }} \propto M_{*}^{\alpha}$ relation with $\alpha \sim 2-3$ as suggested in the literature (Calvet et al. 2004; Muzerolle et al. 2005; Mohanty et al. 2005; Natta et al. 2006; Garcia Lopez et al. 2006; Herczeg \& Hillenbrand 2008; Gatti et al. 2008). A large scatter in this relation is also noted. Figure 33 also shows the $\dot{M}_{\text {acc }}$ versus $M_{*}$ relation for $\sim 20$ TDs. These objects generally follow the same trend as the normal CTTSs. Furthermore, TDs in Figure 33(b) seem to show slightly lower accretion rates than the normal disk populations at the same stellar masses, which contradicts those in Figure 33(a). To understand this phenomenon, we need to distinguish the better tracer for accretion from $\mathrm{H} \alpha$ and $\mathrm{H} \beta$, which is beyond the scope of this paper.

In Figure 34, we present another $\dot{M}_{\text {acc }}$ versus $M_{*}$ plot for the YSOs in L1641. The accretion rates here are estimated from the $\mathrm{FW}_{\mathrm{H} \alpha, 10 \%}$ of the high-resolution $\mathrm{H} \alpha$ line profiles in the Hectochelle spectra. Similar to the result in Figure 33 and in the literature, the accretion rates generally increase with stellar masses with a large scatter in $\dot{M}_{\text {acc }}$ at similar masses. We divided the YSOs into four mass bins, and estimated the median accretion rates in each mass bin as in Rigliaco et al. (2011a). For comparison we show the median accretion rates within the same mass bins for YSOs in $\rho$ Oph and $\sigma$ Ori (Rigliaco et al. 2011a). The L1641 region has an age between the $\rho$ Oph $(<1$ Myr $)$ and $\sigma$ Ori $(\sim 3 \mathrm{Myr})$ regions. Within each mass bin, the median accretion rate in $\rho$ Oph is higher than that in $\sigma$ Ori, indicating a trend of decreasing accretion rate with age. The YSOs in L1641 show median accretion rates similar to those in $\rho$ Oph within the bins for masses $>0.5 M_{\odot}$, and similar to those in $\sigma$ Ori within the bins with masses $<0.5 M_{\odot}$, which may indicate that the accretion rate decreases faster with age in the low-mass range than in the high-mass range.

Hartmann et al. (2006) discussed the angular momentum transport in disks. MRI (Balbus \& Hawley 1998) is the key mechanism for angular momentum transport. For MRI to take effect, ionization in the disks is required. There are different sources that can ionize the gas in disks, including cosmic rays or stellar UV and X-ray photons impinging on the disk surface (Gammie 1996; Glassgold et al. 1997), and thermal ionization driven by either viscous dissipation within the disk or irradiation 

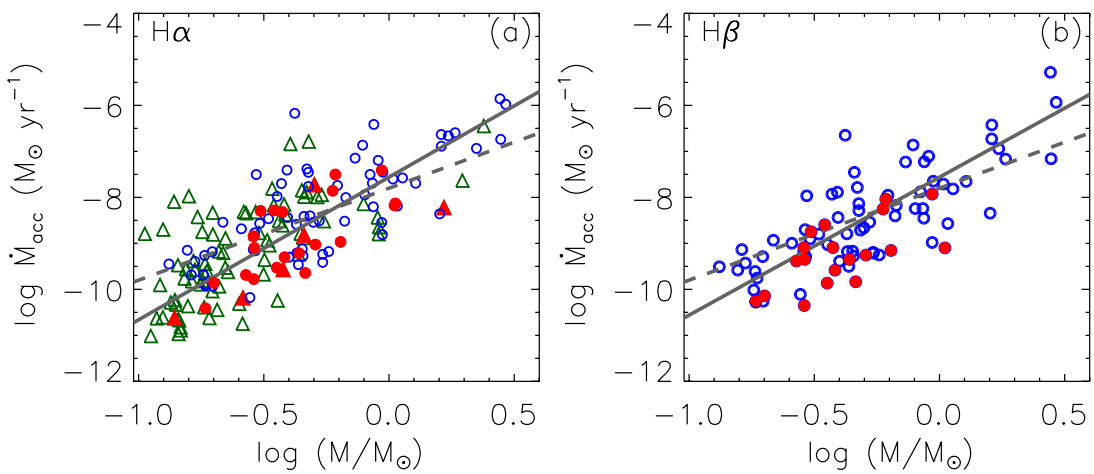

Figure 33. Relation between accretion rates deduced from the $\mathrm{H} \alpha(\mathrm{a})$ and $\mathrm{H} \beta(\mathrm{b})$ line luminosities and stellar mass for the CTTSs in this paper. In panel (a), the (open and filled) circles are for the YSOs in our Hectospec survey, and the (open and filled) triangles are for the YSOs using the data from Hsu et al. (2012). The filled circles and triangles are for TDs. In panel (b), the symbols are the same as in panel (a). The slopes for the $\dot{M}_{\text {acc }} \propto M_{*}^{2}$ relation (the dashed line) and for the $\dot{M}_{\text {acc }} \propto M_{*}^{3}$ relation (the solid line) are presented. Note that these lines are merely indicative and not fits to the data in each panel.

(A color version of this figure is available in the online journal.)

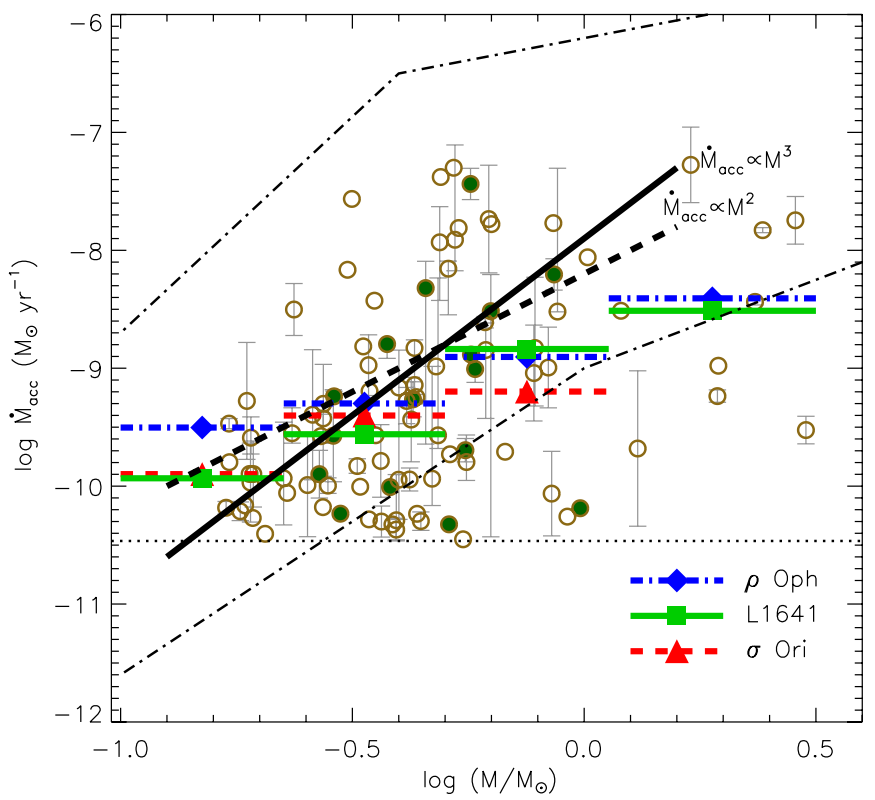

Figure 34. Relation between accretion rates deduced from the $\mathrm{FW}_{\mathrm{H} \alpha, 10 \%}$ of the $\mathrm{H} \alpha$ line profiles and stellar mass. The filled circles are for the TDs. The open circles represent the other disk population in L1641. The sources are considered accretors when $\mathrm{FW}_{\mathrm{H} \alpha, 10 \%}>250 \mathrm{~km} \mathrm{~s}^{-1}$, which corresponds to an accretion rate of $3.4 \times 10^{-11} M_{\odot} \mathrm{yr}^{-1}$ marked with the dotted line in the figure. For the sources with multiple observations, the median accretion rates are used for the plot with error bars showing their minimum and maximum accretion rates. The YSOs are divided into four mass bins. The thick solid lines centered on filled boxes show the median accretion rate within these mass bins. As a comparison, the median accretion rates for YSOs in $\rho$ Oph and $\sigma$ Ori are shown as the thick dash-dotted lines centered on filled diamonds, and dashed lines centered on filled triangles, respectively (Rigliaco et al. 2011a). The slopes for the $\dot{M}_{\text {acc }} \propto M_{*}^{2}$ relation (the dark dash line), and for $\dot{M}_{\text {acc }} \propto M_{*}^{3}$ relation (the dark solid line) are presented. The dash-dotted lines show the accretion rate detection limits for the T Tauri stars in the literature (see Paper I).

(A color version of this figure is available in the online journal.)

of the disk surface by the central star (Hartmann et al. 2006). Hartmann et al. (2006) considered the two limiting cases: (1) a lower-mass disk, in which the entire column is ionized for active MRI, which is subject to full viscous evolution, resulting in $\alpha \approx 2.5$; and (2) a higher-mass disk, in which thermal ionization by irradiation from the central star is dominant, causing an MRI-active surface layer resulting in $\alpha \approx 1$. To test this scenario, we have collected a large set of YSOs with accretion rate estimates from the literature (Gullbring

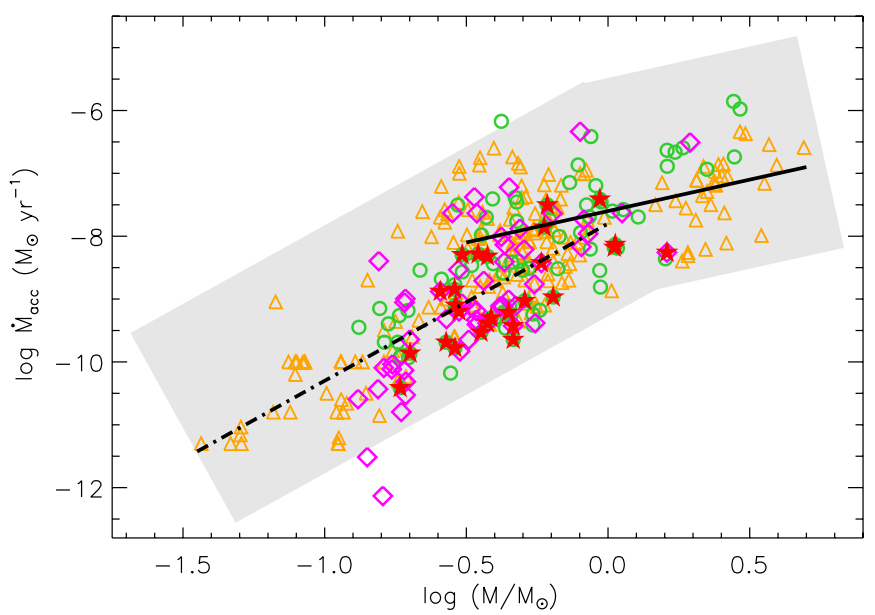

Figure 35. Relation between accretion rate from $\mathrm{H} \beta$ emission line luminosity and stellar mass for the CTTSs in this work (open circles), Paper I (open diamonds), and other literature (open triangles). The filled star symbols are the TDs in L1641 and L1630N. We also include the $\dot{M}_{\text {acc }} \propto M_{*}$ relation (black solid line) and the $\dot{M}_{\text {acc }} \propto M_{*}^{2.5}$ relation (black dash-dotted line). Note that these lines are merely indicative and not fits to the data in each panel.

(A color version of this figure is available in the online journal.)

et al. 1998; White \& Basri 2003; Muzerolle et al. 2003, 2005; Hartigan \& Kenyon 2003; Calvet et al. 2004; Natta et al. 2006; Mohanty et al. 2005; Garcia Lopez et al. 2006; Herczeg \& Hillenbrand 2008; Dahm 2008; Paper I), within the star-forming regions Taurus-Aurigae, IC 348, $\rho$ Oph, $\lambda$ Ori, Orion OB, upper Scorpius, and L1641, and L1630N. All these data are shown in Figure 35. The dependency of $\dot{M}_{\text {acc }}$ on $M_{\star}$ is steeper for the low-mass disks around low-mass stars and brown dwarfs than for higher mass disks around solar- to intermediate-mass stars, which may support the scenario proposed by Hartmann et al. (2006). However, given the large scatter in Figure 35, the difference on the dependency of $M_{\text {acc }}$ on $M_{\star}$ in different mass ranges is only marginally significant. Furthermore, it is possible that the ages of intermediate-mass stars are older than the sub-solar mass stars in Figure 35. However, there is a large uncertainty in estimating ages of intermediate-mass stars from the H-R diagram (Hartmann 1999), which complicates the comparison. 


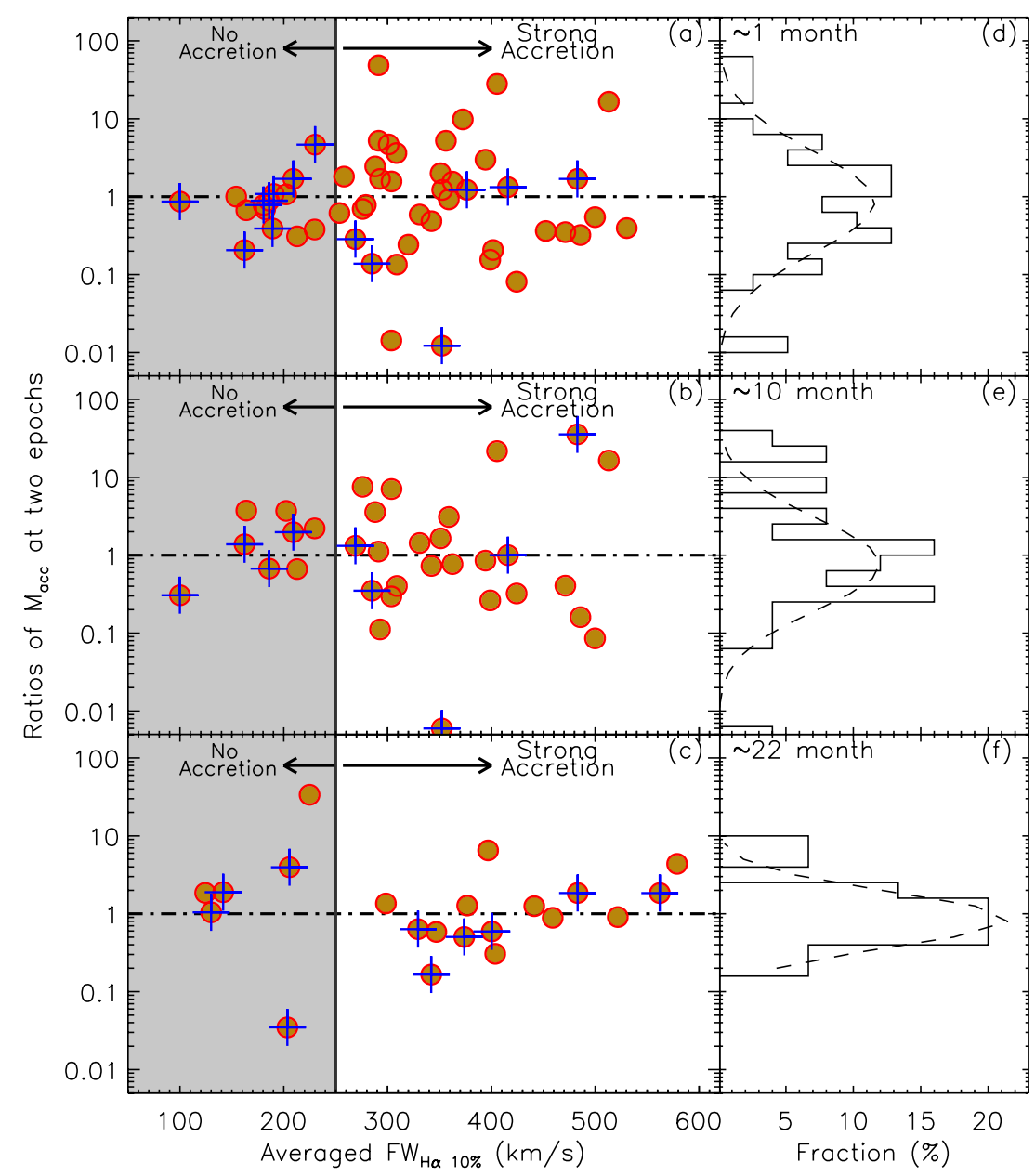

Figure 36. Left panels: the ratio of accretion rates $\left(\dot{M}_{\text {acc }}\right)$ observed at two epochs separated by $\sim 1,10$, and 22 months vs. averaged $\mathrm{FW}_{\mathrm{H} \alpha, 10 \%}$ for the disk population

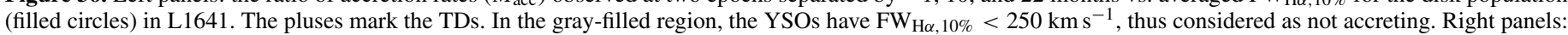
the distribution of ratios of $\dot{M}_{\text {acc }}$ for the accretors $\left(\mathrm{FW}_{\mathrm{H} \alpha, 10 \%}>250 \mathrm{~km} \mathrm{~s}^{-1}\right)$ shown in the left panels. The dashed line shows the fit to the distribution using the Gaussian function with a standard deviation $\sim 0.6$ for (d) and (e), and $\sim 0.3$ for (f).

(A color version of this figure is available in the online journal.)

\subsubsection{Can Accretion Variability Explain the Scattering in $\dot{M}_{\text {acc }}$ versus $M_{*}$ ?}

Though the correlation between accretion rate and stellar mass is obvious for a large sample of young stars, individual objects with similar ages and masses commonly scatter around the average relation by up to two orders of magnitude. Observations show that the amplitude of accretion variation for CTTSs is large (Johns \& Basri 1995; Gullbring et al. 1996; Alencar et al. 2001; Alencar \& Batalha 2002; Sicilia-Aguilar et al. 2010). Thus, the temporal accretion variation is proposed as one potential explanation for the large scattering in the $\dot{M}_{\text {acc }}$ versus $M_{*}$ plot. To test this, we have performed a multi-epoch highresolution spectroscopic survey of YSOs with Hectochelle covering baselines of $\sim 1,10$, and $\sim 22$ months. A total of 52 disked YSOs were observed at two epochs separated by an interval of $\sim 1$ month, 33 YSOs were observed at two epochs separated by $\sim 10$ months, and 21 YSOs were observed at two epochs separated by $\sim 22$ months. Figures 36 (a)-(c) show the ratio of accretion rates $\left(\dot{M}_{\text {acc }}\right)$ estimated from $\mathrm{FW}_{\mathrm{H} \alpha, 10 \%}$ at epochs separated by $\sim 1,10$, and 22 months, respectively, against their averaged $\mathrm{FW}_{\mathrm{H} \alpha, 10 \%}$. The accretion rates are estimated from $\mathrm{FW}_{\mathrm{H} \alpha, 10 \%}$ via the relation between the accretion rate and $\mathrm{FW}_{\mathrm{H} \alpha, 10 \%}$ from Natta et al. (2004). Figures 36(d)-(f) show the distribution of accretion rate ratios for the accreting YSOs $\left(\mathrm{FW}_{\mathrm{H} \alpha, 10 \%}>250 \mathrm{~km} \mathrm{~s}^{-1}\right)$ which are shown in left panels of Figure 36, respectively. The Gaussian fits to these distributions have standard deviations in the logarithm of the accretion rate ratio of $\sim 0.66 \pm 0.09,0.59 \pm$ 0.12 , and $0.35 \pm 0.06$, respectively. The small standard deviation for the twenty-two-month interval could be due to the low statistics. Thus, our data suggest that the accretion variability cannot explain the two orders of magnitude scatter in accretion rates at similar masses. Our result confirms the finding in Nguyen et al. (2009b) and Sicilia-Aguilar et al. (2010). Nguyen et al. (2009b) have investigated the accretion variability toward a sample of 40 young stars in Taurus and Cha I based on the Ca II fluxes, and concluded that the intrinsic source variability cannot be the dominating factor in the large scatter of the $\dot{M}_{\text {acc }}$ versus $M_{*}$ relationship.

The uncertainties in calculating $\dot{M}_{\text {acc }}$ can induce a scatter of the $\dot{M}_{\text {acc }}$ versus $M_{*}$ relationship. Herczeg \& Hillenbrand (2008) discuss the possible uncertainty contributors, including distance, stellar parameters (extinction, effective temperature, mass, and radius), assumption of accretion geometry, and conversion from UV excess continuum emission to accretion luminosity. They concluded that these uncertainties can lead to a $\sim 0.6 \mathrm{dex}$ uncertainty in $\dot{M}_{\text {acc }}$ for calculating $\dot{M}_{\text {acc }}$ of young stars in Taurus from UV excess continuum emission. When $\dot{M}_{\text {acc }}$ is calculated from emission lines, the uncertainties $(\sim 0.8-1.0 \mathrm{dex})$ in $\dot{M}_{\text {acc }}$ 


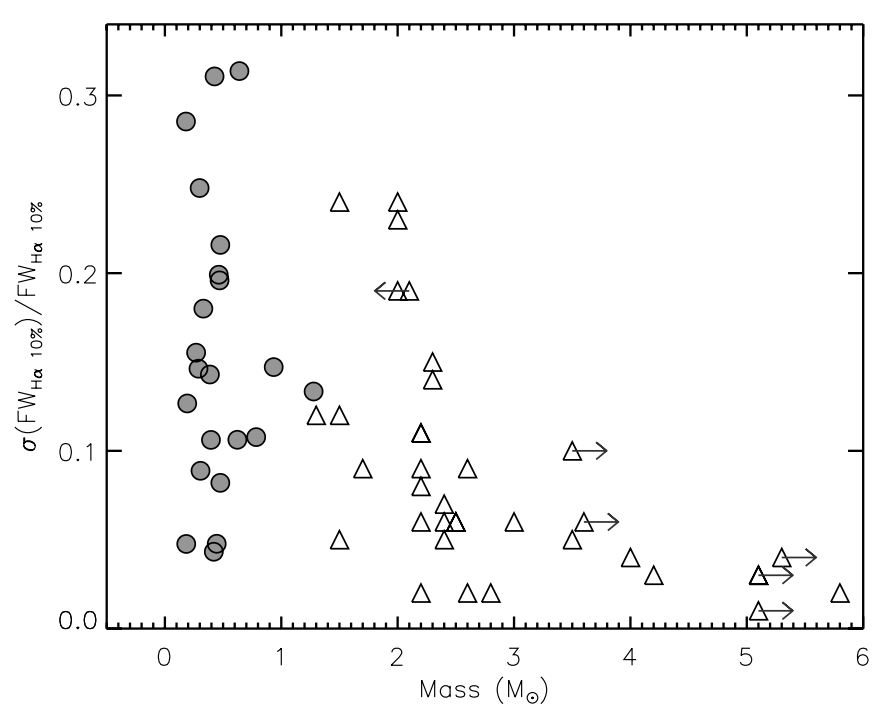

Figure 37. Relative variability of $\mathrm{FW}_{\mathrm{H} \alpha, 10 \%}$ vs. stellar mass. The filled circles show the disk population with three-epoch Hectochelle data. The triangles present the sources from Mendigutía et al. (2011). The left pointing and right pointing arrows mark the lower and upper limits for the stellar masses.

could be bigger than those from UV excess emission due to large uncertainties in the conversions of line quantities (line luminosities or $\left.\mathrm{FW}_{\mathrm{H} \alpha, 10 \%}\right)$ to accretion quantities $\left(L_{\mathrm{acc}}\right.$ or $\left.\dot{M}_{\mathrm{acc}}\right)$, which are constructed mostly based on non-simultaneous data (Paper I; Muzerolle et al. 1998; Natta et al. 2004; Herczeg \& Hillenbrand 2008; Rigliaco et al. 2012). The combination of uncertainty (0.6-1.0 dex) in measurement and accretion variability $(0.5$ dex $)$ may account for the large scatter in the $\dot{M}_{\text {acc }}$ versus $M_{*}$ relationship.

We estimate the relative variability of $\mathrm{FW}_{\mathrm{H} \alpha, 10 \%}$ $\left(\sigma /\left\langle\mathrm{FW}_{\mathrm{H} \alpha, 10 \%}\right\rangle\right)$ for the disk population with three-epoch Hectochelle data and plot this against stellar masses in Figure 37. As a comparison, we also show a sample of intermediate-mass stars collected from Mendigutía et al. (2011). In the figure, the relative variability of $\mathrm{FW}_{\mathrm{H} \alpha, 10 \%}$ shows a large scatter for stars less than $3 M_{\odot}$, and the scatter increases with decreasing mass. The values of $\sigma /\left\langle\mathrm{FW}_{\mathrm{H} \alpha, 10 \%}\right\rangle$ are much smaller and show less scatter for the Herbig Be stars $\left(M_{*}>3 M_{\odot}\right)$. Mendigutía et al. (2011) suggest that the distinction between $\sigma /\left\langle\mathrm{FW}_{\mathrm{H} \alpha, 10 \%}\right\rangle$ for Herbig Ae stars and Herbig Be stars is due to the different formation mechanisms of the $\mathrm{H} \alpha$ emission line. For Herbig Ae stars, $\mathrm{H} \alpha$ line fluxes may partially originate from the magnetospheric accretion flow, and thus are sensitive to accretion variations. In Herbig Be stars, $\mathrm{H} \alpha$ emission may come from the disk surface (Vink et al. 2002; Mottram et al. 2007) and thus be less sensitive to accretion variations. In this work, we extend the study of Mendigutía et al. (2011) to the sub-solar mass regime, and find that the mean $\sigma /\left\langle\mathrm{FW}_{\mathrm{H} \alpha, 10 \%}\right\rangle$ in the low-mass range is larger than that of Herbig Ae stars. This may be due to two factors: (1) the accretion of sub-solar mass stars is more variable than Herbig Ae stars or (2) accretion rate variability is independent of mass, but the $\mathrm{H} \alpha$ emission line in Herbig Ae stars includes a contribution from a disk wind, which is less susceptible to accretion rate fluctuations, diluting the fluctuations in the $\mathrm{H} \alpha$ line.

\subsubsection{How Does Accretion Work in Disks at Different Evolutionary Stages?}

In Figure 38(a), we compare the $\mathrm{H} \alpha$ EWs of our TDs with those of the YSOs with optically thick disks $\left(\alpha_{3.6-8} \geqslant-1.8\right)$ and
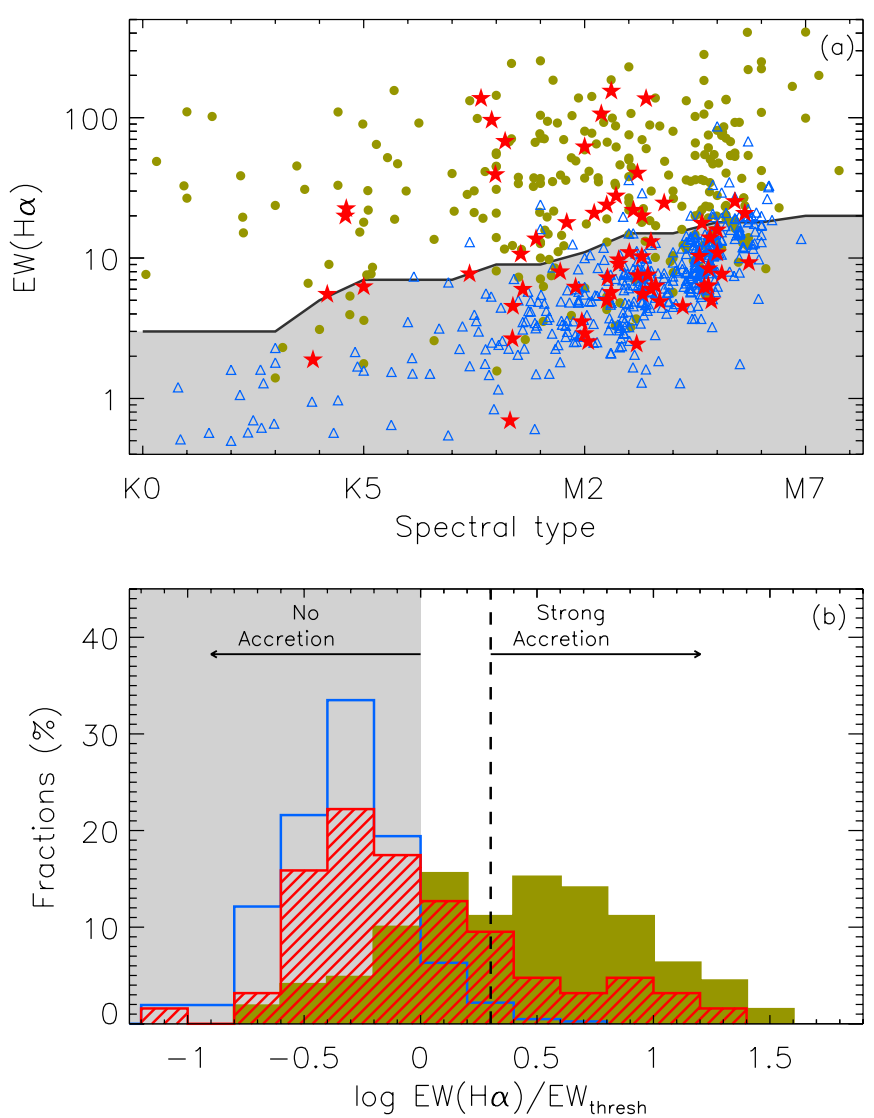

Figure 38. (a) H $\alpha$ EWs vs. spectral type for known YSOs in L1641. The filled circles are for YSOs with optically thick disks and triangles are for YSOs without disks (in this work and Paper I). The stars show the TDs in L1641. (b) The distribution of the ratio between the observed $\mathrm{H} \alpha \mathrm{EW}$ and the EW threshold, separating CTTSs or WTTSs, for the three populations in the left panel: YSOs with optically thick disks (filled histogram), YSOs without disks (open histogram), and TDs (line-filled histogram). The dash line marks $\mathrm{EW}(\mathrm{H} \alpha) / \mathrm{EW}_{\text {thresh }}=2$.

(A color version of this figure is available in the online journal.)

diskless PMS stars. The YSOs with optically thick disks usually show strong $\mathrm{H} \alpha$ emission, while the PMS stars without disks present weak $\mathrm{H} \alpha$ emission. The TDs straddle this boundary with a major fraction of TDs showing no active accretion and a fraction of TDs showing strong accretion activity. In Figure 38(b), we show the distribution of the logarithmic ratio between the observed $\mathrm{H} \alpha \mathrm{EW}$ and the EW threshold for the three populations in Figure 38(a). Here, the EW threshold is the spectral-type-dependent threshold used to classify the YSOs into CTTSs or WTTSs (see Paper I). Using these EW thresholds, $79 \% \pm 5 \%$ of YSOs with optically thick disks are classified as CTTSs while only $40 \% \pm 8 \%$ of TDs are CTTSs. And $21 \% \pm$ $6 \%$ of TDs show "strong" accretion activity, defined as having $\mathrm{H} \alpha \mathrm{EW}$ of more than two times the EW thresholds. For the YSOs with optically thick disks, this fraction is $60 \% \pm 5 \%$. Therefore, there are significantly fewer strong accretors among TDs compared with optically thick disks. For the six globally depleted disks shown in Figure 16, only source 16 shows an $\mathrm{H} \alpha$ $\mathrm{EW}$, which is marginally above the threshold for classifying it as a CTTS. The active accretion in source 16 is confirmed by the $\mathrm{H} \alpha$ emission line profile $\left(\mathrm{FW}_{\mathrm{H} \alpha, 10 \%} \sim 387 \mathrm{~km} \mathrm{~s}^{-1}\right)$ observed with Hectochelle (see Figure 23). Thus, statistically, only 1/6 of YSOs with globally depleted disks are CTTSs, which is much lower than the YSOs with optically thick disks or TDs. 


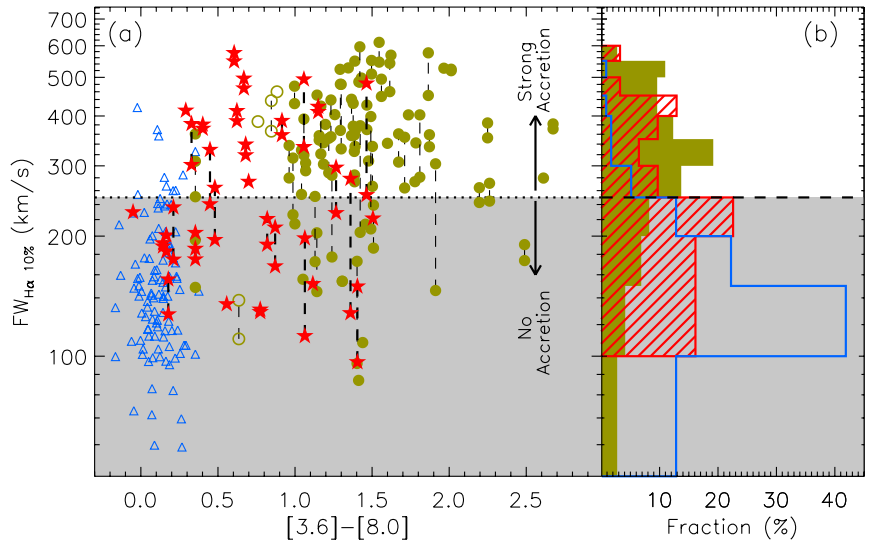

Figure 39. (a) $\mathrm{FW}_{\mathrm{H} \alpha, 10 \%}$ vs. [3.6] - [8.0] colors for YSOs in L1641. The open triangles show the sources without disks, the open circles display the YSOs with optically thin disks $\left(\alpha_{3.6-8}<-1.8\right)$, and the filled circles are for YSOs with optically thick disks $\left(\alpha_{3.6-8} \geqslant-1.8\right)$. The stars show the TDs in L1641. A set of two symbols connected by a dashed line are the minimum and maximum $\mathrm{FW}_{\mathrm{H} \alpha, 10 \%}$ when there are multi-epoch observations. (b) The distribution of $\mathrm{FW}_{\mathrm{H} \alpha, 10 \%}$ for the diskless population (open histograms), the optically thick disk population (filled histograms), and the TDs (line-filled histograms) in L1641. For the YSOs with two or three observations, the average $\mathrm{FW}_{\mathrm{H} \alpha, 10 \%}$ is used to derive these distributions. In the gray-filled region, the YSOs have $\mathrm{FW}_{\mathrm{H} \alpha, 10 \%}<250 \mathrm{~km} \mathrm{~s}^{-1}$; thus they are considered not accreting.

(A color version of this figure is available in the online journal.)

In Figure 39(a), we show the $\mathrm{FW}_{\mathrm{H} \alpha, 10 \%}$ versus [3.6] - [8.0] colors for the YSOs in L1641. The diskless stars typically show small $\mathrm{FW}_{\mathrm{H} \alpha, 10 \%}$, with $90 \% \pm 9 \%$ of them showing $\mathrm{FW}_{\mathrm{H} \alpha, 10 \%}<250 \mathrm{~km} \mathrm{~s}^{-1}$. The diskless stars in L1641 show a $\mathrm{FW}_{\mathrm{H} \alpha, 10 \%}$ distribution, peaked at $\sim 125 \mathrm{~km} \mathrm{~s}^{-1}$, which is similar to that of diskless stars collected in the literature (see Appendix A). The optically thick disk population has typically large $\mathrm{FW}_{\mathrm{H} \alpha, 10 \%}$ with a smaller number of sources showing $\mathrm{FW}_{\mathrm{H} \alpha, 10 \%}<250 \mathrm{~km} \mathrm{~s}^{-1}$. The TDs show a broader and flatter distribution of $\mathrm{FW}_{\mathrm{H} \alpha, 10 \%}$ compared with the other two populations. If using $\mathrm{FW}_{\mathrm{H} \alpha, 10 \%}>250 \mathrm{~km} \mathrm{~s}^{-1}$ as the criterion for active accretion, then $45 \% \pm 12 \%$ of TDs are accretors. The fraction of accretors increases to $77 \% \pm 10 \%$ among the optically thick disk population. The fractions of accretors for the two populations identified with $\mathrm{FW}_{\mathrm{H} \alpha, 10 \%}$ are consistent with those $(40 \% \pm 8 \%$ versus $79 \% \pm 5 \%)$ based on the $\mathrm{H} \alpha$ EWs. Though the fraction of accretors among TDs is significantly lower than that in the optically thick disk population, the accretors in both populations show similar median values of $\mathrm{FW}_{\mathrm{H} \alpha, 10 \%}$, which are 387 and $375 \mathrm{~km} \mathrm{~s}^{-1}$, respectively, thus corresponding to similar accretion rates (Natta et al. 2004). This finding confirms our previous result in Paper I. Sicilia-Aguilar et al. (2010) estimated the accretion rates of a sample of YSOs in the $\operatorname{Tr} 37$ cluster using the $U$-band excess method, and also found that the median accretion rates of the accreting TDs and normal CTTSs are similar. This result is inconsistent with what Najita et al. (2007) found in Taurus, namely, that the accretion rates of TDs are $\sim 10$ times lower than those of normal accreting $\mathrm{T}$ Tauri stars at the same disk mass. Recently, Espaillat et al. (2012) found that the median accretion rate of full disks is $\sim 4$ times higher than that of accreting TDs in NGC 2068 and IC 348 . Thus, the relation between accretion processes in young stars and the age or evolutionary stage of their disks is still controversial, and may vary in different star-forming regions.

In this work, we use the $\mathrm{H} \alpha$ line as a tracer of accretion. The criteria that we use to classify CTTS and WTTS may affect our results. In Figure 39, we consider the YSOs with

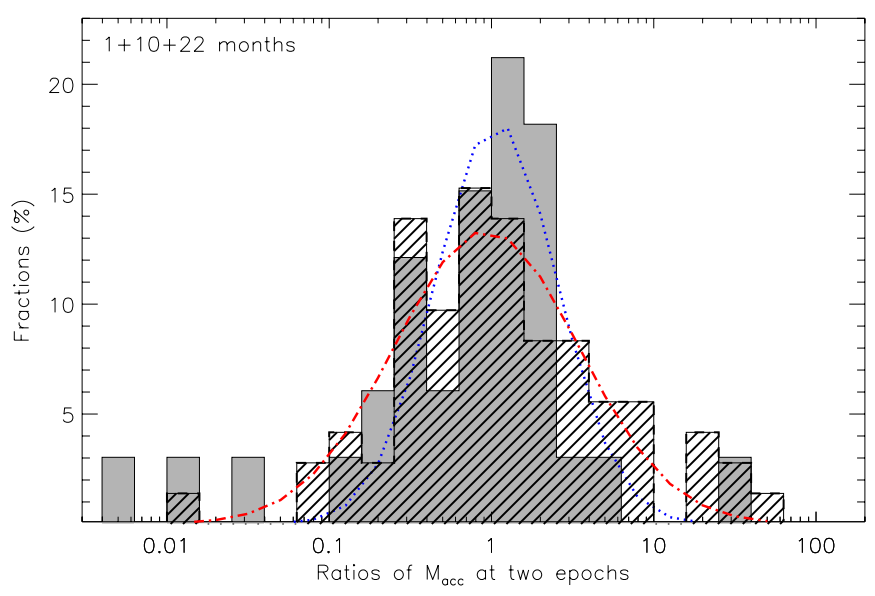

Figure 40. Distribution of ratios of $\dot{M}_{\text {acc }}$ for TDs (filled histograms) and normal disk population (line-filled histograms) with observations at two epochs separated by $\sim 1,10$, or 22 months. The dash-dotted line shows the Gaussian fit to the logarithm of the ratio for the normal disk population with $\sigma \sim 0.56$. The dotted line shows the Gaussian fit to the distribution of TDs with $\sigma \sim 0.37$.

(A color version of this figure is available in the online journal.)

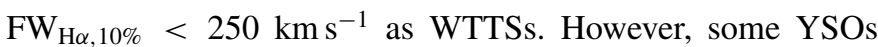
with tiny accretion rates or with infall perpendicular to the line of sight may show $\mathrm{FW}_{\mathrm{H} \alpha, 10 \%}<250 \mathrm{~km} \mathrm{~s}^{-1}$. We could exclude more of these YSOs among the TDs than those among the optically thick disks since more than half of TDs show $\mathrm{FW}_{\mathrm{H} \alpha, 10 \%}<250 \mathrm{~km} \mathrm{~s}^{-1}$, but only $20 \%$ of the optically thick disk population have $\mathrm{FW}_{\mathrm{H} \alpha, 10 \%}<250 \mathrm{~km} \mathrm{~s}^{-1}$ (see the above discussion). If we change the criterion of $\mathrm{FW}_{\mathrm{H} \alpha, 10 \%}$ to $200 \mathrm{~km} \mathrm{~s}^{-1}$ for classifying CTTS and CTTS, then the median values of $\mathrm{FW}_{\mathrm{H} \alpha, 10 \%}$ for accreting TDs and the optically thick disk population are 329 and $356 \mathrm{~km} \mathrm{~s}^{-1}$, respectively, and the corresponding accretion rate for TDs is $\sim 2$ times lower than that of the optically thick disks, which can slightly reconcile the contradiction between our results and the others in the literature.

In Figure 40, we combine the three distributions (the distribution of ratios of $\dot{M}_{\text {acc }}$ ) shown in Figures 36(d)-(f), and separate that into two distributions, one of which is for TDs and the other for the optically thick disk population $\left(\alpha_{3.6-8}>-1.8\right)$. We use the Gaussian function to fit the distribution in the logarithm of the ratio, and derive standard deviations of $\sim 0.37 \pm 0.05$ and $0.56 \pm 0.07$, respectively, suggesting that there is no significant difference between the accretion variability of TDs and normal disks. We have already found that the accreting TDs and normal disk population show similar median accretion rates. Here, we find their accretion variability is also similar. Thus, we conclude that the accretion activity of accreting TDs is similar to the normal disk population.

\subsection{Star Formation Modes in L1641}

With the data from Spitzer, XMM, and the spectroscopic survey, we have performed a census of YSOs in L1641 (see Tables 2 and 3). Based on this YSO catalog, we re-examine the issue of the star formation mode in L1641 (i.e., clustered versus isolated). We estimated the surface density (SD) using the nearest neighbor method, which is given with the equation density $=n /\left(\pi r_{n}^{2}\right)$, where $r_{n}$ is the distance to the $n$th nearest YSO and $n$ is set to 10. We calculate the SD for all cataloged YSOs ( $\sim 1390$ sources) in Tables 2 and 3. We also calculate the SD for known YSOs in Taurus (Luhman et al. 2010) and IC 348 (Muench et al. 2007). Their SD distributions are shown in 


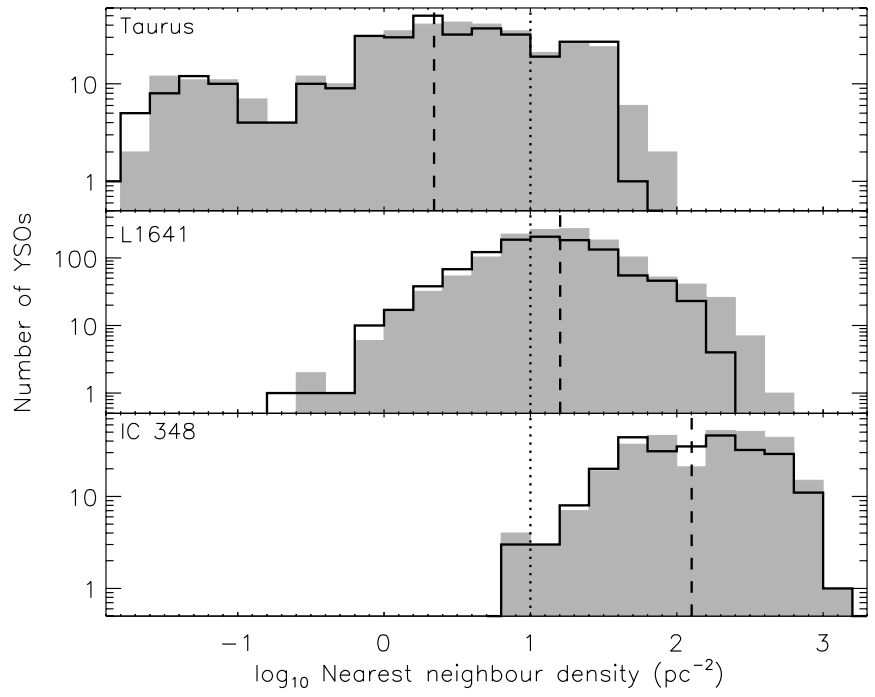

Figure 41. Distribution of YSO nearest neighbor densities for Taurus, L1641, and IC 348. The densities are calculated for each YSO using the equation density $=n /\left(\pi r_{n}^{2}\right)$, where $r_{n}$ is the distance to the $n$th nearest YSO and $n$ is set to 10. In each panel, the gray-filled histograms include all known YSOs in each region, while the open histograms only contain the YSOs with masses larger than $\sim 0.05 M_{\odot}$. In each panel, the dotted line marks the density of $10 \mathrm{pc}^{-2}$, and the dashed line shows the median values for the open histograms in each region.

Figure 41. The median SD is 2.8, 15.7, and $177.5 \mathrm{pc}^{-2}$ in Taurus, L1641, and IC 348, respectively. To allow a comparative study of the SD distributions in these regions, we need to calculate the SD for a selected sample of YSOs in each region down to a similar completeness limit. In L1641, we only select the YSOs with $K_{\mathrm{s}}<14.3(10 \sigma$ limit in the 2MASS survey), corresponding to a $0.05 M_{\odot}$ PMS star at the median age of L1641 (Baraffe et al. 1998). We use this sample mass limit to select the YSOs in Taurus and IC 348. We calculate the SDs for the selected YSOs in each region, and show their distributions in Figure 41. The median SDs are 2.6, 12.5, and $141.4 \mathrm{pc}^{-2}$ in Taurus, L1641, and IC 348, respectively. The result is consistent with the common sense that the star formation mode in Taurus is in isolation, and in IC 348 is in cluster. Among the selected YSO sample in each region, $79 \%$ of the YSOs in Taurus are located in regions with an SD less than $10 \mathrm{pc}^{-2}$, while in IC 348 the fraction is only $1 \%$. In L1641, $41 \%$ of YSOs live in regions with $\mathrm{SD}<10 \mathrm{pc}^{-2}$. In addition, in L1641, only $6 \%$ of YSOs are in areas with SD less than $2.6 \mathrm{pc}^{-2}$ (the median SD in Taurus), and only 5\%o of YSOs are in areas with SD larger than $141.4 \mathrm{pc}^{-2}$ (the median $\mathrm{SD}$ in IC 348). The distribution of SDs in the L1641 cloud is between that of Taurus and IC 348, confirming that both of the two star formation modes (isolated and clustered) are at work in L1641 as suggested in the literature (Strom et al. 1993; Allen 1995; Paper I).

We estimate the SDs for Class III, Class II, and flatspectrum+Class I sources in L1641. The median SDs are $14.7 \mathrm{pc}^{-2}, 17.2 \mathrm{pc}^{-2}$, and $17.7 \mathrm{pc}^{-2}$, for Class III, Class II, and flat-spectrum/Class I sources, respectively. In addition, $32 \%$ of Class III sources, $28 \%$ of Class II sources, and $32 \%$ of flatspectrum/Class I sources are located in regions with SDs less than $10 \mathrm{pc}^{-2}$. Thus, our result suggests there is no significant difference in the SDs between the "old" Class III sources and "young" Class II or flat-spectrum/Class I sources.

We compare the ages of "isolated" YSOs $\left(\mathrm{SD} \leqslant 10 \mathrm{pc}^{-2}\right)$ with those of "clustered" YSOs (SD > $\left.10 \mathrm{pc}^{-2}\right)$ in L1641 using the sample young stars with spectral types. Both populations show very broad age distributions with median ages of 1.6 and 1.5 Myr, respectively. A K-S test suggests that the age distribution of both populations drawn from the same distribution has a probability of $\sim 0.3$.

\subsection{Star Formation History in L1641, and the Lifetime of Flat-spectrum and Class I Sources}

In this work and Paper I, we have more than 800 young stars with spectral-type estimates. For these YSOs, we can derive their ages from the PMS evolutionary tracks and statistically investigate the SFH in L1641. In Figure 42, we show the distribution of ages for all these YSOs estimated from four sets of PMS evolutionary tracks. The age distributions from D08 and DM97 suggest the formation rate of stars began to accelerate in L1641 10 Myr ago. The age distributions estimated from D08 and DM97 can be fit using the following formula:

$$
\log N \propto \kappa \times \tau
$$

where $N$ is the number of YSOs and $\tau$ is the age in units of Myr. Fitting to the YSO ages derived from the D08 and DM97 evolutionary tracks results in similar $\kappa$ values $\sim-0.24$, an indicator of the accelerating star formation in L1641. The age distribution derived using the S00 tracks indicates that the star formation started $\sim 10$ Myr ago, and increased with time. The star formation rate peaked 2-3 Myr ago. The age distribution derived using the Pisa11 tracks displays an accelerating star formation rate starting $\sim 10 \mathrm{Myr}$ ago, similar to those from other PMS evolutionary tracks. However, the star formation rate has been constant since $3 \mathrm{Myr}$ ago until now. Whether or not the stellar ages from the PMS isochrone are accurate enough to constrain the SFH in star-forming regions is still a matter of debate (Palla \& Stahler 2000; Hartmann 2001; Hillenbrand et al. 2008). Generally, though the age distribution varied when derived with different evolutionary tracks, they showed a common characteristic: in L1641, star formation was very inactive until $5 \mathrm{Myr}$ ago and has been active for the past 2-3 Myr.

Observationally, the lifetimes of the Class I or flat-spectrum stages are statistically estimated using the ratio between the number of Class I and flat-spectrum sources and Class II sources, assuming an age, e.g., $2 \pm 1 \mathrm{Myr}$, for Class II sources, and an SFH (Evans et al. 2009). Thus, the knowledge of the SFH is important in estimating the lifetimes of these sources. In the literature, the SFH of the studied region is usually assumed to be constant (Evans et al. 2009). However, if the true star formation rate of the studied region is accelerating, then the derived lifetimes of the Class I or flat-spectrum sources would be overestimated. To demonstrate this effect, we employ a simple model using the YSOs from L1641 and from Spitzer c2d as examples. We use the function described in Equation (6) to characterize the SFH. Models with $\kappa>0$ correspond to a decelerating star formation rate, whereas $\kappa<0$ corresponds to an accelerating star formation rate and $\kappa=0$ represents a constant star formation rate. In the model, we assume that Class II sources would evolve into Class III at an age of $3 \mathrm{Myr}$. In L1641, $N(\mathrm{I}) / N(\mathrm{II})$ and $N($ Flat $) / N($ II) are 0.27 and 0.25 , respectively, where $N(\mathrm{I}), N(\mathrm{II})$, and $N$ (Flat) are the number of Class I, Class II, and flat-spectrum sources, respectively. In the c2d survey, both fractions are $\sim 0.27$ and $\sim 0.20$, respectively, for five star-forming regions, i.e., Cha II, Lupus, Serpens, Perseus, and Ophiuchus. In Figure 43, we show how the estimated lifetimes of Class I and Flat-spectrum sources change with the 

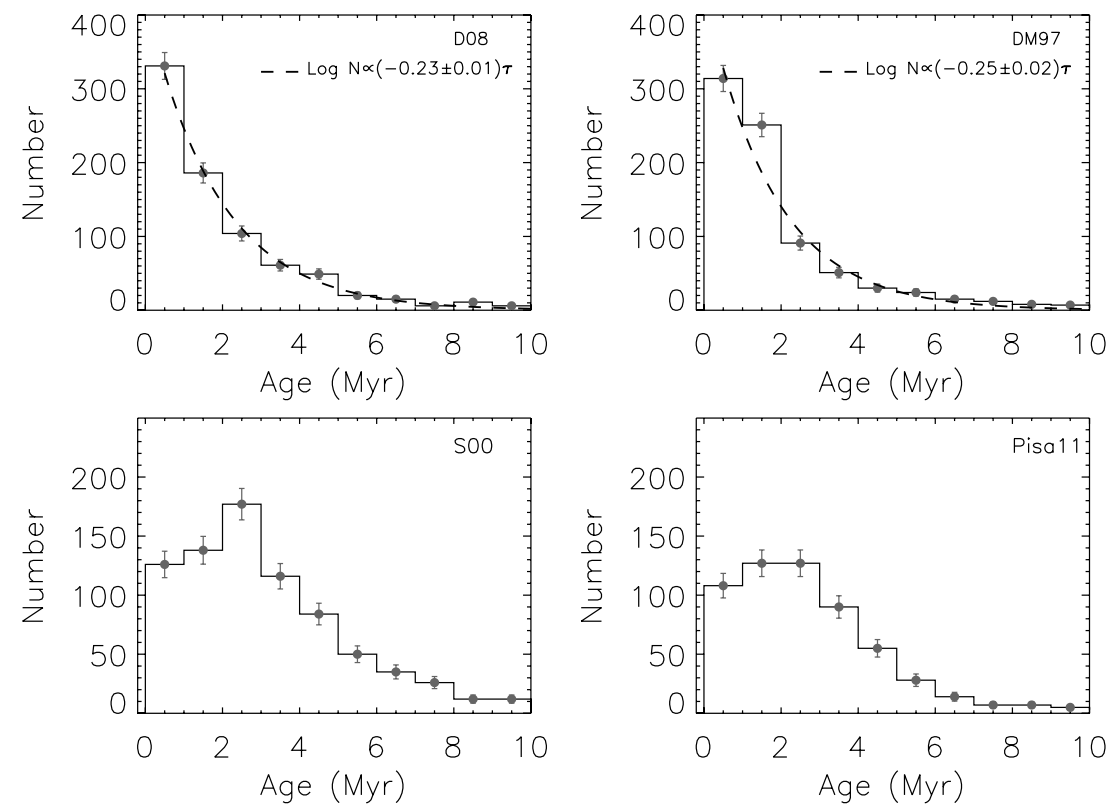

Figure 42. Distribution of ages for the YSOs in our sample derived using different evolutionary tracks: i.e., Dotter et al. (2008, D08), D’Antona \& Mazzitelli (1997, DM97), Siess et al. (2000, S00), and Tognelli et al. (2011, Pisa11).

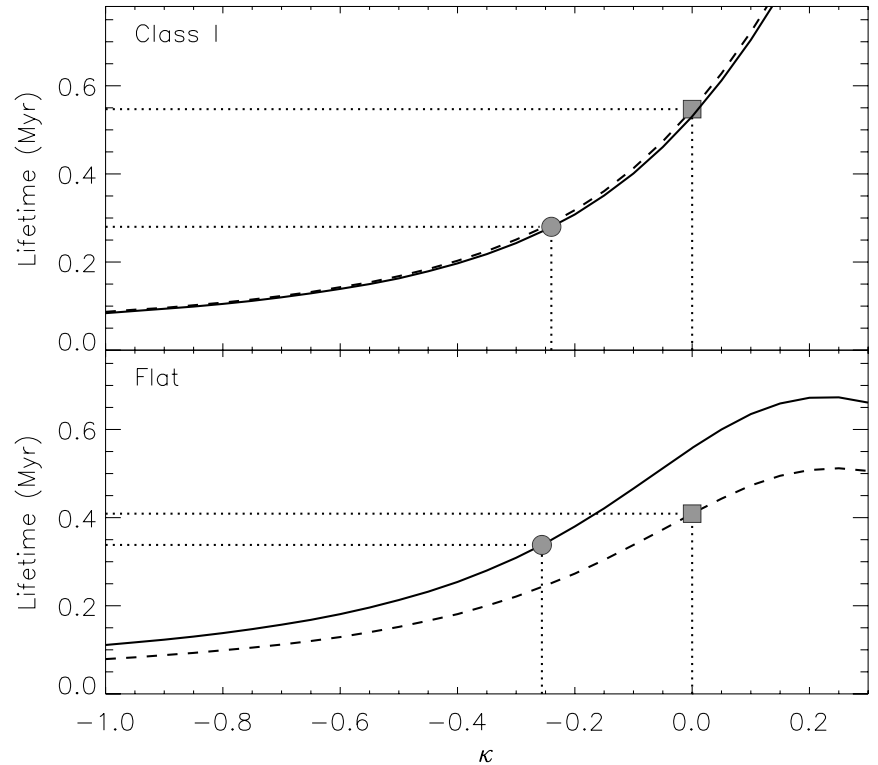

Figure 43. Estimated lifetime of Class I (the upper panel) and flat-spectrum (the lower panel) sources as a function of $\kappa$ values. The solid lines are for YSOs in L1641, and the dashed lines are for YSOs from the Spitzer c2d survey including the Cha II, Lupus, Serpens, Perseus, and Ophiuchus star-forming regions. The parameter $\kappa$ is used to characterize the SFH (see Section 5.4). The squares and circles mark the locations on these curves that correspond to a constant SFH $(\kappa=0)$ or an accelerating SFH that matches our observations $(\kappa=-0.24)$.

different $\kappa$ values. If the star formation rate is assumed to be constant $(\kappa=0)$, then the lifetimes of Class I and Flat-spectrum sources are $\sim 0.54$ and $0.50 \mathrm{Myr}$, respectively, in L1641, and $\sim 0.55$ and $0.41 \mathrm{Myr}$, respectively, for the c $2 \mathrm{~d}$ survey (Evans et al. 2009). However, if $\kappa \sim-0.24$, an accelerating star formation rate, then the lifetimes of both classes would decrease by a factor of two.

\section{SUMMARY}

We have performed a census of YSOs in L1641 using the data from the Spitzer imaging surveys, the $X M M$ surveys, and our spectroscopic surveys. We have spectroscopically surveyed a large sub-sample of YSOs with Hectospec and Hectochelle. We combined the Hectospec data with optical and infrared imaging data to estimate the stellar effective temperatures, luminosities, and extinction values of individual YSOs. The masses and ages of individual YSOs are estimated by their placement in the H-R diagram. Accretion activity is characterized using the $\mathrm{H} \alpha$ and $\mathrm{H} \beta$ lines. The main results in this work are summarized as follows.

1. We presented a catalog of $\sim 1390$ YSOs in L1641 and constructed the SED from 0.4 to $24 \mu \mathrm{m}$ for each YSO. Based on the infrared SEDs, we classified the YSOs into four different classes. We have 143 Class I sources, 131 Flat-spectrum sources, 533 Class II sources, and 507 Class III sources in our YSO catalog.

2. We have used the medium-resolution spectroscopic data from Hectospec in conjunction with the imaging data to derive spectral types, line-of-sight extinction, estimates of the stellar mass/age, EWs of $\mathrm{H} \alpha$ and $\mathrm{H} \beta$ lines, and accretion rates from the $\mathrm{H} \alpha$ and/or $\mathrm{H} \beta$ line luminosities for $\sim 300$ YSOs with spectra from our observations, as well as $\sim 300$ YSOs with spectral types from Hsu et al. (2012).

3. We have analyzed the high-resolution spectroscopic data observed with Hectochelle. For 235 YSOs we are able to examine the $\mathrm{H} \alpha$ emission line profile as well as look for Li I $\lambda 6708$ absorption. In our sample, 65 YSOs have been observed at one epoch, 97 YSOs at two epochs, and 73 at three epochs, allowing us to trace accretion variability.

4. We find one new subluminous object in our spectroscopic sample. It is most likely a star with a nearly edge-on disk in which the cold outer parts of the disk can effectively screen the central star but the $10 \mu \mathrm{m}$ radiation of the warm inner disk can still reach us.

5. Based on our classifications, the disk fraction in L1641, defined as $N(\mathrm{II}) / N(\mathrm{II}+\mathrm{III})$, is $\sim 50 \%$. The disk fraction is almost constant across the stellar mass spectrum with a slight peak at $\log \left(M_{*} / M_{\odot}\right) \approx-0.25$.

6. We find that the ages of young stars with optically thick disks, TDs, and without disks in L1641 all show very broad 
distributions but the median age of diskless YSOs is larger than that of stars with optically thick disks. The median age of stars with TDs is intermediate between these two populations.

7. Based on the infrared spectral slopes, we find that two types of disk dissipation processes, radially depleted and globally depleted evolution, are at work in L1641.

8. The accretion rates of young stars in L1641 are consistent with the $\dot{M}_{\text {acc }} \propto M_{*}^{\alpha}$ relation with $\alpha \sim 2-3$ with a large scatter in $\dot{M}_{\text {acc }}$ for a given stellar mass. We compiled a set of mass and accretion rates in this work and in the literature and found that these data support a scenario proposed by Hartmann et al. (2006) in which the different disk properties around low-mass and brown dwarf stars and intermediatemass stars are responsible for the change in $\alpha$ between intermediate-mass stars and very low mass stars.

9. Based on the multi-epoch data from Hectochelle, we investigated the accretion variability of young stars in L1641 and found typical accretion rate changes of $\sim 0.6$ dex. We confirm that intrinsic accretion variations cannot explain the two orders of magnitude scatter in accretion rates for YSOs with similar masses. We find that the relative variability of $\mathrm{FW}_{\mathrm{H} \alpha, 10 \%}$ for YSOs with disks in the sub-solar mass range is larger than those in the intermediate-mass range.

10. We confirmed 46 new TD objects in L1641, 6 globally depleted disks, and provided 2 young debris disk candidates. We investigated the accretion properties of YSOs with optically thick disks and TDs based on the $\mathrm{H} \alpha$ EWs and $\mathrm{FW}_{\mathrm{H} \alpha, 10 \%}$. We found that the fraction of accretors among TDs (40\%-45\%) is significantly lower than among young stars with optically thick disks (77\%-79\%). Among six globally depleted disks, only one source is accreting. We confirm our previous result that the median accretion rate of accreting TDs is similar to that of accreting stars with optically thick disks. We find that the two types of disks show similar fluctuations in their accretion rates.

11. We estimated the SDs of YSOs in L1641 using the nearest neighbor method. We find that there is no significant difference in the SD distributions between Class III sources, Class II source, and flat-spectrum/Class I sources. We compared the SD distribution of YSOs in L1641 with Taurus and IC 348, and confirmed that both clustered and isolated star formation is ongoing in L1641.

12. We investigated the SFH in L1641 based on $~ 800$ YSOs with spectral-type estimates. We found that the star formation has been most active during the past 2-3 Myr. We also show that knowledge of the SFH is important for constraining the lifetime of Class I and flat-spectrum sources.

We thank Perry Berlind, Mike Calkins, and Nelson Caldwell at SAO for assisting with Hectospec and Hectochelle observations, and the anonymous referee for comments that helped to improve this paper. M.F. acknowledges support by the NSFC through grants 11203081 and 11173060 . A.S.-A. acknowledges support by the Spanish "Ramon y Cajal" program. This research has made use of the SIMBAD database, operated at CDS, Strasbourg, France. This publication makes use of data products from the Two Micron All Sky Survey, which is a joint project of the University of Massachusetts and the Infrared Processing and Analysis Center/California Institute of Technology, funded by the National Aeronautics and Space Administration and the National Science Foundation. This work is based in part on observations made with the Spitzer Space Telescope, which is operated by the Jet Propulsion Laboratory, California Institute of Technology, under a contract with NASA. This publication makes use of data products from the Wide-field Infrared Survey Explorer, which is a joint project of the University of California, Los Angeles, and the Jet Propulsion Laboratory/California Institute of Technology, funded by the National Aeronautics and Space Administration. This work is based in part on data obtained as part of the UKIRT Infrared Deep Sky Survey. This publication is based in part on data from the Sloan Digital Sky Survey project, whose many contributors can be found at the following Web site: http://www.sdss.org/collaboration/credits.html. This research is partially based on observations made with the NASA/ESA Hubble Space Telescope, and obtained from the Hubble Legacy Archive, which is a collaboration between the Space Telescope Science Institute (STScI/NASA), the Space Telescope European Coordinating Facility (ST-ECF/ESA), and the Canadian Astronomy Data Centre (CADC/NRC/CSA).

\section{APPENDIX A}

\section{ACCRETION CHARACTERIZED BY $\mathrm{FW}_{\mathrm{H} \alpha, 10 \%}$}

White \& Basri (2003) proposed using the $\mathrm{FW}_{\mathrm{H} \alpha, 10 \%}$ instead of the $\mathrm{EW}$ of the $\mathrm{H} \alpha$ line as an alternative to distinguishing accretors from non-accretors. They found that young stars with $\mathrm{FW}_{\mathrm{H} \alpha, 10 \%}$ greater than $270 \mathrm{~km} \mathrm{~s}^{-1}$ are accretors. Since a large sample of young stars with or without disks that have been observed with high-resolution $\mathrm{H} \alpha$ spectroscopy exists in the literature, we can statistically study how to use $\mathrm{FW}_{\mathrm{H} \alpha, 10 \%}$ to classify CTTSs and WTTSs.

Our basic method is described as follows. We select from the literature young stars that show no evidence for significant infrared excess at $\lambda<8 \mu \mathrm{m}$, i.e., their (inner) disks have already dissipated. The vast majority of these stars will not be actively accreting, though the sample can contain a few TDs, some of which may still accrete. We then plot the frequency distribution of $\mathrm{FW}_{\mathrm{H} \alpha, 10 \%}$ for all of these objects and use a function to fit the distribution. We normalize the fitted function and use it as the probability function of $\mathrm{FW}_{\mathrm{H} \alpha, 10 \%}$ for non-accreting young stars.

We collected young stars from the literature covering many different star formation regions, i.e., the Orion nebular cluster (Fûrész et al. 2008; Tobin et al. 2009), NGC 2068/2071 (Flaherty \& Muzerolle 2008), Tr37 (Sicilia-Aguilar et al. 2006b, 2006a), $\eta$ Cha, TW Hydrae, $\beta$ Pictoris, and Tucanae-Horologium groups (Sicilia-Aguilar et al. 2009; Jayawardhana et al. 2006), Cha I, and Taurus-Auriga star-forming regions (Nguyen et al. 2009a). In total, 334 stars satisfy our criteria for having no or weak infrared excess $(|[3.6]-[4.5]| \leqslant 0.2$ and $|[5.8]-[8.0]| \leqslant$ $0.2)$. The inner disks around these stars have been mostly cleared away and the majority of these targets should no longer be actively accreting. In Figure 44, we show the [5.8] - [8.0] versus [3.6] - [4.5] color-color diagram for these targets and the fractional distribution of $\mathrm{FW}_{\mathrm{H} \alpha, 10 \%}$. As shown in Figure 44(b), the distribution of $\mathrm{FW}_{\mathrm{H} \alpha, 10 \%}$ for "diskless" YSOs has a sharp peak with a long tail toward large $\mathrm{FW}_{\mathrm{H} \alpha, 10 \%}$. The long tail could be due to accreting TDs since we only use Spitzer IRAC bands to select the "diskless" YSOs and disks with large inner holes cannot be distinguished from diskless YSOs without data at longer wavelengths. We fit the distribution with two Gaussian functions and normalize the fitted function to a total area of 1 . 

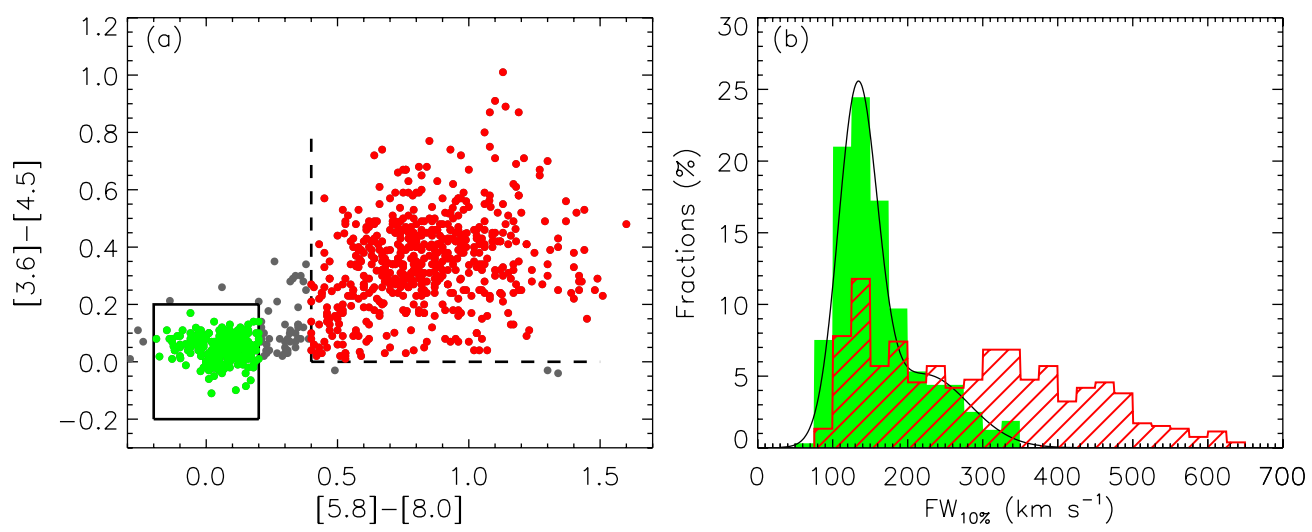

Figure 44. (a) [3.6] - [4.5] vs. [5.8] - [8.0] color-color diagram for young stars with high-resolution $\mathrm{H} \alpha$ spectroscopy. The solid lines enclose the region containing diskless stars. We use the $\mathrm{FW}_{\mathrm{H} \alpha, 10 \%}$ of these diskless stars to derive the accretion probability as a function of $\mathrm{FW}_{\mathrm{H} \alpha, 10 \%}$. The dashed lines mark the region containing Class II YSOs. (b) The distribution of $\mathrm{FW}_{\mathrm{H} \alpha, 10 \%}$ for diskless stars (filled histogram) and Class II YSOs (line-filled histogram).

(A color version of this figure is available in the online journal.)

The normalized function is

$$
\begin{aligned}
& \Phi\left(\mathrm{FW}_{\mathrm{H} \alpha, 10 \%}\right)=1.04 \times 10^{-2} \exp \left[-\left(\frac{\mathrm{FW}_{\mathrm{H} \alpha, 10 \%}-133.23}{36.96}\right)^{2}\right] \\
& +2.20 \times 10^{-3} \exp \left[-\left(\frac{\mathrm{FW}_{\mathrm{H} \alpha, 10 \%}-223.04}{82.35}\right)^{2}\right] .
\end{aligned}
$$

We use the above function as the probability function of $\mathrm{FW}_{\mathrm{H} \alpha, 10 \%}$ for non-accretors. Thus, for a YSO with an estimate of $\mathrm{FW}_{\mathrm{H} \alpha, 10 \%}$, the probability that it is a non-accretor is estimated to be $\int_{\mathrm{FW}_{\mathrm{H} \alpha, 10 \%}^{\infty}}^{\infty} \Phi(x) d x$. The probability that it is an accretor is $\operatorname{prob}\left(\mathrm{FW}_{\mathrm{H} \alpha, 10 \%}\right)=1-\int_{\mathrm{FW}_{\mathrm{H} \alpha} 10 \%}^{\infty} \Phi(x) d x$. A source with $\mathrm{FW}_{\mathrm{H} \alpha, 10 \%}>250 \mathrm{~km} \mathrm{~s}^{-1}$ has a probability of $90 \%$ of being an accretor. Thus, we use $\mathrm{FW}_{\mathrm{H} \alpha, 10 \%}>250 \mathrm{~km} \mathrm{~s}^{-1}$ as our criterion for distinguishing accretors from non-accretors. According to this criterion, $56 \%$ of the Class II sources shown in Figure 44 are accretors.

\section{APPENDIX B}

\section{COMPARISON OF ACCRETION RATES FROM $\mathrm{H} \alpha$ LINE LUMINOSITY AND $\mathrm{FW}_{\mathrm{H} \alpha, 10 \%}$}

In our sample of young stars, there are $\sim 90$ accreting sources that have been observed with Hectochelle. Using these data, we estimate the accretion rates from both $\mathrm{FW}_{\mathrm{H} \alpha, 10 \%}$ and $\mathrm{H} \alpha$ line luminosity as described in Section 3.4.2. We compare the accretion rates derived using the two methods in Figure 45. In order to see whether there is any correlation between both types of accretion rates, we apply a Kendall $\tau$ test. If two data sets are fully correlated, then this test returns a value of $\tau=1$. If they are anti-correlated, then we get $\tau=-1$, and if they are independent, we obtain $\tau=0$. The Kendall $\tau$ test also returns a probability $p$, which is smaller when the correlation is more significant. We use the Kendall $\tau$ test to evaluate the possible correlation between the accretion rates from the two methods, which yields $\tau=0.3$ and $p=1.5 \times 10^{-5}$. Thus, though the scatter is large in Figure 45, the correlation between two kinds of accretion rates is existent. We also note that the accretion rates from $\mathrm{FW}_{\mathrm{H} \alpha 10 \%}$ are systematically lower than those from $\mathrm{H} \alpha$ line luminosity. It is beyond the scope of the current work to distinguish the better tracer for accretion and calibrate the accretion tracer to the accretion rates. A series of works using

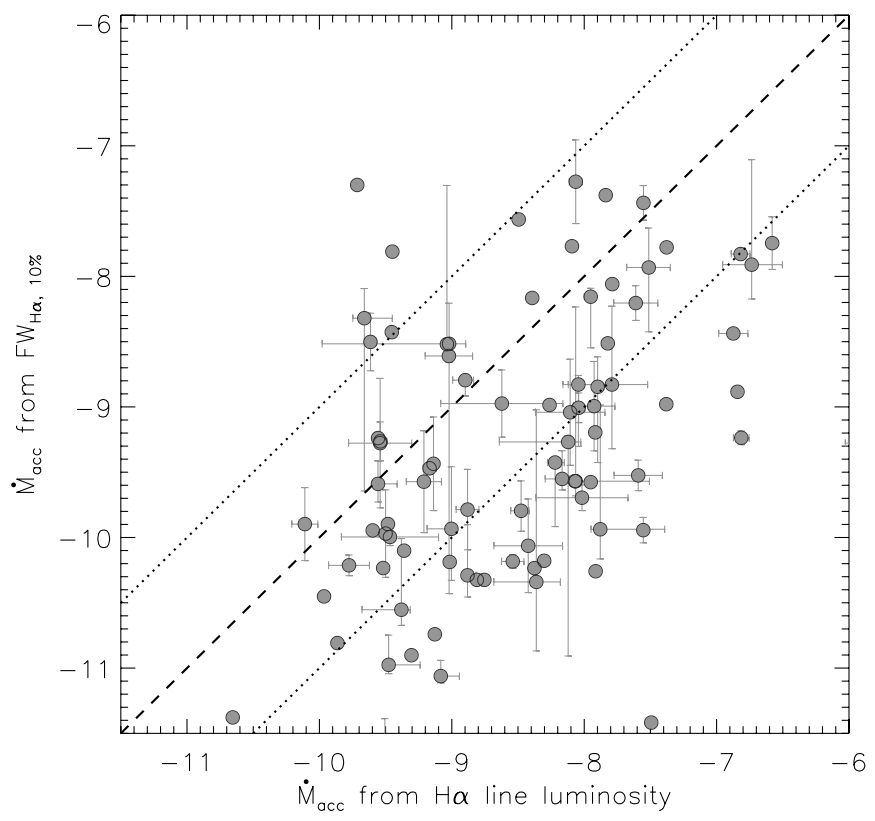

Figure 45. Comparison of accretion rates of YSOs in L1641 derived from $\mathrm{FW}_{\mathrm{H} \alpha, 10 \%}$ and $\mathrm{H} \alpha$ line luminosity. For the sources with multiple observations, the median accretion rates are used for the plot with error bars showing their minimum and maximum accretion rates. The dashed line shows a 1:1 correlation, and dotted lines show a difference of 1 dex.

the data from VLT/X-shooter (see, e.g., Rigliaco et al. 2011b, 2012) has been performed or is ongoing.

\section{APPENDIX C}

\section{EVALUATING COMPLETENESS OF YSO CENSUS USING COLOR-MAGNITUDE DIAGRAM}

In Figure 46(a), we show the $r$ versus $r-i$ color-magnitude diagram for $\sim 660$ YSOs in L1641 with photometric data in both $r$ and $i$ bands. Among these sources, more than $70 \%$ of them are located within a narrow band, in the $r$ versus $r-i$ color-magnitude diagram. We can use the locations of the known YSOs to select the potential YSO candidates. However, the YSO candidates selected in this way could be contaminated by the reddened field stars. The fractions of contaminators can be estimated if we know how the field stars are distributed in the $r$ versus $r-i$ color-magnitude diagram. We create 10 sets 

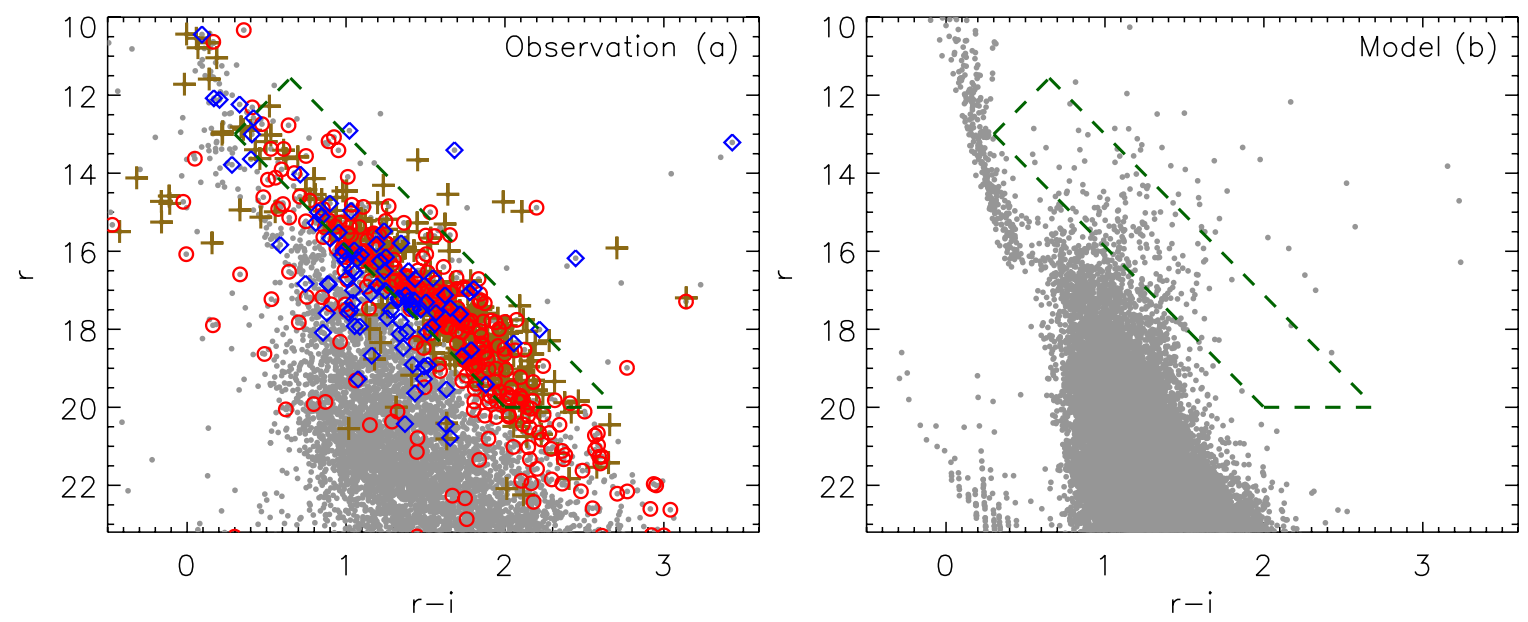

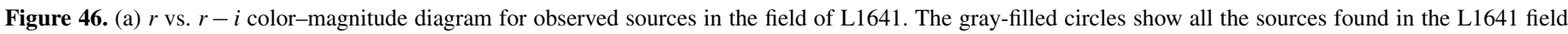

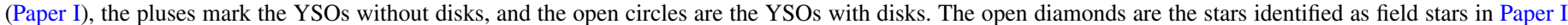

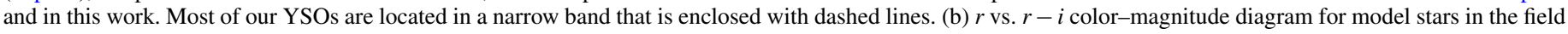
of L1641, which are created with the Besançon model of stellar population synthesis of the Galaxy (Robin et al. 2003).

(A color version of this figure is available in the online journal.)

of model stars in the direction of L1641 over the same area we studied using the Besançon model of stellar population synthesis of the Galaxy (Robin et al. 2003). We derive the extinction map of the L1641 cloud from the ${ }^{13} \mathrm{CO}$ map (see Figure 2) using the relation between the ${ }^{13} \mathrm{CO}$ intensity and visual extinction from Paper I. An extinction probability function is built from the extinction map of the L1641 cloud. For each model star with a distance $>500 \mathrm{pc}$, an extinction value, randomly sampled from the extinction probability function, is assigned to it to simulate the reddening effect on the background stars from the L1641 cloud. For each set of model stars from the Besançon model, we sample 100 groups of extinction values from the extinction probability function. In total, we obtain 1000 model $r$ versus $r-i$ color-magnitude diagrams for 10 sets of model stars. In Figure 46(b), we show the $r$ versus $r-i$ color-magnitude diagram for one set of model stars. The Besançon model predicts that the field stars contaminate our YSO sample mostly within $1.0 \lesssim r-i \lesssim 1.5$ and $14.0 \lesssim r \lesssim 17.5$ in the $r$ versus $r-i$ color-magnitude diagram, which is generally consistent with our observations (see Figures 46(a) and (b)).

We visually define a region in the $r$ versus $r-i$ color-magnitude diagram (enclosed by dashed lines in Figure 46) where most of these YSOs are located. We study only the sources with $r<20$ mag, considering that the completeness of the imaging survey in the $r$ band is around 20 mag. Within our selected region, 190 sources are not included in our YSO sample. Among them, 39 sources have been identified as field stars in Paper I and this work. With the synthetic models, $194 \pm 12$ field stars are predicted to be within our defined region in the $r$ versus $r-i$ color-magnitude diagram, suggesting that our YSO census is complete till $r \sim 20 \mathrm{mag}$.

\section{REFERENCES}

Alencar, S. H. P., \& Batalha, C. 2002, ApJ, 571, 378

Alencar, S. H. P., Johns-Krull, C. M., \& Basri, G. 2001, AJ, 122, 3335

Alexander, R. D., \& Armitage, P. J. 2009, ApJ, 704, 989

Alexander, R. D., Clarke, C. J., \& Pringle, J. E. 2006, MNRAS, 369, 229

Allen, L. E. 1995, PhD thesis, Univ. Massachusetts, Amherst

Allen, T. S., Gutermuth, R. A., Kryukova, E., et al. 2012, ApJ, 750, 125

Alves, J., \& Bouy, H. 2012, A\&A, 547, A97

Andrews, S. M., \& Williams, J. P. 2005, ApJ, 631, 1134

Anthony-Twarog, B. J. 1982, AJ, 87, 1213
Armitage, P. J., Clarke, C. J., \& Palla, F. 2003, MNRAS, 342, 1139

Balbus, S. A., \& Hawley, J. F. 1998, RvMP, 70, 1

Bally, J., Stark, A. A., Wilson, R. W., \& Langer, W. D. 1987, ApJL, 312, L45

Baraffe, I., Chabrier, G., Allard, F., \& Hauschildt, P. H. 1998, A\&A, 337, 403

Baraffe, I., Chabrier, G., Allard, F., \& Hauschildt, P. H. 2002, A\&A, 382, 563

Bertout, C., Siess, L., \& Cabrit, S. 2007, A\&A, 473, L21

Bessell, M. S., \& Brett, J. M. 1988, PASP, 100, 1134

Bouwman, J., Lawson, W. A., Dominik, C., et al. 2006, ApJL, 653, L57

Brandner, W., Grebel, E. K., Chu, Y.-H., et al. 2000, AJ, 119, 292

Calvet, N., Muzerolle, J., Briceño, C., et al. 2004, AJ, 128, 1294

Caratti O Garatti, A., Garcia Lopez, R., Antoniucci, S., et al. 2012, A\&A, 538, A64

Cardelli, J. A., Clayton, G. C., \& Mathis, J. S. 1989, ApJ, 345, 245

Carpenter, J. M., Bouwman, J., Mamajek, E. E., et al. 2009, ApJS, 181, 197

Carpenter, J. M., Wolf, S., Schreyer, K., Launhardt, R., \& Henning, T. 2005, AJ, 129,1049

Carrera, F. J., Ebrero, J., Mateos, S., et al. 2007, A\&A, 469, 27

Chen, H., \& Tokunaga, A. T. 1994, ApJS, 90, 149

Chiang, E., \& Murray-Clay, R. 2007, NatPh, 3, 604

Cieza, L. A., Schreiber, M. R., Romero, G. A., et al. 2010, ApJ, 712, 925

Clarke, C. J., Gendrin, A., \& Sotomayor, M. 2001, MNRAS, 328, 485

Comerón, F., Fernández, M., Baraffe, I., Neuhäuser, R., \& Kaas, A. A. 2003, A\&A, 406, 1001

Connelley, M. S., \& Greene, T. P. 2010, AJ, 140, 1214

Currie, T. 2010, arXiv:1002.1715

Currie, T., \& Kenyon, S. J. 2009, AJ, 138, 703

Currie, T., Lada, C. J., Plavchan, P., et al. 2009, ApJ, 698, 1

Currie, T., \& Sicilia-Aguilar, A. 2011, ApJ, 732, 24

Dahm, S. E. 2008, AJ, 136, 521

Dahm, S. E., \& Hillenbrand, L. A. 2007, AJ, 133, 2072

Dahm, S. E., \& Simon, T. 2005, AJ, 129, 829

D’Antona, F., \& Mazzitelli, I. 1997, MmSAI, 68, 807

Dotter, A., Chaboyer, B., Jevremović, D., et al. 2008, ApJS, 178, 89

Dullemond, C. P., Natta, A., \& Testi, L. 2006, ApJL, 645, L69

Espaillat, C., Ingleby, L., Hernández, J., et al. 2012, ApJ, 747, 103

Evans, N. J., Dunham, M. M., Jørgensen, J. K., et al. 2009, ApJS, 181, 321

Fabricant, D., Fata, R., Roll, J., et al. 2005, PASP, 117, 1411

Fang, M., van Boekel, R., Bouwman, J., et al. 2013, A\&A, 549, A15

Fang, M., van Boekel, R., King, R. R., et al. 2012, A\&A, 539, A119

Fang, M., van Boekel, R., Wang, W., et al. 2009, A\&A, 504, 461 (Paper I)

Feigelson, E. D., Gaffney, J. A., III, Garmire, G., Hillenbrand, L. A., \& Townsley, L. 2003, ApJ, 584, 911

Feigelson, E. D., \& Montmerle, T. 1999, ARA\&A, 37, 363

Fischer, D. A., \& Marcy, G. W. 1992, ApJ, 396, 178

Flaherty, K. M., \& Muzerolle, J. 2008, AJ, 135, 966

Flaherty, K. M., Pipher, J. L., Megeath, S. T., et al. 2007, ApJ, 663, 1069

Fúrész, G., Hartmann, L. W., Megeath, S. T., Szentgyorgyi, A. H., \& Hamden, E. T. $2008, \mathrm{ApJ}, 676,1109$

Furlan, E., Hartmann, L., Calvet, N., et al. 2006, ApJS, 165, 568

Gâlfalk, M., \& Olofsson, G. 2008, A\&A, 489, 1409 
Gammie, C. F. 1996, ApJ, 457, 355

Garcia Lopez, R., Natta, A., Testi, L., \& Habart, E. 2006, A\&A, 459, 837 Gatti, T., Natta, A., Randich, S., Testi, L., \& Sacco, G. 2008, A\&A, 481, 423

Genzel, R., Reid, M. J., Moran, J. M., \& Downes, D. 1981, ApJ, 244, 884

Ghez, A. M., McCarthy, D. W., Patience, J. L., \& Beck, T. L. 1997, ApJ, 481,378

Ghez, A. M., Neugebauer, G., \& Matthews, K. 1993, AJ, 106, 2005

Glassgold, A. E., Najita, J., \& Igea, J. 1997, ApJ, 480, 344

Guarcello, M. G., Prisinzano, L., Micela, G., et al. 2007, A\&A, 462, 245

Güdel, M. 2004, A\&ARv, 12, 71

Güdel, M., Briggs, K. R., Arzner, K., et al. 2007, A\&A, 468, 353

Gullbring, E., Hartmann, L., Briceno, C., \& Calvet, N. 1998, ApJ, 492, 323

Gullbring, E., Petrov, P. P., Ilyin, I., et al. 1996, A\&A, 314, 835

Gustafsson, B., Edvardsson, B., Eriksson, K., et al. 2008, A\&A, 486, 951

Gutermuth, R. A., Myers, P. C., Megeath, S. T., et al. 2008, ApJ, 674, 336

Haisch, K. E., Jr., Lada, E. A., \& Lada, C. J. 2001, ApJL, 553, L153

Hartigan, P., Edwards, S., \& Ghandour, L. 1995, ApJ, 452, 736

Hartigan, P., \& Kenyon, S. J. 2003, ApJ, 583, 334

Hartmann, L. 1999, NewAR, 43, 1

Hartmann, L. 2001, AJ, 121, 1030

Hartmann, L., Calvet, N., Gullbring, E., \& D’Alessio, P. 1998, ApJ, 495, 385

Hartmann, L., D’Alessio, P., Calvet, N., \& Muzerolle, J. 2006, ApJ, 648, 484

Hartmann, L., Hewett, R., \& Calvet, N. 1994, ApJ, 426, 669

Hayashi, C., Nakazawa, K., \& Nakagawa, Y. 1985, in Protostars and Planets II, ed. D. C. Black \& M. S. Matthews (Tucson, AZ: Univ. Arizona Press), 1100

Herbig, G. H. 1998, ApJ, 497, 736

Herbig, G. H., \& Dahm, S. E. 2002, AJ, 123, 304

Herczeg, G. J., \& Hillenbrand, L. A. 2008, ApJ, 681, 594

Hernández, J., Calvet, N., Briceño, C., Hartmann, L., \& Berlind, P. 2004, AJ, 127,1682

Hernández, J., Calvet, N., Briceño, C., et al. 2007a, ApJ, 671, 1784

Hernández, J., Hartmann, L., Megeath, T., et al. 2007b, ApJ, 662, 1067

Hernández, J., Morales-Calderon, M., Calvet, N., et al. 2010, ApJ, 722, 1226

Hillenbrand, L. A. 1997, AJ, 113, 1733

Hillenbrand, L. A. 2002, arXiv:astro-ph/0210520

Hillenbrand, L. A., Bauermeister, A., \& White, R. J. 2008, in ASP Conf. Ser. 384, 14th Cambridge Workshop on Cool Stars, Stellar Systems, and the Sun, ed. G. van Belle (San Francisco, CA: ASP), 200

Hillenbrand, L. A., \& White, R. J. 2004, ApJ, 604, 741

Hirota, T., Bushimata, T., Choi, Y. K., et al. 2007, PASJ, 59, 897

Hollenbach, D. J., Yorke, H. W., \& Johnstone, D. 2000, in Protostars and Planets IV, ed. V. Mannings, A. P. Boss, \& S. S. Russell (Tucson, AZ: Univ. Arizona Press), 401

Hsu, W.-H., Hartmann, L., Allen, L., et al. 2012, ApJ, 752, 59

Hsu, W.-H., Hartmann, L., Allen, L., et al. 2013, ApJ, 764, 114

Jayawardhana, R., Coffey, J., Scholz, A., Brandeker, A., \& van Kerkwijk, M. H. 2006, ApJ, 648, 1206

Jeffries, R. D., Jackson, R. J., James, D. J., \& Cargile, P. A. 2009, MNRAS, 400,317

Johns, C. M., \& Basri, G. 1995, ApJ, 449, 341

Johnstone, D., Hollenbach, D., \& Bally, J. 1998, ApJ, 499, 758

Kenyon, S. J., \& Hartmann, L. 1995, ApJS, 101, 117

Kurosawa, R., Romanova, M. M., \& Harries, T. J. 2011, MNRAS, 416, 2623

Kurucz, R. 1994, Solar Abundance Model Atmospheres for 0, 1, 2, 4, $8 \mathrm{~km} \mathrm{~s}^{-1}$ (Kurucz CD-ROM No. 19; Cambridge, MA: Smithsonian Astrophysical Observatory), 19

Kurucz, R. L. 1979, ApJS, 40, 1

Lada, C. J., Muench, A. A., Luhman, K. L., et al. 2006, AJ, 131, 1574

Lada, E. A., \& Lada, C. J. 1995, AJ, 109, 1682

Lafrenière, D., Jayawardhana, R., Brandeker, A., Ahmic, M., \& van Kerkwijk, M. H. 2008, ApJ, 683, 844

Lawrence, A., Warren, S. J., Almaini, O., et al. 2007, MNRAS, 379, 1599

Leinert, C., Zinnecker, H., Weitzel, N., et al. 1993, A\&A, 278, 129

Lima, G. H. R. A., Alencar, S. H. P., Calvet, N., Hartmann, L., \& Muzerolle, J. 2010, A\&A, 522, A104

Lin, D. N. C., \& Papaloizou, J. C. B. 1993, in Protostars and Planets III, ed. E. H. Levy \& J. I. Lunine (Tucson, AZ: Univ. Arizona Press), 749

Luhman, K. L., Allen, L. E., Allen, P. R., et al. 2008, ApJ, 675, 1375

Luhman, K. L., Allen, P. R., Espaillat, C., Hartmann, L., \& Calvet, N. 2010, ApJS, 186, 111

Luhman, K. L., Stauffer, J. R., Muench, A. A., et al. 2003, ApJ, 593, 1093

Maddalena, R. J., Morris, M., Moscowitz, J., \& Thaddeus, P. 1986, ApJ, 303,375

Mamajek, E. E., Meyer, M. R., Hinz, P. M., et al. 2004, ApJ, 612, 496

Mayne, N. J., Harries, T. J., Rowe, J., \& Acreman, D. M. 2012, MNRAS, 423, 1775

Megeath, S. T., Gutermuth, R., Muzerolle, J., et al. 2012, AJ, 144, 192
Mendigutía, I., Eiroa, C., Montesinos, B., et al. 2011, A\&A, 529, A34

Menten, K. M., Reid, M. J., Forbrich, J., \& Brunthaler, A. 2007, A\&A, 474, 515

Mentuch, E., Brandeker, A., van Kerkwijk, M. H., Jayawardhana, R., \& Hauschildt, P. H. 2008, ApJ, 689, 1127

Merín, B., Brown, J. M., Oliveira, I., et al. 2010, ApJ, 718, 1200

Meyer, M. R., \& Beckwith, S. V. W. 2000, in ISO Survey of a Dusty Universe, ed. D. Lemke, M. Stickel, \& K. Wilke (Lecture Notes in Physics, Vol. 548; Berlin: Springer), 341

Meyer, M. R., Calvet, N., \& Hillenbrand, L. A. 1997, AJ, 114, 288

Mohanty, S., Jayawardhana, R., \& Basri, G. 2005, ApJ, 626, 498

Mottram, J. C., Vink, J. S., Oudmaijer, R. D., \& Patel, M. 2007, MNRAS, 377,1363

Muench, A. A., Lada, C. J., Luhman, K. L., Muzerolle, J., \& Young, E. 2007, AJ, 134,411

Muench, A. A., Lada, E. A., \& Lada, C. J. 2000, ApJ, 533, 358

Muench, A. A., Lada, E. A., Lada, C. J., \& Alves, J. 2002, ApJ, 573, 366

Muench, A. A., Lada, E. A., Lada, C. J., et al. 2003, AJ, 125, 2029

Muzerolle, J., Allen, L. E., Megeath, S. T., Hernández, J., \& Gutermuth, R. A. 2010, ApJ, 708, 1107

Muzerolle, J., Calvet, N., \& Hartmann, L. 2001, ApJ, 550, 944

Muzerolle, J., Hartmann, L., \& Calvet, N. 1998, AJ, 116, 2965

Muzerolle, J., Hillenbrand, L., Calvet, N., Briceño, C., \& Hartmann, L. 2003, ApJ, 592, 266

Muzerolle, J., Luhman, K. L., Briceño, C., Hartmann, L., \& Calvet, N 2005, ApJ, 625, 906

Najita, J. R., Strom, S. E., \& Muzerolle, J. 2007, MNRAS, 378, 369

Natta, A., Testi, L., Muzerolle, J., et al. 2004, A\&A, 424, 603

Natta, A., Testi, L., \& Randich, S. 2006, A\&A, 452, 245

Nguyen, D. C., Jayawardhana, R., van Kerkwijk, M. H., et al. 2009a, ApJ, 695,1648

Nguyen, D. C., Scholz, A., van Kerkwijk, M. H., Jayawardhana, R., \& Brandeker, A. 2009b, ApJL, 694, L153

O'dell, C. R., Wen, Z., \& Hu, X. 1993, ApJ, 410, 696

Osterloh, M., \& Beckwith, S. V. W. 1995, ApJ, 439, 288

Palla, F., \& Stahler, S. W. 2000, ApJ, 540, 255

Pontoppidan, K. M., Dullemond, C. P., van Dishoeck, E. F., et al. 2005, ApJ, 622,463

Pott, J.-U., Perrin, M. D., Furlan, E., et al. 2010, ApJ, 710, 265

Quillen, A. C., Blackman, E. G., Frank, A., \& Varnière, P. 2004, ApJL, 612, L137

Reipurth, B., Pedrosa, A., \& Lago, M. T. V. T. 1996, A\&AS, 120, 229

Rice, W. K. M., Wood, K., Armitage, P. J., Whitney, B. A., \& Bjorkman, J. E. 2003, MNRAS, 342, 79

Richling, S., \& Yorke, H. W. 2000, ApJ, 539, 258

Rieke, G. H., \& Lebofsky, M. J. 1985, ApJ, 288, 618

Rigliaco, E., Natta, A., Randich, S., Testi, L., \& Biazzo, K. 2011a, A\&A, 525, A47

Rigliaco, E., Natta, A., Randich, S., et al. 2011b, A\&A, 526, L6

Rigliaco, E., Natta, A., Testi, L., et al. 2012, A\&A, 548, A56

Robin, A. C., Reylé, C., Derrière, S., \& Picaud, S. 2003, A\&A, 409, 523

Robitaille, T. P., Whitney, B. A., Indebetouw, R., \& Wood, K. 2007, ApJS, 169,328

Sestito, P., Palla, F., \& Randich, S. 2008, A\&A, 487, 965

Sicilia-Aguilar, A., Bouwman, J., Juhász, A., et al. 2009, ApJ, 701, 1188

Sicilia-Aguilar, A., Hartmann, L., Calvet, N., et al. 2006a, ApJ, 638, 897

Sicilia-Aguilar, A., Hartmann, L. W., Fürész, G., et al. 2006b, AJ, 132, 2135

Sicilia-Aguilar, A., Henning, T., Dullemond, C. P., et al. 2011, ApJ, 742, 39

Sicilia-Aguilar, A., Henning, T., \& Hartmann, L. W. 2010, ApJ, 710, 597

Sicilia-Aguilar, A., Henning, T., Juhász, A., et al. 2008, ApJ, 687, 1145

Sicilia-Aguilar, A., Henning, T., Linz, H., et al. 2013, A\&A, 551, A34

Siess, L., Dufour, E., \& Forestini, M. 2000, A\&A, 358, 593

Skrutskie, M. F., Cutri, R. M., Stiening, R., et al. 2006, AJ, 131, 1163

Störzer, H., \& Hollenbach, D. 1999, ApJ, 515, 669

Strom, K. M., Newton, G., Strom, S. E., et al. 1989a, ApJS, 71, 183

Strom, K. M., Strom, S. E., Edwards, S., Cabrit, S., \& Skrutskie, M. F. 1989b, AJ, 97,1451

Strom, K. M., Strom, S. E., \& Merrill, K. M. 1993, ApJ, 412, 233

Szentgyorgyi, A., Furesz, G., Cheimets, P., et al. 2011, PASP, 123, 1188

Tobin, J. J., Hartmann, L., Furesz, G., Mateo, M., \& Megeath, S. T. 2009, ApJ, 697, 1103

Tognelli, E., Prada Moroni, P. G., \& Degl'Innocenti, S. 2011, A\&A, 533, A109

Vink, J. S., Drew, J. E., Harries, T. J., \& Oudmaijer, R. D. 2002, MNRAS, 337,356

Watson, M. G., Schröder, A. C., Fyfe, D., et al. 2009, A\&A, 493, 339 
White, R. J., \& Basri, G. 2003, ApJ, 582, 1109

White, R. J., \& Hillenbrand, L. A. 2004, ApJ, 616, 998

Wilson, B. A., Dame, T. M., Masheder, M. R. W., \& Thaddeus, P. 2005, A\&A, 430, 523

Winston, E., Megeath, S. T., Wolk, S. J., et al. 2009, AJ, 137, 4777

Winston, E., Megeath, S. T., Wolk, S. J., et al. 2010, AJ, 140, 266
Wolk, S. J. 2009, in AIP Conf. Proc. 1094, Cool Stars, Stellar Systems and the Sun, ed. E. Stempels (Melville, NY: AIP), 959

Wright, E. L., Eisenhardt, P. R. M., Mainzer, A. K., et al. 2010, AJ, 140,1868

York, D. G., Adelman, J., Anderson, J. E., Jr., et al. 2000, AJ, 120, 1579

Zhang, M., \& Wang, H. 2009, AJ, 138, 1830 